Aus der Abteilung Anaesthesiologie

(Prof. Dr. med. M. Quintel)

im Zentrum Anaesthesiologie, Rettungs- und Intensivmedizin

der Medizinischen Fakultät der Universität Göttingen

\title{
Untersuchungen zum Atemwegsmanagement bei präklinischen Kindernotfällen
}

\author{
INAUGURAL - DISSERTATION \\ zur Erlangung des Doktorgrades \\ der Medizinischen Fakultät \\ der Georg-August-Universität zu Göttingen
}

vorgelegt von

Marcus Nemeth

aus

Hann. Münden 
Dekan:

Prof. Dr. med. C. Frömmel

I. Berichterstatter: $\quad$ Prof. Dr. med. M. Quintel

II. Berichterstatter: $\quad$ Priv.-Doz. Dr. med. M. Sigler 


\section{Inhaltsverzeichnis}

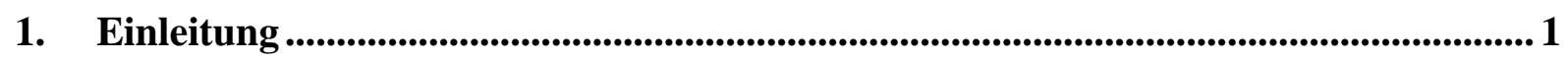

1.1. Die Bedeutung der Atemwegssicherung bei Kindernotfällen ................................. 1

1.2. Besonderheiten des kindlichen Atemweges ............................................................... 3

1.3. Die präklinische Intubation von Kindern ......................................................... 5

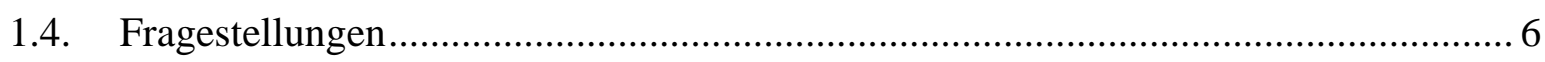

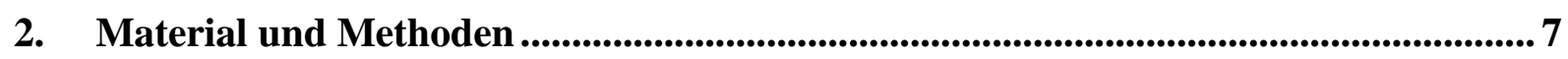

2.1. Studiendesign und Patientenkollektiv ............................................................. 7

2.2. Struktur und Organisation des Notarztdienstes in Göttingen ................................... 8

2.2.1. Pädiatrische Notfallversorgung in der Stadt und Region Göttingen .................... 8

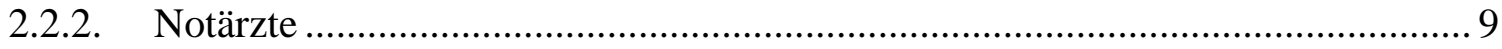

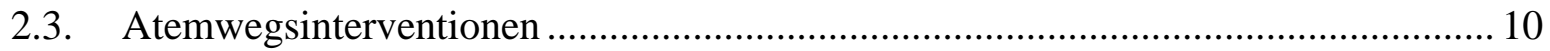

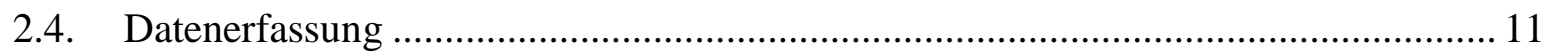

2.4.1. Erfassung der Atemwegscharakteristika ................................................... 12

2.4.2. Schweregrad- und Outcome-Erfassung mittels Scoring-Systemen ................... 13

2.4.2.1. Cormack-Lehane-Score _...................................................................... 13

2.4.2.2. National Advisory Committee on Aeronautics-Index (NACA) ................. 14

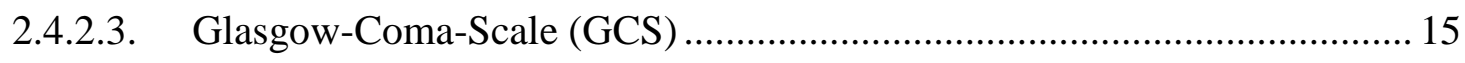

2.4.2.4. Paediatric Cerebral Performance Category (PCPC) …............................ 17

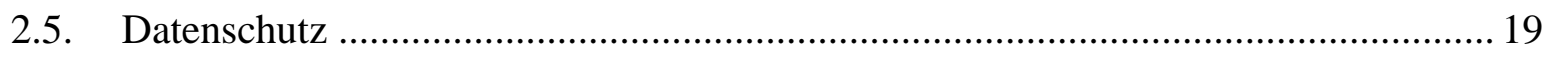

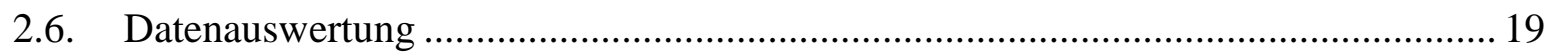

2.6.1. Auswertung der Atemwegscharakteristika................................................... 19

2.6.2. Endpunkte und Outcome-Variablen ............................................................... 19

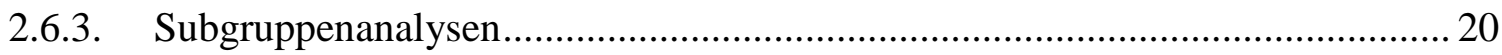

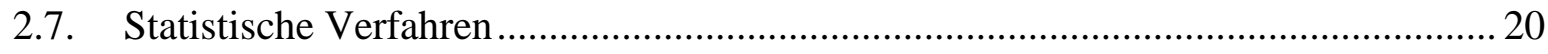

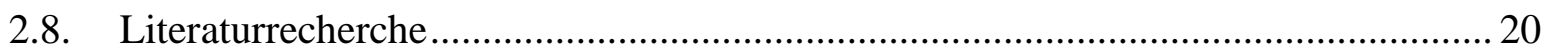




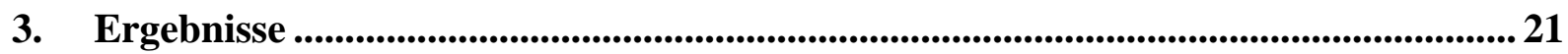

3.1. Demographische Daten und Einsatzcharakteristika ......................................... 22

3.1.1. Verteilung von Alter und Geschlecht.......................................................... 22

3.1.2. Anteil der Einsätze nach Rettungsmittel .................................................... 24

3.1.3. Eintreffzeiten und präklinische Versorgungsintervalle ................................... 25

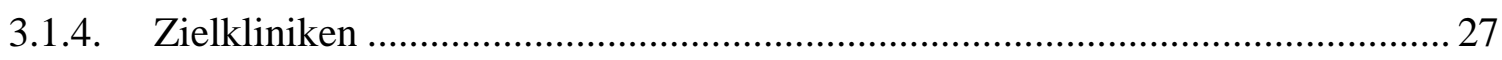

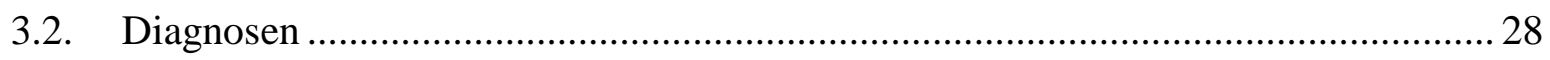

3.2.1. Schweregradeinschätzung anhand der NACA- und GCS-Scores ..................... 30

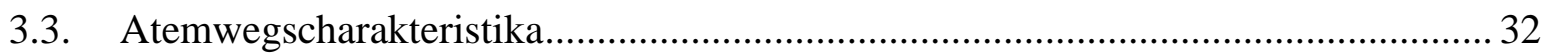

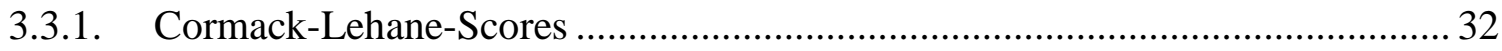

3.3.2. Inzidenz und Ursachen einer schwierigen Intubation ................................... 33

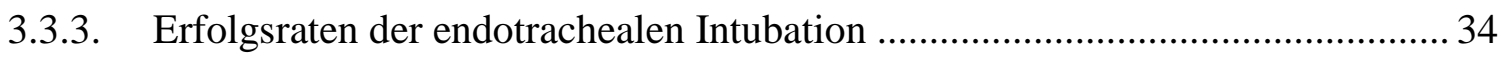

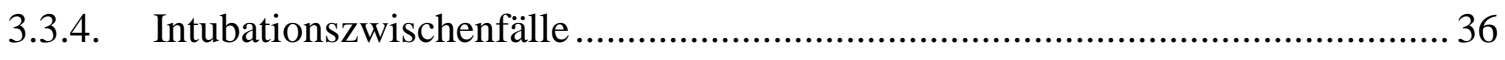

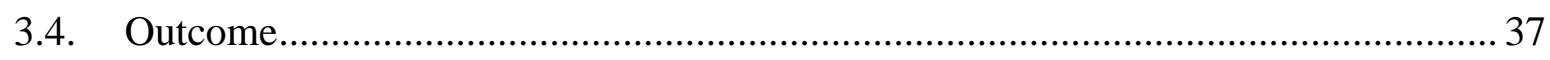

3.4.1. Dauer von Beatmung, Intensiv- und Krankenhausbehandlung ........................ 38

3.4.2. Neurologisches Outcome bei Krankenhausentlassung ................................. 40

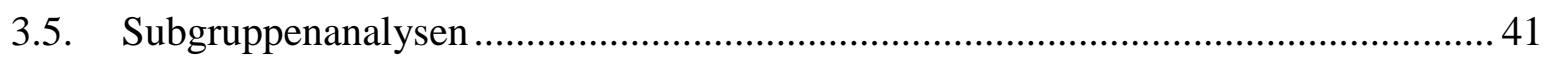

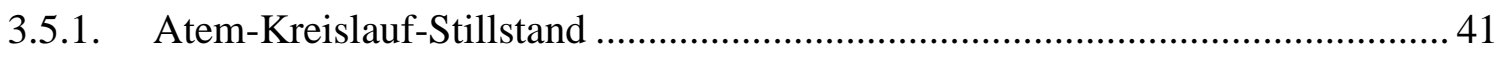

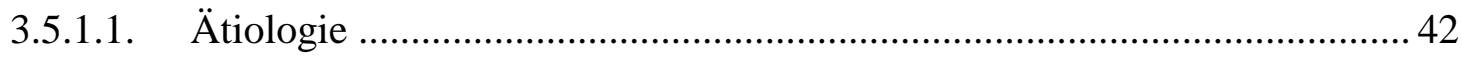

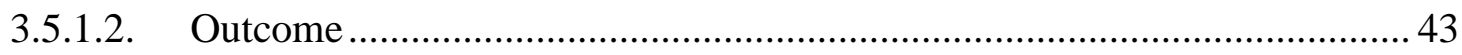

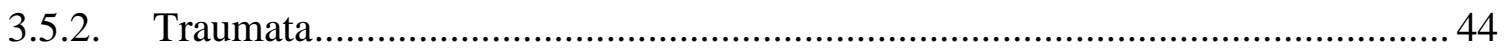

3.5.2.1. Ätiologie, Verletzungsmuster und -schwere ......................................... 44

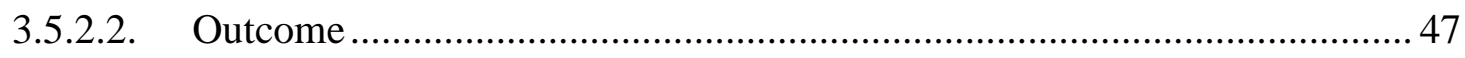

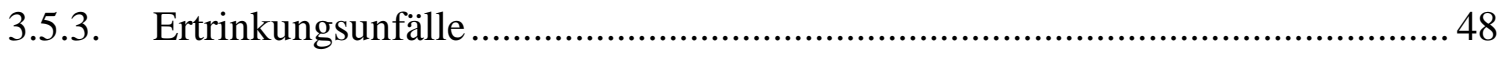

3.6. Wahrscheinlichkeit einer präklinischen Kinderintubation ................................... 49

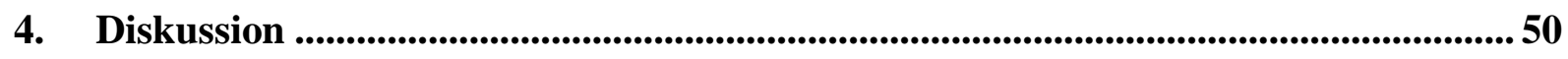

4.1. Charakteristika der präklinischen Intubation................................................. 50

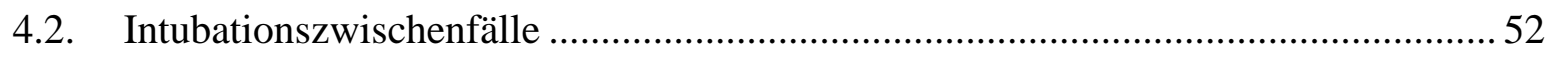


4.3. Einfluss der präklinischen Intubation auf das Outcome .......................................... 55

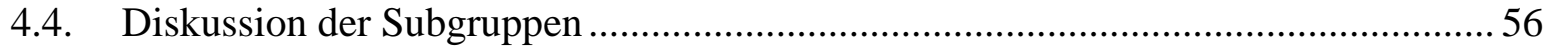

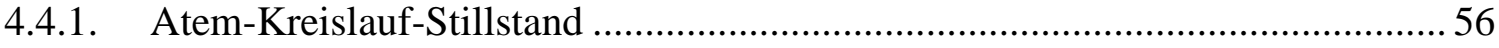

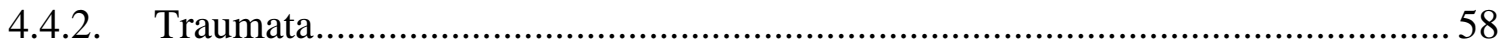

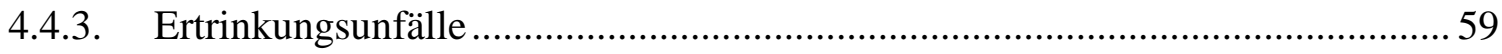

4.5. Die „Pros und Contras“ der präklinischen Intubation von Kindern ......................... 60

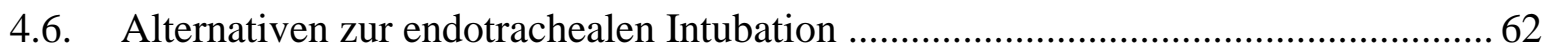

4.7. Strategien zur Aus-, Weiter- und Fortbildung im pädiatrischen

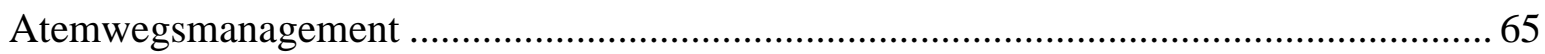

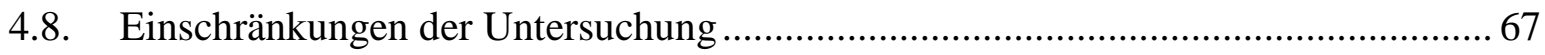

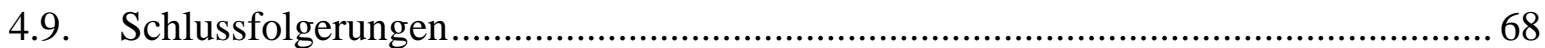

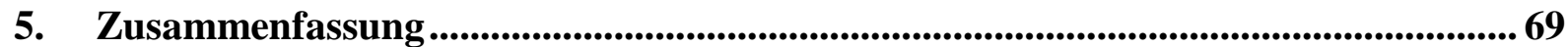

6. Anhang

6.1. Die RED ${ }^{\circledR}$-Eingabemaske zu den Charakteristika des Atemwegsmanagements ...... 70

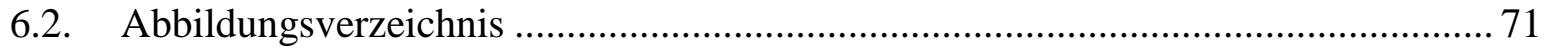

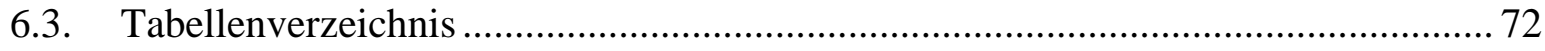

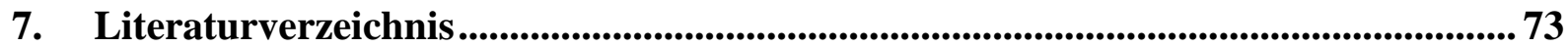




\section{Abkürzungsverzeichnis}

ALS

BLS

BMV

C2-C4

$\mathrm{CO}_{2}$

CPR

DIVI

EKG

engl.

EPLS

ERC

ETI

GCS

ILCOR

KI

NACA

NEF

OR

PCPC

PEA

$\mathrm{RED}^{\circledR}$

ROSC

RTH

SD

SGA

SHT

SIDS

$\mathrm{SpO}_{2}$

u.a.

vs.

ZARI
Advanced Life Support

Basic Life Support

Beutel-Masken-Ventilation

Zweiter bis vierter Halswirbelkörper

Kohlendioxid

Kardiopulmonale Reanimation

Deutsche Interdisziplinäre Vereinigung für Intensiv- und Notfallmedizin

Elektrokardiogramm

englisch

European Paediatric Life Support

European Resuscitation Council

Endotracheale Intubation

Glasgow-Coma-Scale

International Liaison Committee on Resuscitation

Konfidenzintervall

National Advisory Committee on Aeronautics

Notarzteinsatzfahrzeug

Odds Ratio

Paediatric Cerebral Performance Categories

Pulslose elektrische Aktivität

Rettungsdiensteinsatzdokumentations-Software des ZARI

Return Of Spontaneous Circulation

Rettungshubschrauber

Standardabweichung

Supraglottische Atemwegshilfen

Schädel-Hirn-Trauma

Sudden Infant Death Syndrome = plötzlicher Kindstod

Durch die Pulsoxymetrie ermittelte partielle Sauerstoffsättigung

unter anderem

versus

Zentrum Anaesthesiologie, Rettungs- und Intensivmedizin 


\section{Einleitung}

\subsection{Die Bedeutung der Atemwegssicherung bei Kindernotfällen}

Respiratorische Störungen bedingen einen großen Teil pädiatrischer Notfälle und stellen potenziell lebensbedrohliche Krankheitsbilder dar (HOFFMANN und NICOLAI (2009)). Die Sicherung der Atemwege gehört somit zu den wichtigsten Maßnahmen bei der präklinischen Versorgung von Kindern. In einer aktuellen Untersuchung von EICH et al. (2009b) aus dem Rettungsdienstbereich Göttingen liegt in über $20 \%$ der Notarzteinsätze bei Kindern eine primär respiratorisch bedingte Störung vor. Auch die Ätiologie des Herz-Kreislaufstillstandes im Kindesalter unterscheidet sich von der bei Erwachsenen, bei denen der Arrest in den meisten Fällen durch eine kardiale Arrhythmie ausgelöst wird. Bei Kindern bis zum Adoleszentenalter herrscht ein asphyktischer oder respiratorischer Herz-Kreislaufstillstand vor. Die Kausalkette, ausgehend von einer Hypoxie z.B. als Folge von Trauma, Ertrinken oder Vergiftung, führt über eine Bradykardie zur Asystolie (ENGDAHL et al. (2003)).

Die pädiatrische „Chain-of-Survival“ beinhaltet die vier ineinandergreifenden Glieder einer Kette, die bei der Versorgung kritisch kranker Kinder von essentieller Bedeutung sind, um die bestmöglichen Ergebnisse für Überlebensraten und Lebensqualität zu erreichen. Diese sind (1.) Prävention, (2.) frühestmöglicher Beginn von Basismaßnahmen, (3.) rasche Alarmierung und Verfügbarkeit des Rettungsdienstes und (4.) frühestmöglicher Beginn erweiterter lebensrettender Maßnahmen.

Abbildung 1: Chain-of-Survival aus ILCOR (2000), S. 345

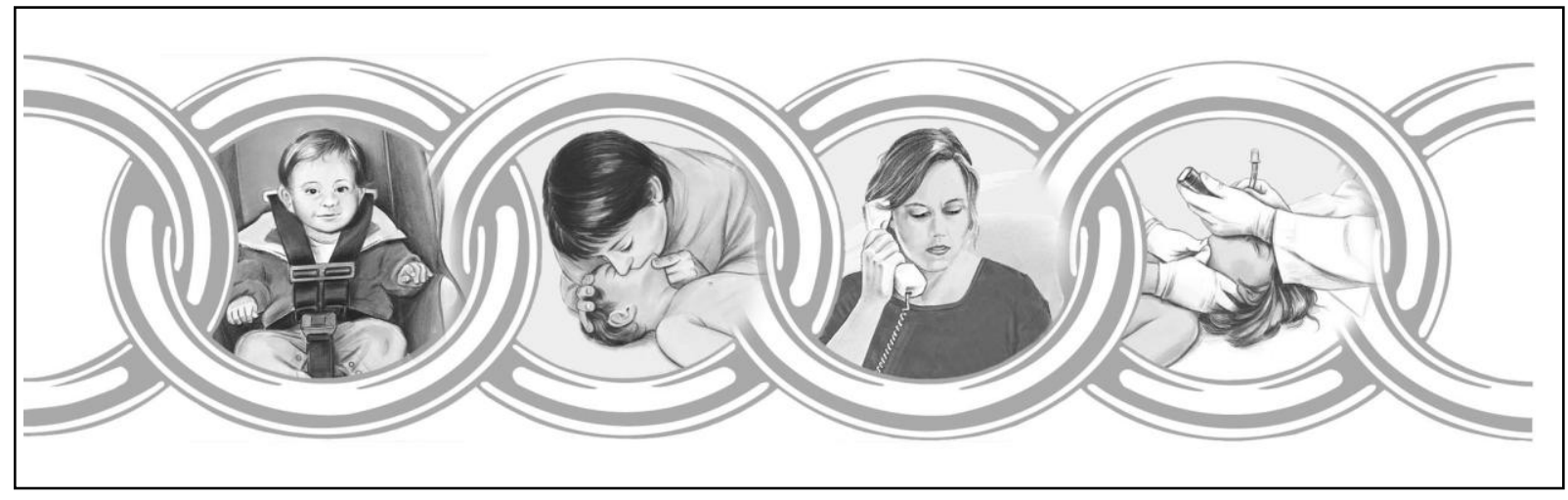

Das erste Glied der Rettungskette gilt der Prävention. Viele Ursachen pädiatrischer Notfälle sind durch präventive Maßnahmen vermeidbar. Lebensbedrohlichen Zuständen geht oftmals eine Phase physiologischer Instabilität voraus, welche - rechtzeitiges Erkennen vorausgesetztein Zeitfenster bietet zu intervenieren und das Outcome, d.h. das Behandlungsergebnis, zu verbessern (CHAPMAN et al. (2010)). Eine frühzeitige und adäquate Behandlung einer 
respiratorischen Insuffizienz ist eine der elementaren notfallmedizinischen (Präventiv-) Interventionen, um die Inzidenz des Atem-Kreislauf-Stillstandes im Kindesalter zu reduzieren (ZARITSKY et al. (1995)). In der Abbildung 1 symbolisiert die Durchführung einer Atemspende das zweite Glied der Rettungskette, das für den frühestmöglichen Beginn von Basismaßnahmen durch Ersthelfer steht (engl. Basic Life Support, BLS). Dies trägt der Tatsache Rechnung, dass Kreislaufstillstände bei Kindern primär meist respiratorisch bedingt sind und das Freimachen der Atemwege und die Behebung der Hypoxie die kausale Therapie darstellen. In den Leitlinien des European Resuscitation Council (ERC) für den pädiatrischen Atem-Kreislauf-Stillstand wird die Durchführung von BLS-Maßnahmen für einen Zeitraum von einer Minute empfohlen, ehe ein einzelner Helfer den Notruf absetzen sollte (BIARENT et al. (2005)). Diese Abfolge unterscheidet sich somit von der Chain-of-Survival für Erwachsene, bei denen frühzeitiger Notruf und die Defibrillation im Vordergrund stehen (HANDLEY et al. (2005)). Das letzte Glied der Kette steht für den frühestmöglichen Beginn erweiterter lebensrettender Maßnahmen (engl. Advanced Life Support, ALS). Auch hier wird die hohe Priorität der Sicherung der Atemwege und der Beatmung hervorgehoben und die endotracheale Intubation als Symbol für die ALS-Maßnahmen verwendet.

Eine zeitgerechte und adäquate Sicherung von Atemwegen und Oxygenierung ist insbesondere bei Kindern entscheidend für die weitere Prognose und beeinflusst maßgeblich das Outcome. Bei bedrohten oder verlegten Atemwegen können diese auf supraglottischer, glottischer oder infraglottischer Ebene durch den Einsatz verschiedener Hilfsmittel gesichert werden. Im präklinischen Alltag am häufigsten verbreitet ist die Anwendung der BeutelMasken-Ventilation (BMV), bestehend aus einem sich selbst-entfaltenden Beatmungsbeutel und einer Gesichtsmaske. Unterstützend können oro- oder nasopharyngeale Tuben (nach Guedel bzw. Wendl) eingeführt werden. Als supraglottische Atemwegshilfen (SGA) kommen im präklinischen Bereich beispielsweise Larynxmaske, Larynxtubus oder Combitubus zur Anwendung (YOUNGQUIST et al. (2007)). Eine definitive Atemwegssicherung mit bestmöglichem Schutz vor pulmonaler Aspiration von Mageninhalt bietet jedoch nur die Platzierung eines passenden Tubus in die Trachea im Rahmen der endotrachealen Intubation (ETI). Als Ultima Ratio kommt die Schaffung eines infraglottischen chirurgischen Atemweges in Form der Koniotomie oder Notfalltracheotomie in Betracht. 


\subsection{Besonderheiten des kindlichen Atemweges}

Viele Empfehlungen und Leitlinien für die präklinische Versorgung von Kindern werden aus

Daten von Erwachsenen extrapoliert. Jedoch ist die Übertragung von Erkenntnissen und Daten von Erwachsenen auf Kinder grundsätzlich limitiert durch grundlegende anatomische, physiologische und pathophysiologische Unterschiede.

Die kindlichen Atemwege weisen einige substantielle Besonderheiten auf, die am deutlichsten bei Neugeborenen und Säuglingen ausgeprägt sind (WALKER (2001), FREI et al. (2004)):

(1) Die Zunge ist relativ groß.

(2) Der Kopf ist relativ groß, der Hals kurz.

(3) Der Larynx ist höher positioniert.

(4) Die Epiglottis ist lang, weich und $\Omega$-förmig.

(5) Die Atemwege sind eng, weich und verletzlich.

(6) Die engste Stelle befindet sich subglottisch im Bereich des Ringknorpels.

(7) Die Trachea ist kurz.

(8) Rechter und linker Hauptbronchus entspringen beide im gleichen Winkel.

(9) Die funktionelle Residualkapazität ist klein, der Sauerstoffverbrauch hoch.

Diese Besonderheiten erfordern unterschiedliche Maßnahmen und Vorgehensweisen im Atemwegsmanagement von Kindern und können spezielle Probleme und Gefahren in sich bergen (HOLM-KNUDSEN und RASMUSSEN (2009)).

Die Gefahr der (oberen) Atemwegsobstruktion bei Kindern ist groß. Kleine Kinder haben in Relation zur Körpergröße einen großen Kopf und ein prominentes Okziput. Daher ist die Kopfposition für die Atemwege von enormer Bedeutung. In Rückenlage ist die optimale Lagerung des Kopfes zum Offenhalten der Atemwege bei Neugeborenen und Säuglingen die sogenannte Neutralposition, bei größeren Kindern die leichte Extension des Halses (PAAL et al. (2010b)). Hyperextension und -Flexion führen rasch zu einer Verlegung der Atemwege (JÖHR (2009)). Aufgrund der insgesamt engen und vulnerablen Atemwege kann es leicht zu bedrohlichen Komplikationen wie Laryngospasmus, Atemwegsödem oder -Trauma kommen.

Die Technik der endotrachealen Intubation bei Neugeborenen, Säuglingen und Kleinkindern unterscheidet sich in wichtigen Punkten von der bei größeren Kindern und Erwachsenen. Die Zunge ist relativ groß im Verhältnis zur Mandibula. Sie nimmt viel Raum in der Mundhöhle ein, was die Verdrängung durch den Laryngoskopspatel schwierig macht. 
Durch den bei Neugeborenen und Säuglingen hoch stehenden Larynx (C2-C4) und durch die Konfiguration der Epiglottis ist die Visualisierung der Stimmritze durch die konventionelle Laryngoskopie häufig schwierig. Es bietet sich eine modifizierte Laryngoskopie-Technik und gegebenenfalls die Verwendung gerader Laryngoskopspatel an (ADEWALE (2009)).

Abbildung 2: Laryngoskopie mit geradem Laryngoskopspatel aus ILCOR (2000), S. 354

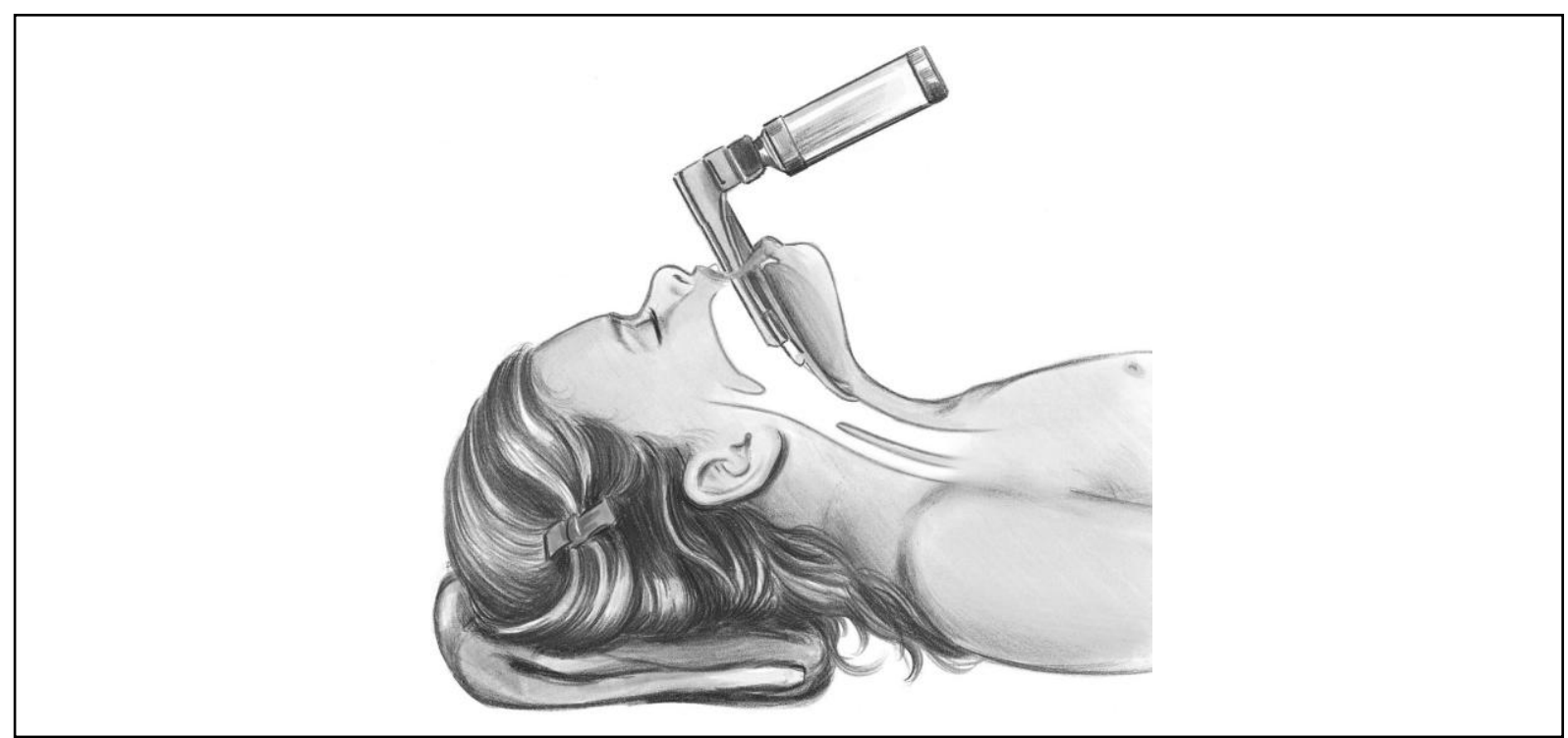

Bis zum Alter von zehn bis zwölf Jahren ist die engste Stelle der Luftwege im Bereich des Ringknorpels und nicht auf Stimmbandhöhe. Daher kann ein die Stimmlippen passierender Tubus zu groß für die subglottische Enge sein und ein zu forciertes Vorschieben zu einer Traumatisierung der Trachea führen. Die Trachea eines Neugeborenen ist mit etwa vier bis fünf Zentimeter kurz. Eine einseitige, also endobronchiale Intubation, aber auch eine versehentliche Tubusdislokation ist somit leicht möglich.

Kinder haben eine kleine funktionelle Residualkapazität und einen erhöhten Sauerstoffverbrauch $(5-8 \mathrm{ml} / \mathrm{kg} / \mathrm{min})$. Je kleiner die Kinder, desto geringer sind die Sauerstoffreserven. Eine lebensbedrohliche Hypoxämie tritt sehr schnell ein. In einer Simulationsstudie von HARDMAN und WILLS (2006), die die Apnoetoleranz bei gesunden, virtuellen Kindern (Alter: 1 Monat; 1, 8 und 18 Jahre) mittels eines validierten physiologischen Models untersuchte, konnte gezeigt werden, dass eine Hypoxämie bei kleineren Kindern dreimal schneller eintritt als bei älteren Kindern. Beim einmonatigen Kind trat die Sauerstoffentsättigung $\left(\mathrm{SpO}_{2}<90 \%\right)$ bereits nach 6,6 Sekunden auf, beim achtjährigen Kind nach 33,6 Sekunden.

Alle genannten Aspekte tragen dazu bei, dass das pädiatrische Atemwegsmanagement den Notarzt vor eine große Herausforderung stellen kann. 


\subsection{Die präklinische Intubation von Kindern}

Bei insuffizienter Atmung sowie verlegten oder bedrohten Atemwegen besteht die Notwendigkeit einer definitiven Kontrolle der Atemwege. Dazu stellt die endotracheale Intubation (ETI) nach weit verbreiteter Ansicht den Goldstandard der Versorgung dar (DOERGES et al. (2003b), BIARENT et al. (2005), HELM et al. (2006)). Ein wichtiges Ziel im Atemwegsmanagement von Kindern ist es, die endotracheale Intubation zeitgerecht, korrekt, sicher und zügig durchzuführen (WANG und YEALY (2006b)).

Die Intubation von Kindern erfordert schon unter Routinebedingungen ein großes Maß an Erfahrung und Expertise (STRAUSS und BECKE 2010)). Im Vergleich zum Innerklinischen ist das Atemwegsmanagement unter präklinischen Bedingungen weitaus schwieriger (BOSWELL et al. (1995), UMMENHOFER und SCHEIDEGGER (2002), TIMMERMANN et al. (2006)). Die präklinische endotracheale Intubation von Kindern stellt eine Herausforderung für das notfallmedizinische Personal dar und ist selbst für Anästhesisten mit fundierter Erfahrung im Atemwegsmanagement potenziell schwierig (DEAKIN (2000)). Sie ist eine relativ seltene und potenziell risikoreiche Maßnahme (HOLZKI et al. (2009)). Eine Vielzahl von Faktoren trägt dazu bei, dass die Intubation oftmals unter widrigen Bedingungen durchgeführt werden muss. Dazu zählen limitierte personelle und materielle Ressourcen, notwendige Immobilisation der Halswirbelsäule oder Behinderung der Sicht durch Blut und Erbrochenes in den oberen Atemwegen. Darüber hinaus sind nicht selten limitierende Umgebungsbedingungen wie schlechte Licht- und Platzverhältnisse oder Lärm anzutreffen (DORAN et al. (1995), DEAKIN (2000), MEYER et al. (2000), RICHARD et al. (2006)).

Verschiedene Untersuchungen zeigen auf, dass das klinische Outcome präklinisch intubierter Kinder vermeintlich nicht besser ist als das derjenigen Kinder, welche mittels Beutel-MaskenBeatmung ventiliert werden (GAUSCHE et al. (2000), COOPER A et al. (2001), EHRLICH et al. (2004), DIRUSSO et al. (2005), GERRITSE et al. (2008), LECKY et al. (2008)). Aufgrund des womöglich fehlenden Überlebensvorteils und einer möglicherweise hohen Rate an intubations-assoziierten Komplikationen werden Sinn und Nutzen der präklinischen Intubation in letzter Zeit kritisch hinterfragt. Manche Daten weisen sogar darauf hin, dass die präklinische Intubation mehr Schaden als Vorteile mit sich bringt (BURTON (2006)).

In Orange County im Ballungsraum Los Angeles, Californien/USA wurde die präklinische Intubation von Kindern nach einer großen randomisierten kontrollierten Studie von GAUSCHE et al. (2000) aus dem Kompetenzbereich der Paramedics entfernt. Auch in 
Großbritannien wurde die Empfehlung ausgesprochen, dass Paramedics sich auf die Anwendung von supraglottischen Atemwegshilfen beschränken sollten (DEAKIN et al. (2008)).

Die grundsätzliche Indikation zur endotrachealen Intubation kann in Einzelfällen in Frage gestellt werden, aber die eigentlichen Hauptdiskussionspunkte sind der richtige Zeitpunkt, Ort und Durchführende der Intubation (GAUSCHE et al. (2000), GERRITSE et al. (2008), EICH et al. (2010)). Die ETI kann eine lebensrettende Maßnahme bei kritisch kranken oder verletzten Kindern sein und einen positiven Einfluss auf das Outcome haben, vorausgesetzt sie wird zügig und sicher durchgeführt ohne sekundäre Schäden zu verursachen (ILCOR 2005)).

Die meisten bisher publizierten Studien untersuchen die ETI von Kindern in Paramedicbasierten Rettungssystemen ohne den präklinischen Einsatz von Notärzten (GAUSCHE et al. (2000), COOPER A et al. (2001), EHRLICH et al. (2004), DIRUSSO et al. (2005), GERRITSE et al. (2008)). Derzeit existieren nur wenige Publikationen über die präklinische Intubation von Kindern, wenn diese von anästhesiologisch weitergebildeten Notärzten durchgeführt werden (MEYER et al. (2000), SUOMINEN et al. (2000a), EHRLICH et al. (2004)).

\subsection{Fragestellungen}

Mit Hilfe der vorliegenden epidemiologischen Untersuchung sollen folgende Kernfragen beantwortet werden:

1. Welche Notfallsituationen führen zur Indikationsstellung präklinischer endotrachealer Intubationen von Kindern?

2. Welches sind die Charakteristika präklinischen Atemwegsmanagements bzw. von Intubationen bei Kindern, wenn sie von anästhesiologisch weitergebildeten Notärzten durchgeführt werden?

3. Wie ist das Outcome von Kindern nach präklinischer Intubation?

4. Wie häufig werden Notärzte mit einer präklinischen Intubation bei Kindern konfrontiert? 


\section{Material und Methoden}

\subsection{Studiendesign und Patientenkollektiv}

Wir führten eine prospektive Observationsstudie zur Erfassung und Analyse präklinischer endotrachealer Intubationen bei Kindern durch. Der Untersuchungszeitraum umfasste acht Jahre vom 01.10.2000 bis zum 30.09.2008. Da die Untersuchung nicht auf Versuchen basiert, die die Überprüfung durch das Ethik-Komitee der Medizinischen Fakultät erforderlich gemacht hätten und keine personenbezogenen Daten berichtet wurden, hat die EthikKommission keine formale Prüfung als erforderlich erachtet.

Eingeschlossen in die Studie wurden alle Kinder im Alter von 0 bis 14 Jahren, die im Beobachtungszeitraum präklinisch intubiert und von Notärzten des Zentrums für Anaesthesiologie, Rettungs- und Intensivmedizin (ZARI) der Universitätsmedizin Göttingen versorgt wurden. Kinder, bei denen die Intubation in einem primär versorgenden Krankenhaus durchgeführt wurde, wurden von der Studie ausgeschlossen.

Alle Kinder wurden entsprechend ihrem Alter einer der drei Altersgruppen zugeteilt:

(1) Säuglinge: 0 bis $1 \mathrm{Jahr}$

(2) Kleinkinder: 1 bis 5 Jahre

(3) Schulkinder: 6 bis 14 Jahre

Des Weiteren wurden alle erfassten Fälle einer der zwei Behandlungsgruppen zugeteilt:

(1) Gruppe 1: Intubation durch Notärzte des ZARI (Studienärzte)

(2) Gruppe 2: Intubation durch primärversorgende Notärzte und Weiterversorgung durch die Studienärzte 


\subsection{Struktur und Organisation des Notarztdienstes in Göttingen}

Das Göttinger Notarztsystem verfügt über zwei Notarzteinsatzfahrzeuge (NEF) und einen Rettungshubschrauber (RTH Christoph 44). Das jährliche Einsatzaufkommen dieser notarztbesetzten Rettungsmittel beträgt mehr als 4200 Einsätze. Die Bevölkerungszahl im Einsatzgebiet beziffert sich auf etwa 600.000 Einwohner auf einer Fläche von ca. 6000 Quadratkilometern (EICH et al. (2009b)). Der Anteil der unter 15-Jährigen an der Bevölkerung in der Stadt und im Landkreis Göttingen beträgt 13,2\% (GÖTTINGER STATISTISCHES INFORMATIONSSYSTEM (2008)).

Das Einsatzspektrum, demographische Kennzahlen und der geographische Versorgungsbereich des Göttinger Notarztsystems beinhalten sowohl Charakteristika des Rettungsdienstes einer mittelgroßen Stadt als auch eines ländlich geprägten Rettungsdienstes. Somit dürften Untersuchungen in diesem Rettungsdienstbereich den bundesdeutschen Durchschnitt in Bezug auf infrastrukturelle und geographische Aspekte reflektieren und in suffizienter Weise repräsentativ für Deutschland und andere zentraleuropäische Länder sein (EICH et al. (2009a)).

\subsubsection{Pädiatrische Notfallversorgung in der Stadt und Region Göttingen}

In den letzten Jahren wurden in Deutschland in einigen urbanen Ballungszentren spezielle Kindernotarztdienste etabliert (STENKE (2004), DIETZ und NICOLAI (2005)). Vielerorts musste dieser Service jedoch aus organisatorischen und finanziellen Gründen wieder eingestellt werden. In der Stadt und Region Göttingen wird kein spezialisiertes Kindernotarztsystem vorgehalten. Wie in den meisten Rettungsdienstbereichen in Deutschland sowie in vielen anderen Ländern Europas werden bei Kindernotfällen der Notarzt bzw. das Rettungsfachpersonal des regulären Rettungsdienstes alarmiert. In einer retrospektiven Analyse der Notarzteinsätze im Versorgungsbereich des Notarztdienstes Göttingen wurden von EICH et al. (2009b) im Neun-Jahres-Zeitraum von 1998 bis 2006 von insgesamt 36.240 Notarzteinsätzen 2271 Einsätze mit Kindern im Alter von 0 bis 14 Jahren identifiziert. Dies entspricht einem Jahresdurchschnitt von 252 Einsätzen und einem Anteil von 6,3\% pädiatrischer Notfälle per annum. Diese Fallzahlen stimmen mit den Angaben vergleichbarer Studien überein, die einen Bereich von 5,0 bis 12,9\% angeben, u.a. abhängig von der gewählten oberen Altersgrenze (SUOMINEN et al. (1996), ALBRECH et al. (2000), SCHLECHTRIEMEN et al. (2006)). 
In der Untersuchung von EICH et al. (2009b) zeigten sich u.a. zwei Charakteristika pädiatrischer Notfalleinsätze:

(1) Die Prävalenzen der Notfalldiagnosen zeigten, ähnlich wie in früheren Studien, eine klare altersabhängige Verteilung (ALBRECH et al. (2000), STENKE (2004), SCHLECHTRIEMEN et al. (2005)). In der Altersgruppe der Säuglinge waren respiratorische Störungen mit 34,5\% die führende Diagnose, bei den Kleinkindern Krampfanfälle mit 42,2\% und bei den Schulkindern Traumata mit 44,4\%. Diese drei führenden Diagnosegruppen machten zusammen mehr als $80 \%$ aller präklinischen Kindernotfälle aus.

(2) Die Schweregrade der kindlichen Notfälle gemessen an den NACA-Scores waren überwiegend relativ gering. So wurden $72,7 \%$ der Einsätze den NACA-Scores I-III zugewiesen.

\subsubsection{Notärzte}

Die Voraussetzung für die Tätigkeit als Notarzt ist die Erlangung der Zusatzbezeichnung Notfallmedizin, deren Inhalt in der Weiterbildungsordnung der jeweiligen Ärztekammern definiert wird (ROESSLER und ZUZAN (2006)).

Die ÄRZTEKAMMER NIEDERSACHSEN (2004) stellt folgende Voraussetzungen zum Erwerb der Zusatzbezeichnung Notfallmedizin:

- 24 Monate Weiterbildung in einem Gebiet der stationären Patientenversorgung bei einem Weiterbildungsermächtigten an einer Weiterbildungsstätte

- 6 Monate Weiterbildung in Intensivmedizin, Anästhesiologie oder in der Notfallaufnahme

- 80 Stunden Kurs-Weiterbildung in allgemeiner und spezieller Notfallbehandlung und anschließend

- 50 Einsätze im Notarztwagen oder Rettungshubschrauber unter Anleitung eines verantwortlichen Notarztes oder

- 20 Einsätze im Notarztwagen oder Rettungshubschrauber unter Anleitung eines Weiterbildungsermächtigten

Alle in der Studie eingeschlossenen Einsätze wurden von Notärzten, die dem Zentrum Anaesthesiologie, Rettungs- und Intensivmedizin (ZARI) der Universitätsmedizin Göttingen angehörten, geleitet (Studienärzte). Die eingesetzten Notärzte des ZARI sind entweder 
Fachärzte für Anästhesiologie oder befinden sich in fortgeschrittener Weiterbildung. Bevor diese am Notarztdienst der Abteilung teilnehmen, müssen sie mindestens zwei Jahre Tätigkeit in der klinischen Anästhesie, ein Jahr Intensivmedizin und die Zusatzbezeichnung Notfallmedizin nachweisen. Darüber hinaus müssen die Notärzte, um auf dem Rettungshubschrauber eingesetzt zu werden, wenigstens 300 Einsätze im bodengebundenen Rettungsdienst sowie eine 3 bis 4-monatige Rotation in der Kinderanästhesie absolviert haben.

In einigen Fällen waren Notärzte, welche nicht dem ZARI angehörten, als ersteintreffende Ärzte am Notfallort und führten die Primärbehandlung und ggf. die endotracheale Intubation durch, bis die Weiterversorgung und der Transport durch die Studienärzte übernommen wurde (Gruppe 2). Diese Notärzte - in allen Fällen von peripheren Notarztstützpunkten entstammten verschiedenen Disziplinen (v.a. Kollegen der Inneren Medizin und Chirurgie unterschiedlichen Ausbildungsstandes).

\subsection{Atemwegsinterventionen}

Die Indikation zur präklinischen Intubation lag in der Entscheidungsfreiheit der Notärzte, welche allgemein anerkannte Kriterien wie Glasgow-Coma-Scale $<$ 9, Polytrauma, Reanimation, etc. anwendeten. Die notfallmäßige Intubation wurde in der Regel als „Rapid Sequence Induction“ (RSI) nach Abteilungsstandard durchgeführt, mit Präoxygenierung gefolgt von der Gabe eines Opioids (Fentanyl) oder von S-Ketamin und eines Hypnotikums (Thiopental, Etomidate oder Midazolam), ggf. ergänzt durch ein Muskelrelaxans (Succinylcholin oder Rocuronium) (BECKE und SCHMIDT (2007)). Ein Studienprotokoll, welches den Einsatz oder die Dosierung von Narkosemedikamenten definitiv vorschreibt, existierte nicht. Kinder im Atem-Kreislauf-Stillstand und komatöse Patienten (GlasgowComa-Scale 3) wurden ohne Einsatz von Medikamenten intubiert. Die orotracheale Intubation war die präferierte Methode der Wahl. Die nasale Route war als alternative Technik gestattet, wenn diese vom Notarzt als geeignetes Verfahren betrachtet wurde, z.B. bei Neugeborenen und kleinen Säuglingen zur leichteren Tubusfixierung. Die korrekte Tubuslage und Beatmung wurde mittels Auskultation des Brustkorbes, Kapnographie und durch Kontrolllaryngoskopie mit Visualisierung des die Stimmlippen passierenden Tubus verifiziert.

Für den Fall einer schwierigen oder unmöglichen Intubation konnten alternative Atemwegshilfen eingesetzt werden. Zur Verfügung standen die supraglottischen Atemwegshilfen Larynxmaske (Classic ${ }^{\circledR}$ [alle Größen] und die Intubationslarynxmaske 
Fastrach $^{\circledR}$ [Größen 3-5]; LMA Deutschland GmbH, Bonn) sowie ein ösophagotrachealer Doppellumentubus (Combitube ${ }^{\circledR}$ [Größe 37 und 41 F]; Covidien Deutschland GmbH, Neustadt/Donau). Als Ultima-Ratio-Hilfsmittel zur Durchführung einer Koniotomie wurde ein Punktions-Cricothyrotomie-Set (Portex Minitrach Seldinger II ${ }^{\circledR}$; Smith Medical Deutschland GmbH, Grasbrunn) vorgehalten.

\subsection{Datenerfassung}

Patientenbezogene Daten wie Alter, Geschlecht, National Advisory Committee on Aeronautics (NACA) Score, Glasgow-Coma-Scale (GCS), Behandlungsdiagnose sowie einsatzbezogene Daten wurden unmittelbar nach jedem Einsatz von den Studienärzten dokumentiert und in ein elektronisches notfallmedizinisches Datenerfassungsprogramm eingegeben (Rettungsdiensteinsatzdokumentation, RED ${ }^{\circledR}$ ). Das RED $^{\circledR}$-Programm wurde eigens vom ZARI entwickelt und lizensiert. Weitere, ggf. im RED $^{\circledR}$-Datensatz nicht enthaltene Daten, wie z.B. detaillierte Informationen zum Notfallgeschehen, wurden den schriftlichen Notarzteinsatzprotokollen entnommen.

Retrospektiv wurden die medizinischen Krankenakten eingesehen, um die klinischen Verlaufsdaten zu erheben. Erfasst wurden die Überlebensdaten bis zur Krankenhausaufnahme und -entlassung, Diagnosen, Todesursachen, Intensivbehandlungs- und Beatmungsdauer, Entlassungsziel und neurologischer Status kurz vor der Klinikentlassung.

Die Inhalte und Definitionen der gesammelten Daten sind konsistent mit den internationalen Konsensusempfehlungen zur Erfassung erweiterter pädiatrischer lebensrettender Maßnahmen (ZARITSKY et al. (1995)): „Recommended Guidelines for Uniform Reporting of Pediatric Advanced Life Support: the Pediatric Utstein Style."

Sämtliche Daten wurden in einer elektronischen Datenbank (Excel 2007 ${ }^{\circledR}$, Microsoft Corporation, Redmond, USA) zusammengefasst. 


\subsubsection{Erfassung der Atemwegscharakteristika}

Die in der Folge genannten Atemwegscharakteristika waren nach Beendigung des Rettungseinsatzes von den Notärzten selbst in einer speziell für diese Studie erstellten Eingabemaske des RED $^{\circledR}$-Programms zu dokumentieren, bevor die Falldokumentation abgeschlossen werden konnte (siehe Anhang):

(1) Bei Durchführung der Laryngoskopie war die Sicht auf die Glottis entsprechend dem Cormack-Lehane Scoring-System zu ermitteln.

(2) Darüber hinaus mussten die Notärzte eine subjektive Einschätzung vornehmen, ob eine schwierige Intubation vorlag oder nicht.

(3) Es wurden die Intubationsroute,

(4) die Anzahl der Intubationsversuche,

(5) bestehende und/oder erschwerende klinische Umstände und

(6) der eventuelle Einsatz alternativer Atemwegshilfen abgefragt. 


\subsubsection{Schweregrad- und Outcome-Erfassung mittels Scoring-Systemen}

\subsubsection{Cormack-Lehane-Score}

Der Cormack-Lehane (CL) -Score wurde als Prädiktor für die schwierige Intubation unter Krankenhaus-Bedingungen validiert (CORMACK und LEHANE (1984)). Er beschreibt die Sichtverhältnisse des Intubateurs auf die anatomischen Strukturen bei der direkten Laryngoskopie und unterteilt diese entsprechend in vier Grade.

Über die präklinische Anwendung des CL-Score gibt es nur wenige Veröffentlichungen (ELGANZOURI et al. (1996), TIMMERMANN et al. (2006)). Für den pädiatrischen Bereich sind nach Kenntnis des Verfassers bisher keine präklinischen Untersuchungen beschrieben worden, jedoch hat sich der CL-Score im klinischen Bereich in der Kinderanästhesie fest etabliert (MACNAIR et al. (2009), VLATTEN et al. (2009)). Eine schwierige Intubation in der konventionellen Laryngoskopie liegt bei den Cormack-Lehane-Graden III und IV vor.

Tabelle 1: Definition der laryngoskopischen Sicht nach CORMACK und LEHANE (1984)

\begin{tabular}{|l|l|}
\hline Grad I & Larynxeingang vollständig sichtbar \\
\hline Grad II & Nur hinterer Teil des Larynxeinganges sichtbar \\
\hline Grad III & Nur Epiglottis sichtbar \\
\hline Grad IV & Nur weicher Gaumen sichtbar \\
\hline
\end{tabular}

Abbildung 3: Cormack-Lehane-Skala aus SAMSOON und YOUNG (1987), S. 488

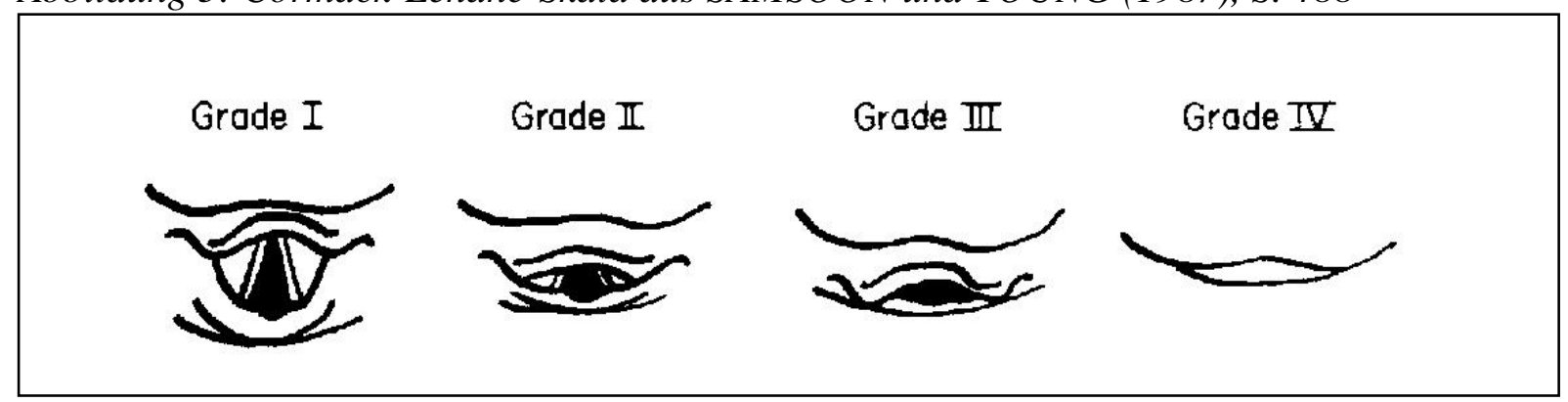




\subsubsection{National Advisory Committee on Aeronautics-Index (NACA)}

Der modifizierte National Advisory Committee on Aeronautics-Index (NACA) nach TRYBA et al. (1980) ist ein einfaches und adäquates Instrument zur Beschreibung der Vitalgefährdung von Notfallpatienten. Die Erfassung des NACA-Index gehört bei der Dokumentation von Notfalleinsätzen auf den bundesweit verwendeten DIVI-Notarzteinsatzprotokollen zum Kerndatensatz.

Der NACA-Index ist gekennzeichnet durch eine gute Übereinstimmung mit der zu erwartenden Morbidität und Mortalität (WEISS et al. (2001a), SCHLECHTRIEMEN et al. (2005)). Anhand des NACA-Indexes kann man die Notfälle im Rahmen der Ersteinschätzung in weniger schwerwiegend (NACA I-III) und Notfälle mit akuter Vitalgefährdung (NACA IV-VI) bzw. bereits eingetretenem Tod (NACA VII) unterteilen (EICH et al. (2009b)).

Tabelle 2: Modifizierter NACA-Index nach Tryba

aus SCHLECHTRIEMEN et al. (2005), S. 99

\begin{tabular}{|l|l|}
\hline NACA I & Geringfügige Störung \\
\hline NACA II & Ambulante Abklärung \\
\hline NACA III & Stationäre Behandlung \\
\hline NACA IV & Akute Lebensgefahr nicht auszuschließen \\
\hline NACA V & Akute Lebensgefahr \\
\hline NACA VI & Reanimation \\
\hline NACA VII & Tod \\
\hline
\end{tabular}




\subsubsection{Glasgow-Coma-Scale (GCS)}

Die Glasgow-Coma-Scale (GCS) nach TEASDALE und JENNETT (1974) ist ein international angewandtes Scoring-System zur Einschätzung des Schweregrades bei SchädelHirn-Trauma (SHT). Die GCS basiert auf der standardisierten Beurteilung der drei Qualitäten (1) Öffnen der Augen, (2) verbale Antwort und (3) motorische Antwort (BRAIN TRAUMA FOUNDATION (2007)).

Ursprünglich als Scoring-System für das Schädel-Hirn-Trauma entwickelt und validiert, hat sich die GCS in der präklinischen Notfallmedizin als universelles Punktesystem zur Quantifizierung der Bewusstseinslage und zur Einschätzung einer zerebral bedingten vitalen Gefährdung etabliert (MERKENSCHLAGER (2009)). Die GCS hat sich dabei als physiologisches Messinstrument mit hohem prädiktiven Wert zur Identifizierung vital bedrohter Kinder erwiesen (NEWGARD et al. (2007)). Auf dem DIVI-Notarztprotokoll gehört die Dokumentation der GCS zum Kerndatensatz (MOECKE et al. (2000)).

Der GCS stellt einen Summenwert der jeweiligen Punkte der drei o.g. Qualitäten dar mit einem Maximum von 15 und einer minimal möglichen Punktzahl von drei (entsprechend komatös). Anhand der GCS-Punkte wird das Schädel-Hirn-Trauma in drei Schweregrade eingeteilt (FIRSCHING et al. (1996)):

(1) GCS 13-15 Punkte: Leichtes SHT

(2) GCS 9-12 Punkte: Mittelschweres SHT

(3) GCS 3-8 Punkte: Schweres SHT

Die GCS hat sich als valider Marker des cerebralen Hypoxierisikos bei Schädel-Hirn-Trauma erwiesen (STEIN und SPETTELL (1995)). Die repetitiv durchgeführte GCS erlaubt die Erkennung und Beschreibung einer enzephalopathischen Progredienz und der damit verbundenen vitalen Gefährdung.

Der empfohlene Schwellenwert für eine endotracheale Intubation beim SHT beträgt unabhängig vom Alter < 9 Punkte (WAGNER (2009)).

Bei Kleinkindern und Säuglingen lassen sich die verbalen und motorischen Antworten des originären GCS nicht ohne Weiteres anwenden. Für diese Altersklassen existieren adaptierte pädiatrische GCS-Tabellen (SIMPSON et al. (1991)). 
Tabelle 3: Glasgow-Coma-Scale für Erwachsene und Kinder > 5 Jahre aus WAGNER (2009), S. 134

\begin{tabular}{|l|l|c|}
\hline \multirow{5}{*}{ Augenöffnen } & Kein & 1 \\
\cline { 2 - 3 } & Auf Schmerz & 2 \\
\cline { 2 - 3 } & Auf Ansprache & 3 \\
\cline { 2 - 3 } & Spontan & 4 \\
\hline \multirow{5}{*}{ Beste verbale Antwort } & Keine & 1 \\
\cline { 2 - 3 } & Unverständliche Laute & 2 \\
\cline { 2 - 3 } & Unzusammenhängende Laute & 3 \\
\cline { 2 - 3 } & Verwirrt & 4 \\
\cline { 2 - 3 } & Orientiert & 5 \\
\hline & Keine & 2 \\
\cline { 2 - 3 } & Extension aller Extremitäten & 3 \\
\cline { 2 - 3 } & Abnorme Flexion mit Pronation der oberen Extremität & 5 \\
\cline { 2 - 3 } & Ungezielte Abwehr/normale Flexion der oberen Extremität & 4 \\
\cline { 2 - 3 } & Gezielte Schmerzabwehr & 6 \\
\cline { 2 - 3 } & Auf Aufforderung & 5 \\
\hline
\end{tabular}

Tabelle 4: Glasgow-Coma-Scale für Kinder < 5 Jahre

aus WAGNER (2009), S. 134

\begin{tabular}{|l|l|c|}
\hline \multirow{5}{*}{ Augenöffnen } & Kein & 1 \\
\cline { 2 - 3 } & Auf Schmerz & 2 \\
\cline { 2 - 3 } & Auf Ansprache & 3 \\
\cline { 2 - 3 } & Spontan & 4 \\
\hline \multirow{5}{*}{ Beste verbale Antwort } & Keine & 1 \\
\cline { 2 - 3 } & Stöhnen auf Schmerz & 2 \\
\cline { 2 - 3 } & Schreien auf Schmerz & 3 \\
\cline { 2 - 3 } & Spontanes Schreien & 4 \\
\cline { 2 - 3 } & Spontanes Lautgeben, Plappern & 5 \\
\hline & Keine & 2 \\
\cline { 2 - 3 } & Extension auf Schmerzreiz & 3 \\
\cline { 2 - 3 } & Abnorme Flexion mit Pronation der oberen Extremität & 5 \\
\cline { 2 - 3 } & Ungezielte Abwehr/normale Flexion der oberen Extremität & 4 \\
\cline { 2 - 3 } & Gezielte Schmerzabwehr & 6 \\
\cline { 2 - 3 } & Normales spontanes Bewegungsmuster & 5 \\
\hline
\end{tabular}




\subsubsection{Paediatric Cerebral Performance Category (PCPC)}

Der neurologische Status bei Krankenhausentlassung wurde mittels Erhebung der Paediatric Cerebral Performance Category (PCPC) (Kategorie 1-5) graduiert. Diese stellt eine pädiatrische Modifikation der Glasgow-Pittsburgh Outcome Categories nach JENNETT und BOND (1975) dar, welche bei Erwachsenen die am meisten verbreitete Klassifikation zur Evaluierung der Lebensqualität nach erfolgreicher Reanimation ist.

Die PCPC-Skalen wurden zur Erfassung kognitiver Beeinträchtigungen und funktionaler Morbidität kritisch erkrankter oder verletzter Kinder entwickelt. Sie stellen reliable und valide Methoden zur Quantifizierung des funktionellen Outcomes in der pädiatrischen Intensivmedizin dar (FISER (1992)). Die PCPC-Punktewerte ergeben wertvolle Informationen über das zu erwartende Outcome pädiatrischer Intensivpatienten, wenn komplexe psychometrische Tests nicht praktikabel oder sinnvoll durchführbar sind (FISER et al. (2000)). Die zu erhebenden Befunde sind einfach zu ermitteln und können beispielsweise durch ein Telefongespräch mit den Eltern erfragt werden (ZARITSKY et al. (1995)). Die PCPC-Werte können zu den Zeitpunkten prä-Ereignis und nach Krankenhausentlassung erfasst werden. Die Differenz zwischen prä-Ereignis-Status und bei Krankenhausentlassung wird als Delta-Score definiert.

In der vorliegenden Untersuchung wurde das neurologische Outcome in eine dichotome Variable umgewandelt. PCPC 1-3 oder keine Änderung zum vorbestehenden neurologischen Status (Delta-Score $=0$ ) wurden als gutes neurologisches Outcome, wohingegen die PCPC Grade 4-6 als schlechtes neurologisches Outcome definiert wurden (GAUSCHE et al. (2000), WU et al. (2009)). 
Tabelle 5: Paediatric Cerebral Performance Category Scale (PCPC)

modifiziert aus FISER (1992), S. 69

\begin{tabular}{|l|l|l|}
\hline Score & Kategorie & Beschreibung \\
\hline 1 & Normal & Altersentsprechend \\
\hline 2 & Leichte Behinderung & $\begin{array}{l}\text { Bei Bewusstsein; in der Lage altersentsprechend zu } \\
\text { interagieren. Schulkinder, die eine reguläre Schulklasse } \\
\text { besuchen, aber unterdurchschnittliche kognitive } \\
\text { Fähigkeiten aufweisen (mildes neurologisches Defizit) }\end{array}$ \\
\hline 3 & Moderate Behinderung & $\begin{array}{l}\text { Bei Bewusstsein; suffiziente cerebrale Funktionen für } \\
\text { altersgemäße Aktivitäten des täglichen Lebens. } \\
\text { Schulkinder, die spezielle Förderklassen besuchen } \\
\text { und/oder Lerndefizite vorweisen }\end{array}$ \\
\hline 4 & Schwere Behinderung & $\begin{array}{l}\text { Bei Bewusstsein; abhängig von Anderen in alltäglichen } \\
\text { Lebenssituationen aufgrund einer beeinträchtigten } \\
\text { Hirnfunktion }\end{array}$ \\
\hline 5 & $\begin{array}{l}\text { Koma / } \\
\text { Vegetativer Status }\end{array}$ & $\begin{array}{l}\text { Jeder Grad von Koma ohne die Kriterien des Hirntodes } \\
\text { zu erfüllen; kein Bewusstsein, keine Interaktion mit der } \\
\text { Umgebung; erloschene kortikale Funktionen; } \\
\text { reflektorische Antworten möglich, z.B. Augenöffnen, } \\
\text { Schlaf-Wach-Zyklen } \\
\text { Apnoe, Areflexie und/oder Nulllinien-EEG }\end{array}$ \\
\hline
\end{tabular}




\subsection{Datenschutz}

Es wurden keine personenbezogenen Daten gespeichert. Die Daten wurden erst verwendet, nachdem sie anonymisiert worden waren.

\subsection{Datenauswertung}

Alle gesammelten Daten wurden aus der $\mathrm{RED}^{\circledR}$-Datenbank extrahiert, aufbereitet und mit Hilfe eines Tabellenkalkulationsprogramms (Excel $2007^{\circledR}$, Microsoft Corporation, Redmond, USA) archiviert und für die weitere Analyse vorbereitet.

\subsubsection{Auswertung der Atemwegscharakteristika}

Die Auswertung der atemwegs-bezogenen Daten umfasste den Schwierigkeitsgrad der Intubation (Cormack-Lehane-Score und subjektive Einschätzung), die Erfolgsraten und die Anzahl der benötigten Intubationsversuche. Valide Dokumentationen dieser Kriterien lagen nur in den Fällen der Gruppe 1 (Intubation durch Studienärzte) vor.

Des Weiteren wurden die Daten hinsichtlich intubations-assoziierter Komplikationen für beide Gruppen überprüft. Diese im Folgenden als Intubationszwischenfälle bezeichneten Komplikationen lagen vor, wenn einer oder mehrere von drei Schlüsselfehlern dokumentiert wurden (WANG et al. (2009a)):

1. Tubusfehllage (ösophageal, unerkannt endobronchial) oder Tubusdislokation

2. Multiple Intubationsversuche ( $\geq 3$ Laryngoskopien)*

3. Misslungene Intubation

Die Häufigkeiten der Intubationszwischenfälle in beiden Gruppen wurden miteinander verglichen.

Als Intubationsversuch wurde die Insertion des Laryngoskopspatels gewertet (*).

\subsubsection{Endpunkte und Outcome-Variablen}

Die Überlebensrate bei Krankenhausentlassung war der primäre, der neurologische Status bei Krankenhausentlassung der sekundäre Endpunkt. Beide Parameter sind bedeutend für die Erfassung des Langzeitüberlebens und der Lebensqualität (GAUSCHE-HILL et al. (2000)). 
Zusätzliche Outcome-Variablen wie Überlebensrate bis zur Krankenhauseinweisung, Behandlungsdauer auf der Intensivstation/im Krankenhaus oder Beatmungsdauer ermöglichen einen weiteren Vergleich mit anderen Studien.

\subsubsection{Subgruppenanalysen}

Da es in der vorhandenen Literatur Hinweise dafür gibt, dass die präklinische ETI das Outcome spezieller Populationen von pädiatrischen Notfallpatienten beeinflussen könnte, wurde das Studienkollektiv gesondert für drei Subgruppen analysiert. Im Diskussionsteil wird auf einen Vergleich mit entsprechenden Publikationen eingegangen:

(1) Außerklinischer Atem-Kreislauf-Stillstand

(2) Traumata

(3) Ertrinkungsunfälle.

\subsection{Statistische Verfahren}

Die Auswertung der gewonnen Daten erfolgte überwiegend deskriptiv. Statistische Analysen wurden mit Hilfe von SPSS Statistics ${ }^{\circledR}$ (SPSS Inc., Chicago, IL, USA) und Excel $2007^{\circledR}$ (Microsoft Corporation, Redmond, USA) durchgeführt. Bei der Analyse metrischer Daten wurden sowohl arithmetischer Mittelwert \pm Standardabweichung als auch Median und Bereich berechnet. Bei der Deskription nicht-normalverteilter Daten wurden Median und Bereich angegeben. Zur Untersuchung von Unterschieden zwischen kategorialen Variablen (Korrelation der Behandlungsgruppen) wurde der Exakte Test nach Fisher angewendet. Zur Signifikanztestung der Alterseffekte setzten wir den Spearman's-Rho-Test ein. Ein zweiseitiger Wahrscheinlichkeitswert (p) von $\leq 0,05$ wurde als statistisch signifikant gewertet.

\subsection{Literaturrecherche}

Es erfolgte eine selektive Literaturrecherche mittels der medizinischen Datenbanken PubMed (U.S. National Library of Medicine), Cochrane und Ovid mit Hilfe von Referenzen aus relevanten Publikationen sowie unter Einbeziehung aktueller themenrelevanter Richtlinien, Leitlinien und Empfehlungen. 


\section{Ergebnisse}

Während des achtjährigen Studienzeitraumes wurden insgesamt 2040 präklinische Kindernotfälle durch die Göttinger Notärzte versorgt. 82 Kinder (4,0\%) wurden präklinisch endotracheal intubiert. 58 Kinder wurden durch die Studiennotärzte intubiert (Gruppe 1), 24 Kinder durch Nicht-Studiennotärzte, die primär am Notfallort die Behandlung begannen (Gruppe 2).

Abbildung 4: Flussdiagramm zum Studienkollektiv

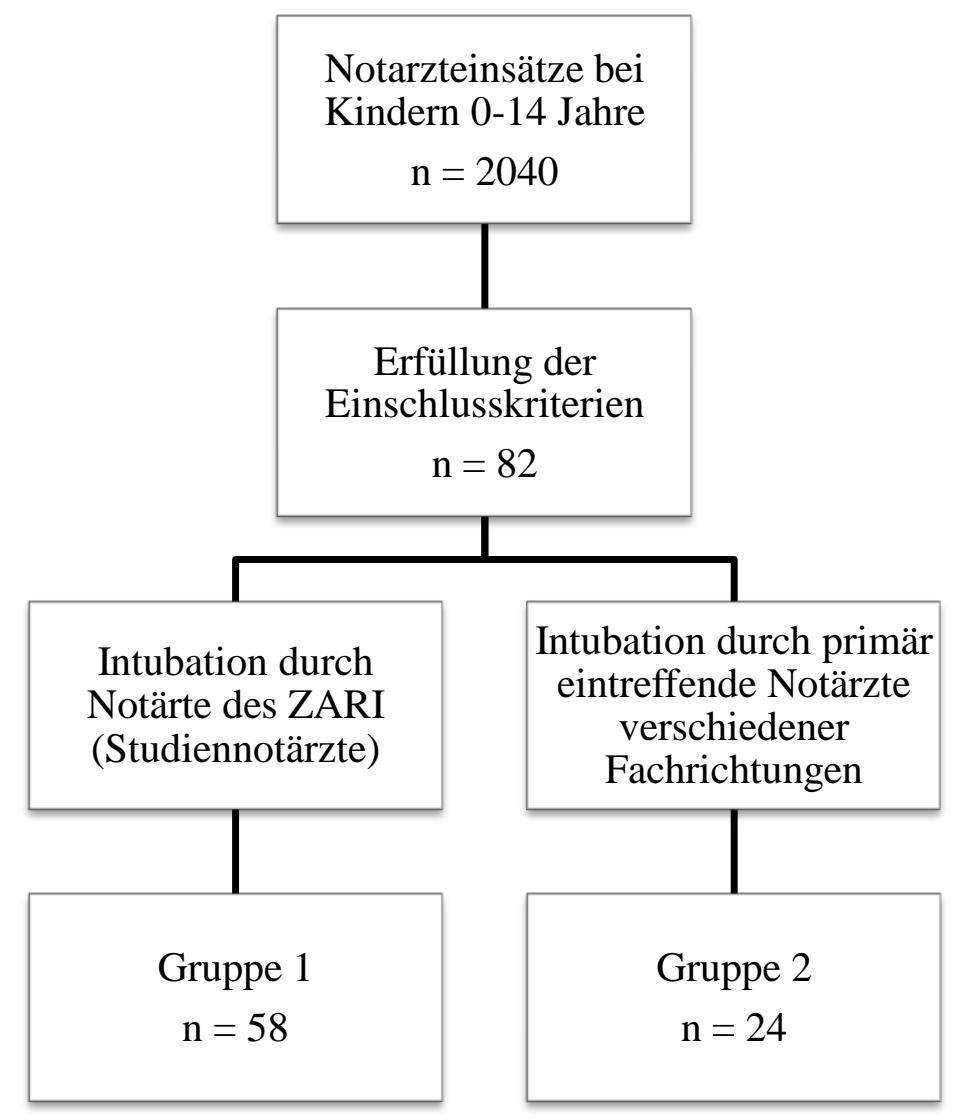




\subsection{Demographische Daten und Einsatzcharakteristika}

\subsubsection{Verteilung von Alter und Geschlecht}

Abbildung 5 gibt einen Überblick über die Altersverteilung der im Studienkollektiv eingeschlossenen intubierten Kinder $(n=82)$. Im Median waren die Kinder 4 Jahre alt (Mittelwert 5,5 \pm Standardabweichung 4,7).

\section{Abbildung 5: Balkendiagramm der Altersverteilung}

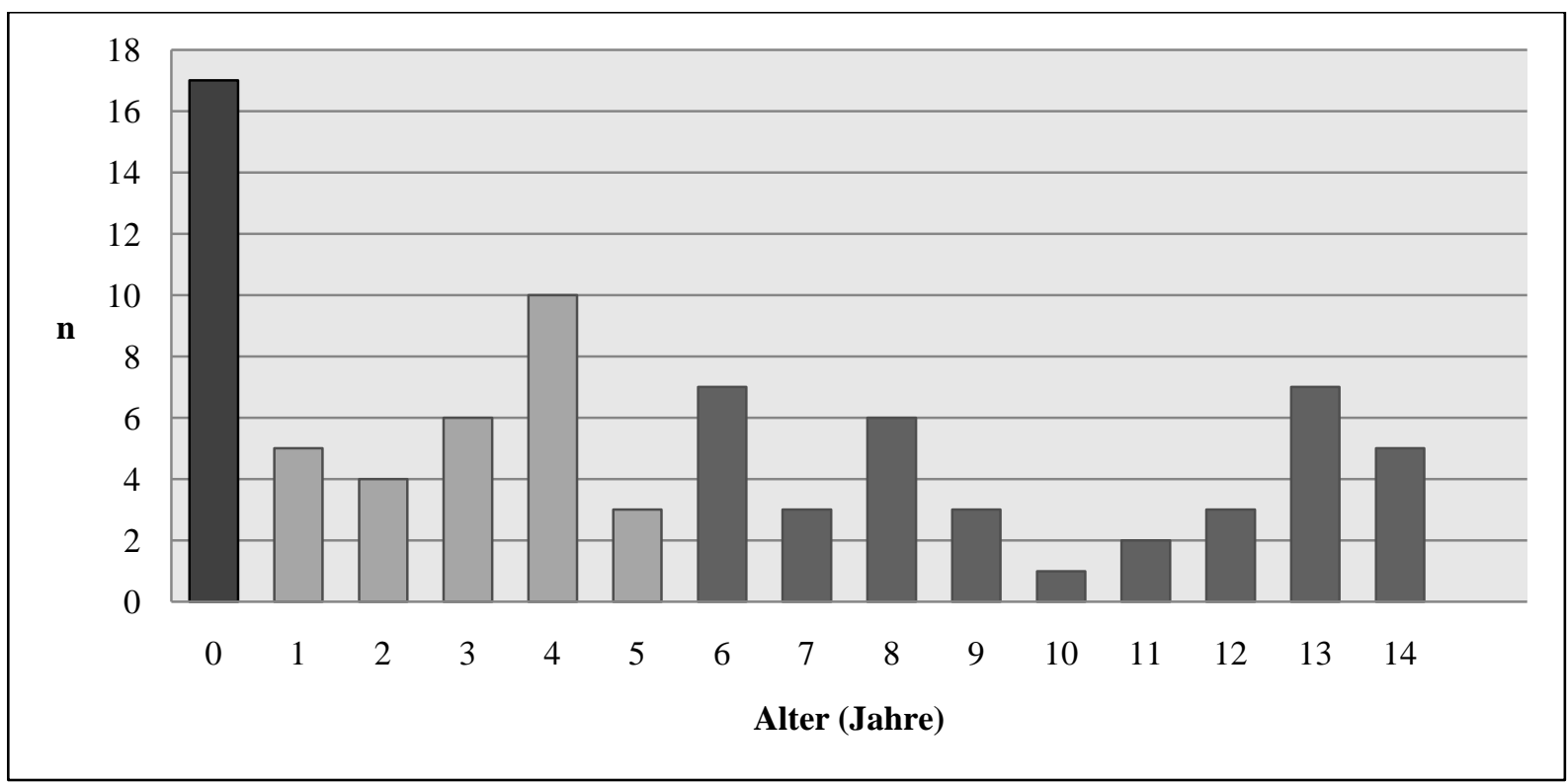

In der Verteilung nach den drei Altersgruppen erfüllten 17 Säuglinge (20,7\%), 28 Kleinkinder $(34,1 \%)$ und 37 Schulkinder $(45,1 \%)$ die Einschlusskriterien.

Abbildung 6: Balkendiagramm der Altersgruppen

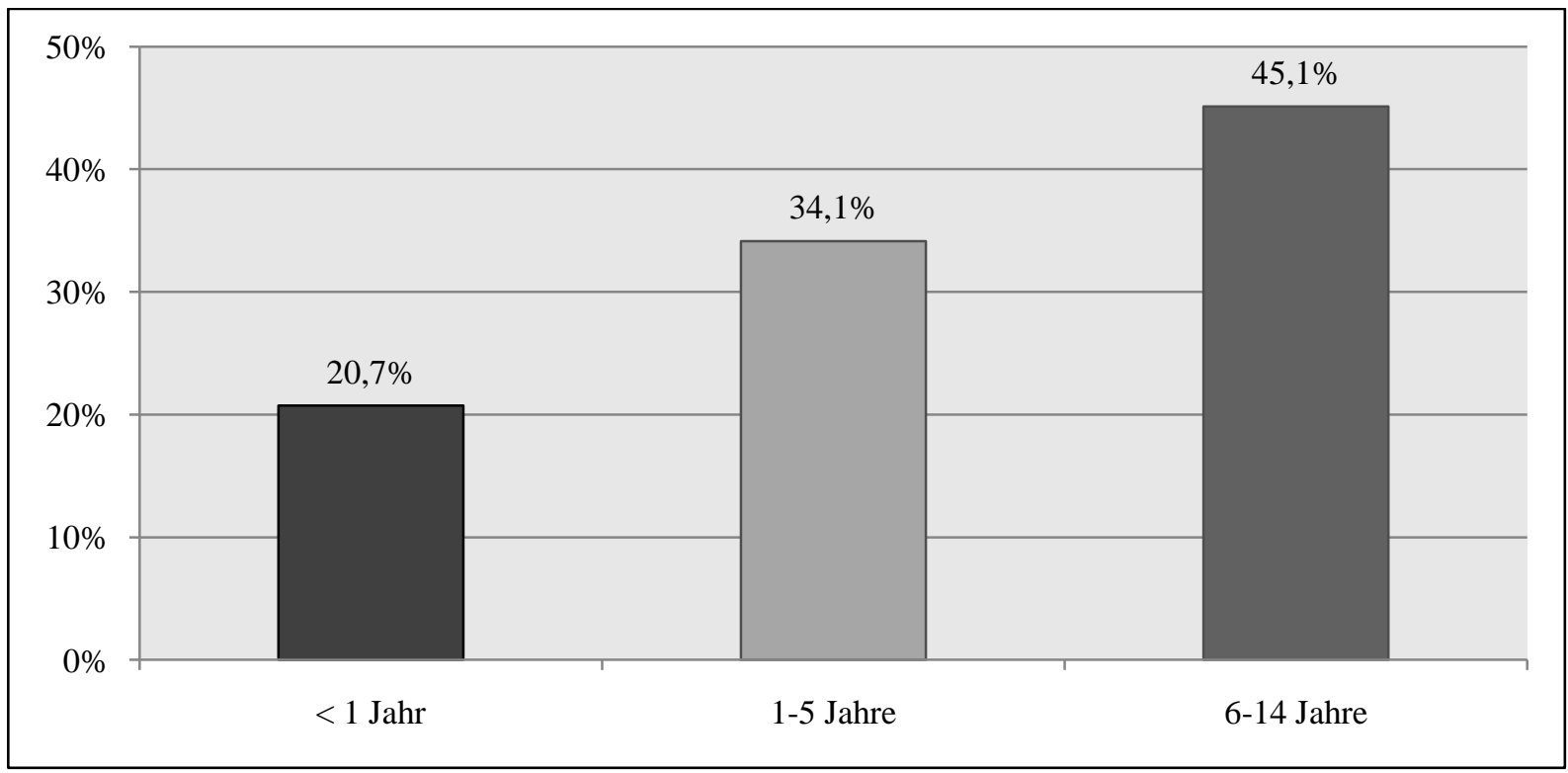


Die Mehrheit der präklinisch intubierten Kinder waren mit 56 Fällen Jungen ( $₫$ 68,3\%). Der Anteil der Mädchen betrug 26 ( $31,7 \%$ ). Auch auf die drei Altersgruppen betrachtet, zeigt das männliche Geschlecht den dominierenden Anteil (Tabelle 6).

Abbildung 7: Geschlechterverteilung

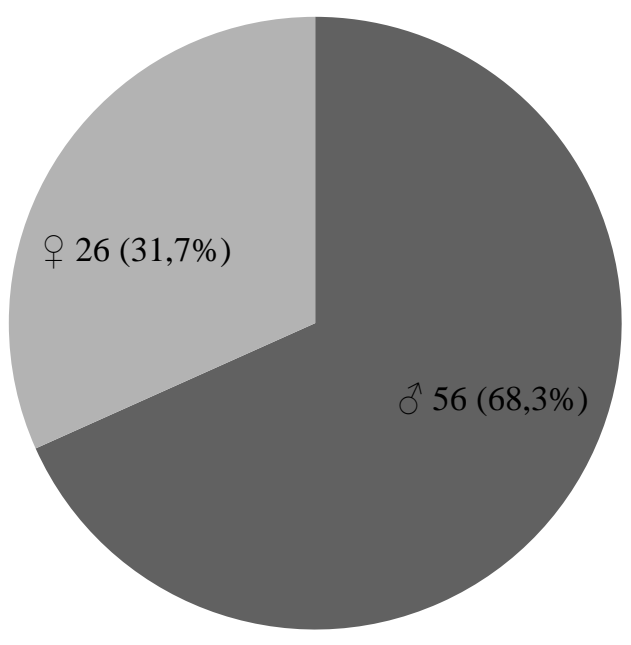

Tabelle 6: Geschlechterverteilung in Abhängigkeit der Altersgruppen

\begin{tabular}{lllll}
\hline & $<1 \mathrm{Jahr}$ & 1-5 Jahre & 6-14 Jahre & Alle $(\%)$ \\
Geschlecht & $\mathrm{n}=17(\%)$ & $\mathrm{n}=28(\%)$ & $\mathrm{n}=37(\%)$ & $\mathrm{n}=82(\%)$ \\
\hline Männlich & $11(64,7)$ & $20(71,4)$ & $25(67,6)$ & $56(68,7)$ \\
Weiblich & $6(35,3)$ & $8(28,6)$ & $12(32,4)$ & $26(31,3)$ \\
\hline
\end{tabular}




\subsubsection{Anteil der Einsätze nach Rettungsmittel}

Mit 84,1\% wurde die Mehrzahl der Einsätze von den Teams des Rettungshubschraubers (RTH) durchgeführt. 13 Kinder (15,9\%) wurden durch die Notärzte der Notarzteinsatzfahrzeuge (NEF) intubiert.

Abbildung 8: Kreisdiagramm Rettungsmittel

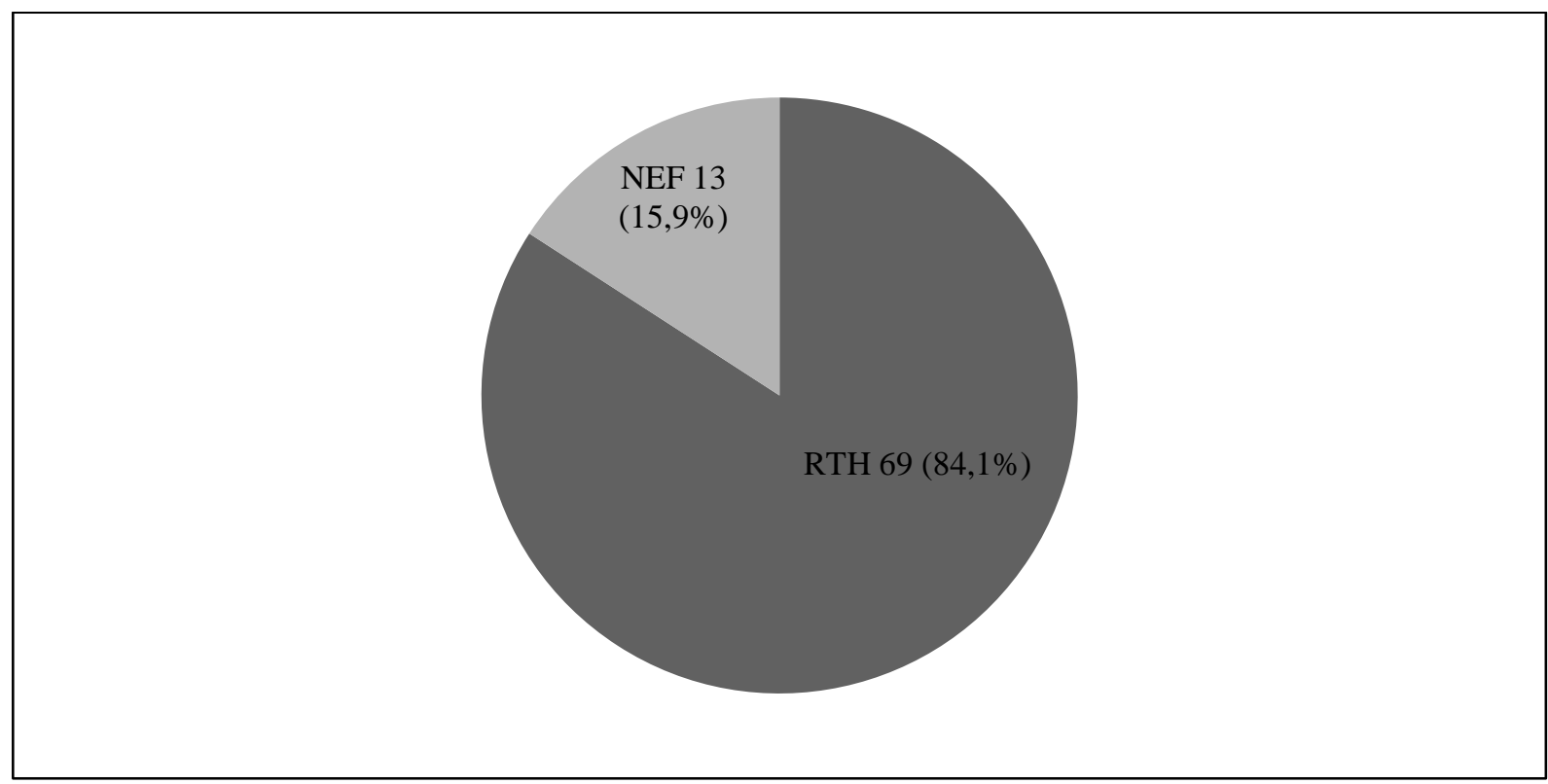

Tabelle 7: Art des Rettungsmittels nach Altersgruppen

\begin{tabular}{lllll}
\hline & $<$ 1 Jahr & 1-5 Jahre & 6-14 Jahre & Alle \\
& $\mathrm{n}=17(\%)$ & $\mathrm{n}=28(\%)$ & $\mathrm{n}=37(\%)$ & $\mathrm{n}=82(\%)$ \\
\hline RTH & $11(64,7)$ & $25(89,3)$ & $33(89,2)$ & $69(84,1)$ \\
NEF & $6(35,3)$ & $3(10,7)$ & $4(10,8)$ & $13(15,9)$ \\
\hline
\end{tabular}

Bezogen auf die Behandlungsgruppen ist von Bedeutung (Tabelle 8), dass in der Gruppe 2 alle Einsätze durch die Rettungshubschrauberbesatzungen versorgt wurden. In der Regel wurde der RTH in diesen Fällen zur Unterstützung eines bodengebundenen Notarztes alarmiert und ein luftgebundener Transport in ein geeignetes Zentrum durchgeführt.

Tabelle 8: Art des Rettungsmittels nach Behandlungsgruppen

\begin{tabular}{llll}
\hline & Gruppe 1 & Gruppe 2 & Alle \\
& $\mathrm{n}=58(\%)$ & $\mathrm{n}=24(\%)$ & $\mathrm{n}=82(\%)$ \\
\hline RTH & $45(77,6)$ & $24(100,0)$ & $69(84,1)$ \\
NEF & $13(22,4)$ & 0 & $13(15,9)$ \\
\hline
\end{tabular}




\subsubsection{Eintreffzeiten und präklinische Versorgungsintervalle}

Die Analyse der präklinischen Versorgungsintervalle beschränkt sich auf die Fälle der Gruppe 1. Die Zeiten der Gruppe 2 konnten nicht zuverlässig kalkuliert werden, da die Alarmierungszeiten der zuerst eintreffenden notarztbesetzten Rettungsmittel nicht exakt bekannt waren.

Die in den Abbildungen 9 und 10 dargestellten Boxplots entsprechen der 25. bis 75 . Perzentile (1. und 3. Quartil) mit Median. Die Whisker reichen von Minimum bis Maximum.

Der Median der Eintreffzeiten von Alarmierung bis zum Eintreffen der Rettungsmittel an der Einsatzstelle betrug 9,0 Minuten (Bereich 2-24; Mittelwert 9,6 \pm Standardabweichung 5,2).

Abbildung 9: Boxplot der Eintreffzeiten der Gruppe 1

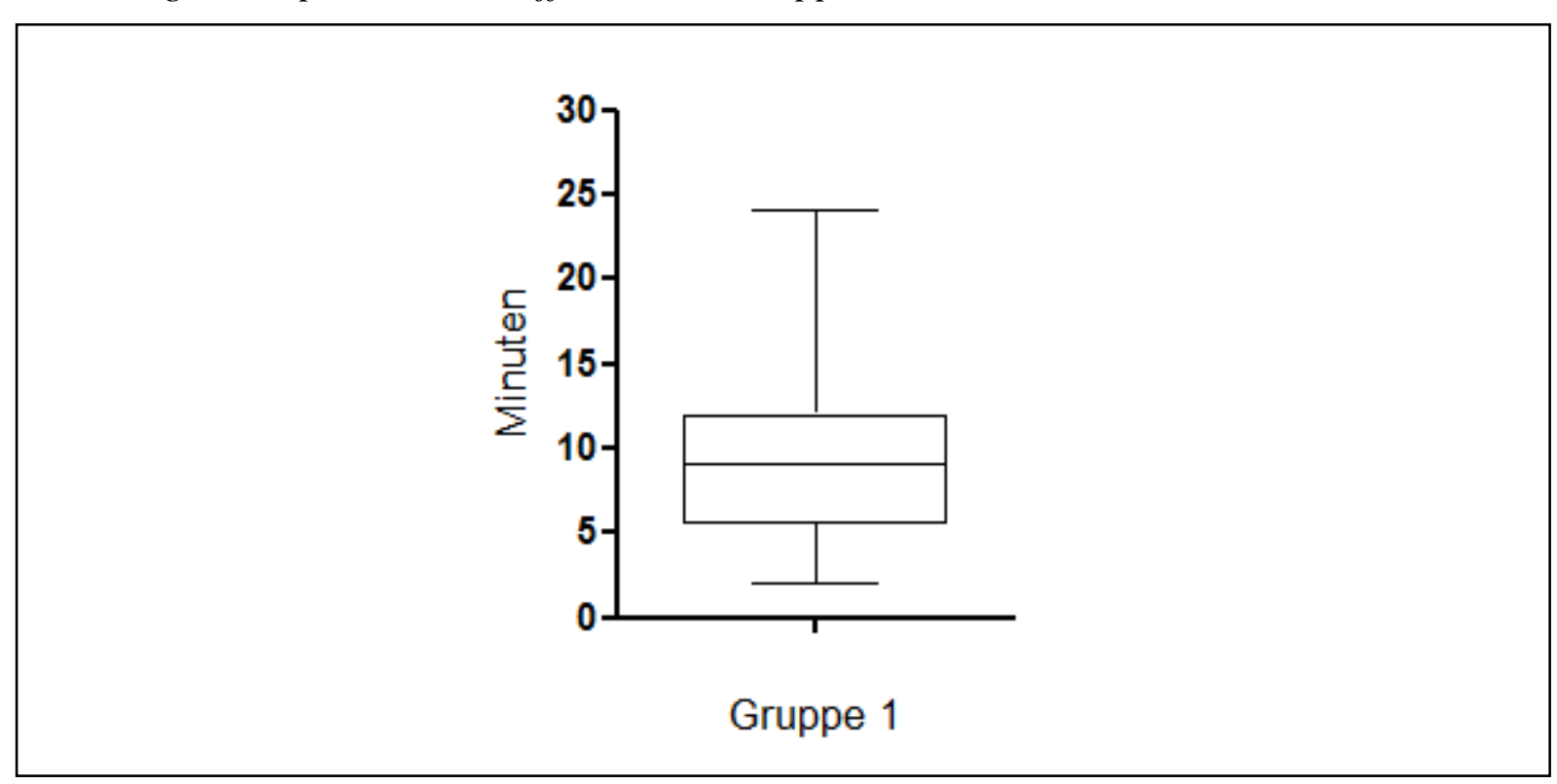


Abbildung 10 zeigt den Boxplot der Zeitintervalle von Alarmierung des Rettungsmittels bis Eintreffen in die Zielklinik (präklinisches Versorgungsintervall). Im Median wurden die Kinder 52 Minuten (Bereich 27-99) nach Alarmierung des Rettungsmittels in der definitiven Zielklinik übergeben (Mittelwert 54,6 \pm Standardabweichung 16,8).

Abbildung 10: Boxplot der präklinischen Versorgungsintervalle der Gruppe 1

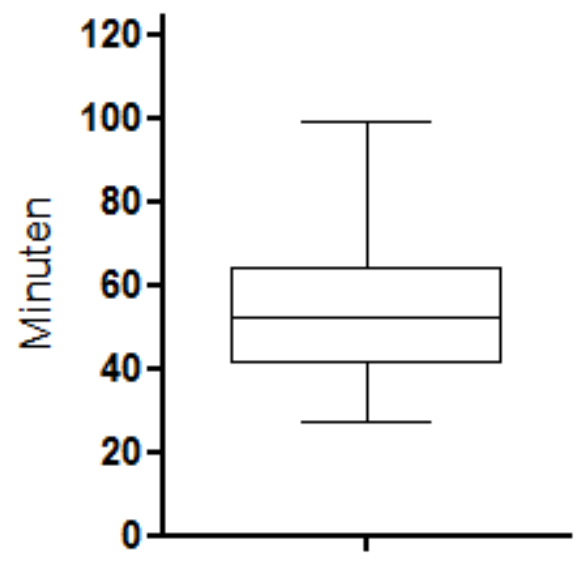

Gruppe 1 


\subsubsection{Zielkliniken}

Von 82 intubierten Kindern wurden $65(79,3 \%)$ in die Universitätsmedizin Göttingen transportiert. Zwölf Kinder (14,6\%) wurden in anderen Kliniken weiterversorgt. In all diesen Fällen fand der Transport mit dem Rettungshubschrauber in spezialisierte Einrichtungen wie Verbrennungszentren oder andere überregionale Traumazentren statt. Alle Zielkliniken waren Kliniken der Maximalversorgung oder Kliniken mit pädiatrischer Schwerpunktversorgung. Bei fünf Einsätzen wurde nach einer Todesfeststellung am Notfallort kein Transport durchgeführt.

\section{Abbildung 11: Kreisdiagramm Transportziele}

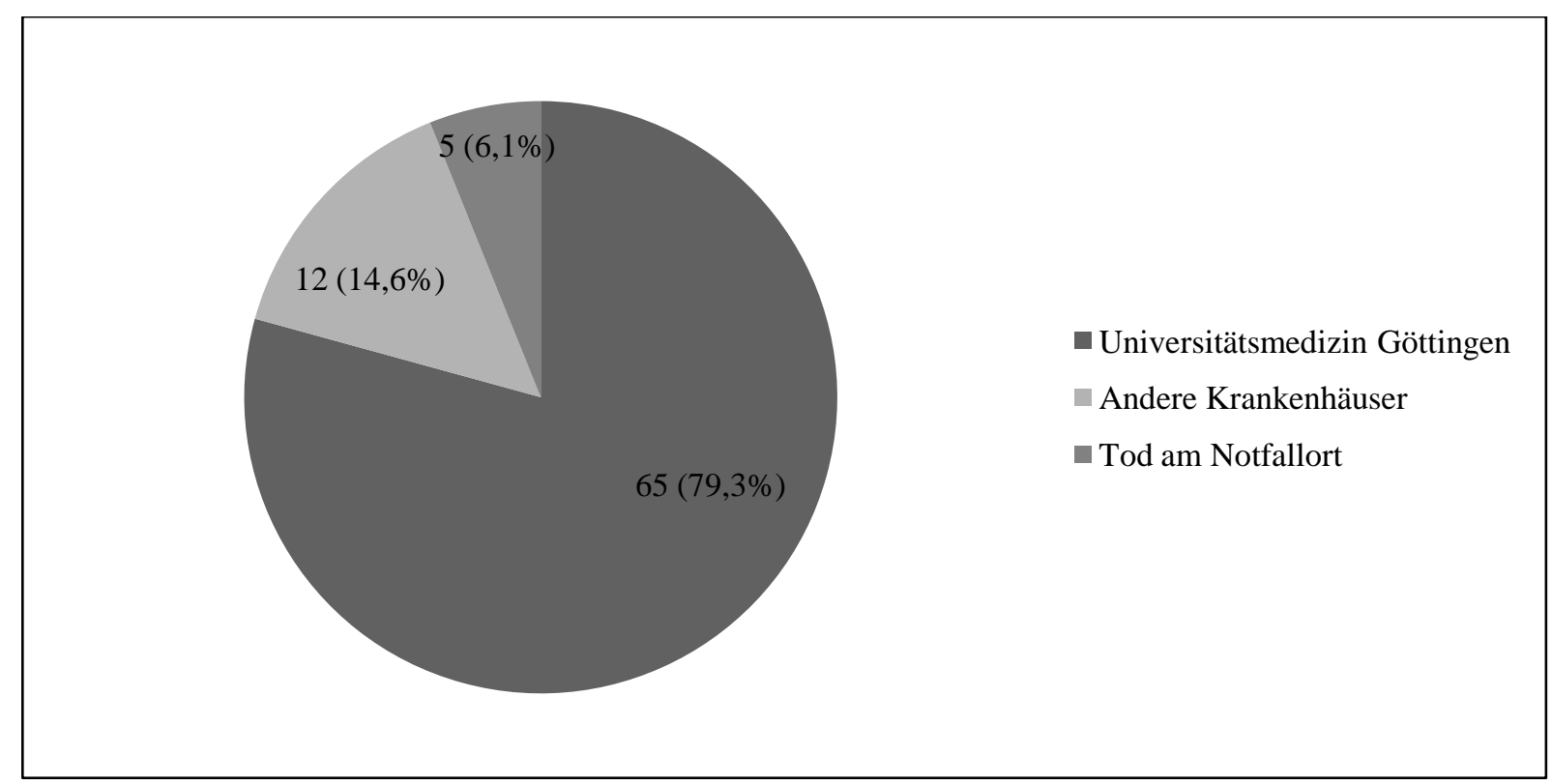




\subsection{Diagnosen}

Die weitaus häufigste Diagnose, die zur Intubation von Kindern führte, waren in der Hälfte aller Fälle Traumata. Krampfanfälle $(13,4 \%)$ und plötzlicher Kindstod $(12,2 \%)$ folgten in der Häufigkeit. Diese drei Diagnosegruppen ergaben mehr als dreiviertel aller Diagnosen.

\section{Abbildung 12: Notfalldiagnosen}

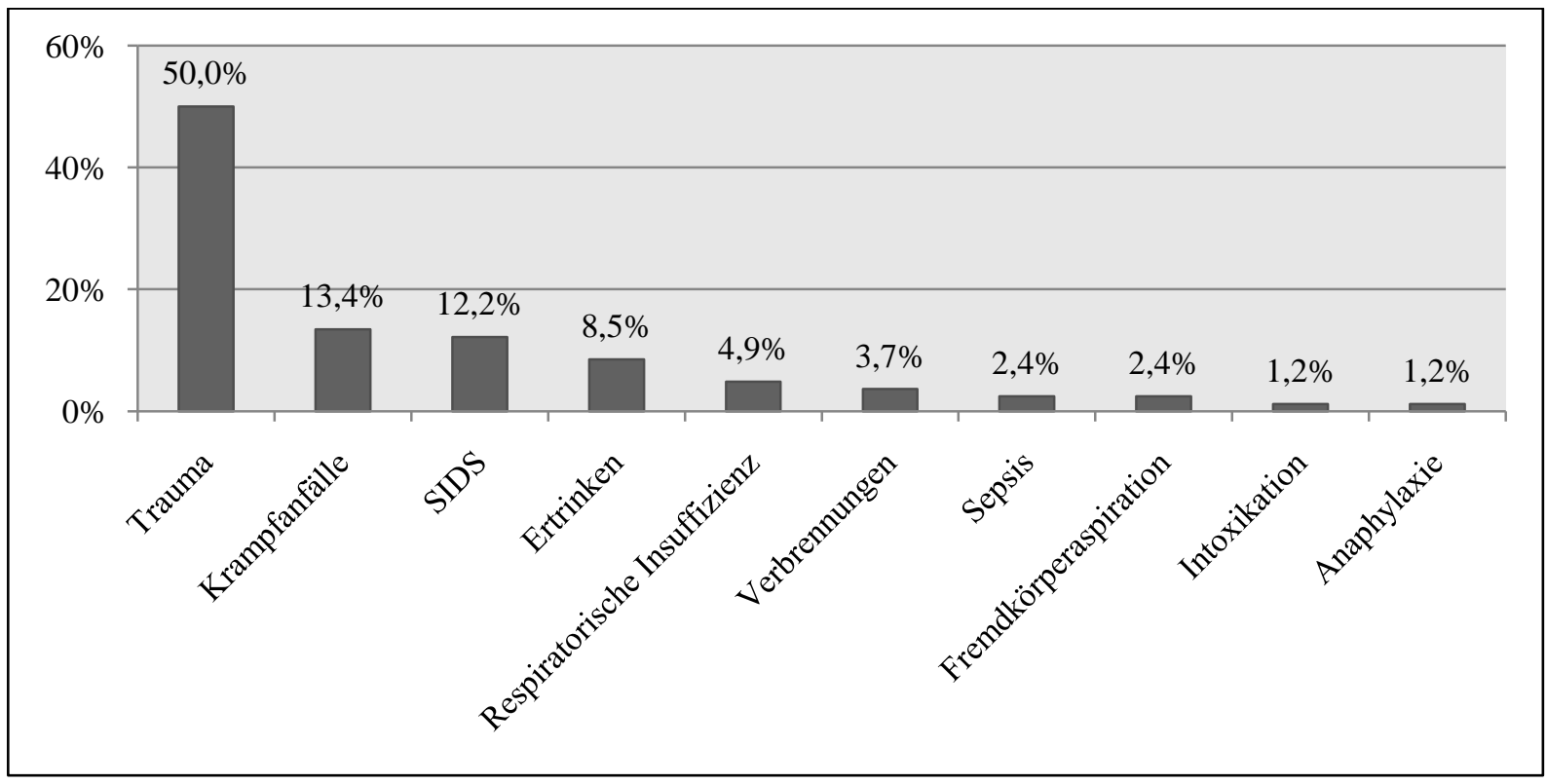

Die Häufigkeit der Diagnosen in Abhängigkeit von der Altersgruppe zeigt die altersabhängige Verteilung der Prävalenzen (Tabelle 9). In der Altersgruppe Schulkinder ist Trauma mit 73\% die dominierende Diagnose. Bei den Säuglingen stellte der plötzliche Kindstod (SIDS) die häufigste Diagnose dar, wohingegen bei den Kleinkindern Trauma und Krampfanfälle häufige Diagnosen waren, die eine ETI erforderlich machten.

Tabelle 9: Häufigkeit der Einweisungsdiagnosen in Abhängigkeit von der Altersgruppe

\begin{tabular}{|c|c|c|c|c|}
\hline Diagnosen & $\begin{array}{l}<1 \mathrm{Jahr} \\
\mathrm{n}=17(\%)\end{array}$ & $\begin{array}{l}\text { 1-5 Jahre } \\
\mathrm{n}=28(\%)\end{array}$ & $\begin{array}{l}\text { 6-14 Jahre } \\
\mathrm{n}=37(\%)\end{array}$ & $\begin{array}{l}\text { Alle } \\
\mathrm{n}=82(\%)\end{array}$ \\
\hline Trauma & $3(17,6)$ & $11(39,3)$ & $27(73,0)$ & $41(50,0)$ \\
\hline Krampfanfälle & $1(5,9)$ & $8(28,6)$ & $2(5,4)$ & $11(13,4)$ \\
\hline SIDS & $10(58,8)$ & 0 & 0 & $10(12,2)$ \\
\hline Ertrinken & 0 & $4(14,3)$ & $3(8,1)$ & $7(8,5)$ \\
\hline Respiratorische Insuffizienz & $1(5,9)$ & 0 & $3(8,1)$ & $4(4,9)$ \\
\hline Verbrennungen/Verbrühungen & $1(5,9)$ & $2(7,1)$ & 0 & $3(3,7)$ \\
\hline Sepsis & $1(5,9)$ & $1(3,6)$ & 0 & $2(2,4)$ \\
\hline Fremdkörperaspiration & 0 & $2(7,1)$ & 0 & $2(2,4)$ \\
\hline Intoxikation & 0 & 0 & $1(2,7)$ & $1(1,2)$ \\
\hline Anaphylaxie & 0 & 0 & $1(2,7)$ & $1(1,2)$ \\
\hline
\end{tabular}


In Tabelle 10 ist die Verteilung der Diagnosen nach Art des Rettungsmittels aufgeführt. Der Rettungshubschrauber wurde mit 55\% am häufigsten zu Notfällen mit traumabedingter präklinischer Intubation alarmiert, gefolgt von Krampfanfällen, SIDS und Ertrinken mit je 10\%. Die führenden Diagnosen, die bei NEF-versorgten Notfällen zur ETI eines Kindes veranlassten, waren Krampfanfälle (30\%), Trauma und SIDS (je 23\%) sowie respiratorische Notfälle (15\%).

Tabelle 10: Diagnosen nach Art des Rettungsmittels

\begin{tabular}{lll}
\hline & $\mathrm{RTH}$ & $\mathrm{NEF}$ \\
Diagnose & $\mathrm{n}=69(\%)$ & $\mathrm{n}=13(\%)$ \\
\hline Trauma & $38(55,1)$ & $3(23,1)$ \\
Krampfanfälle & $7(10,1)$ & $4(30,8)$ \\
SIDS & $7(10,1)$ & $3(23,1)$ \\
Ertrinken & $7(10,1)$ & 0 \\
Respiratorische Insuffizienz & $2(2,9)$ & $2(15,4)$ \\
Verbrennungen/Verbrühungen & $3(4,3)$ & 0 \\
Sepsis & $1(1,4)$ & $1(7,7)$ \\
Fremdkörperaspiration & $2(2,9)$ & 0 \\
Intoxikation & $1(1,4)$ & 0 \\
Anaphylaxie & $1(1,4)$ & 0 \\
\hline
\end{tabular}




\subsubsection{Schweregradeinschätzung anhand der NACA- und GCS-Scores}

80 Kinder (97,6\%) wurden den NACA-Scores IV-VII zugeordnet, also mit akuter

Vitalbedrohung oder bereits eingetretenem Tod. Im Median lag der NACA-Score bei V. Zwei Schulkinder mit Schweregradeinstufung NACA III wurden aufgrund eines isolierten Extremitätentraumas zur Durchführung einer effektiven Schmerztherapie vor Hubschraubertransport semi-elektiv intubiert.

Abbildung 13: Verteilung der NACA-Scores

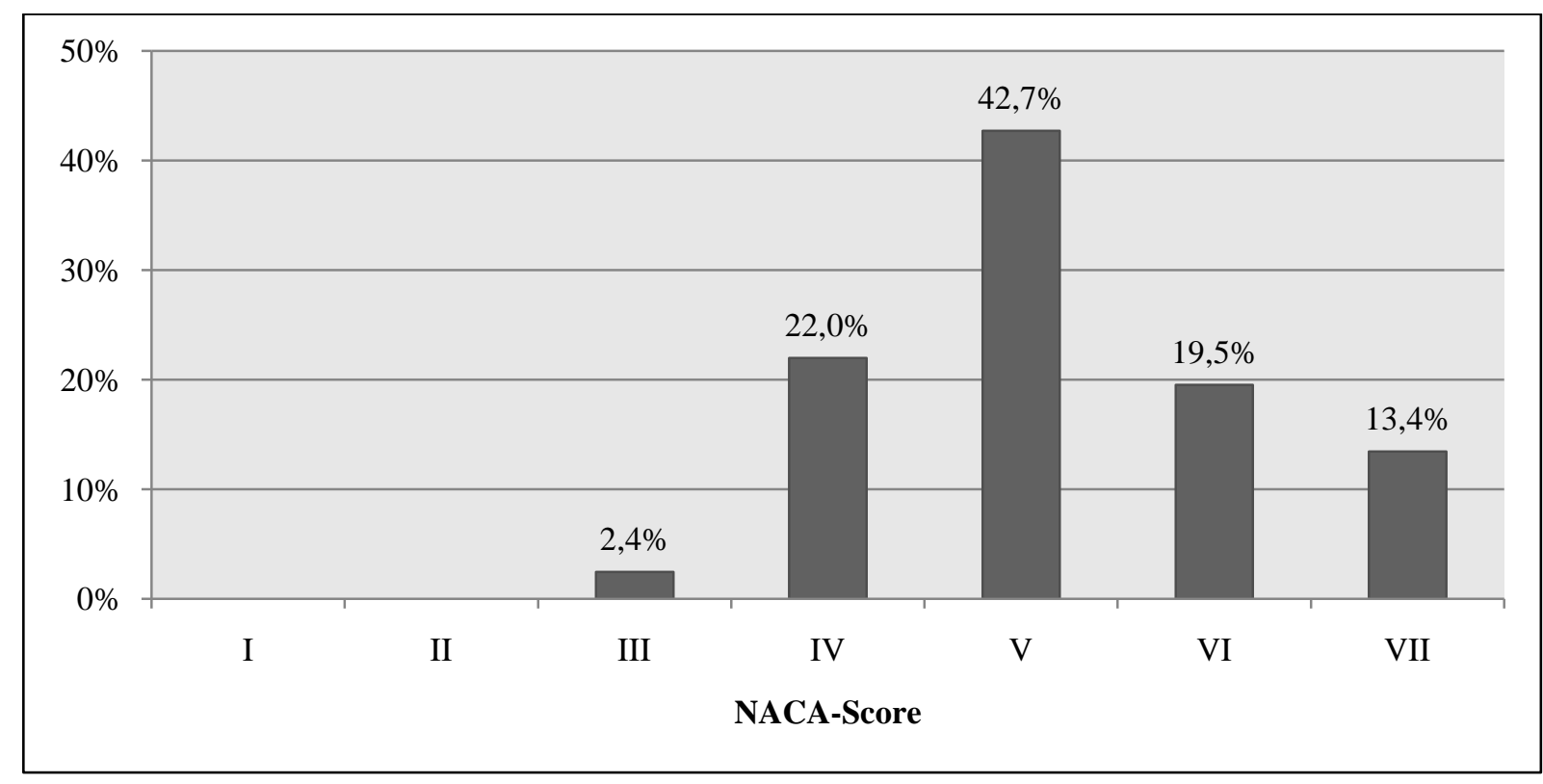

Tabelle 11: Verteilung der NACA-Scores nach Altersgruppen

\begin{tabular}{cllll}
\hline & $<1 \mathrm{Jahr}$ & 1-5 Jahre & 6-14 Jahre & Alle \\
NACA & $\mathrm{n}=17(\%)$ & $\mathrm{n}=28(\%)$ & $\mathrm{n}=37(\%)$ & $\mathrm{n}=82(\%)$ \\
\hline I & 0 & 0 & 0 & 0 \\
II & 0 & 0 & 0 & 0 \\
III & 0 & 0 & $2(5,4)$ & $2(2,4)$ \\
IV & $1(5,9)$ & $6(21,4)$ & $11(29,7)$ & $18(22,0)$ \\
V & $4(23,5)$ & $15(53,6)$ & $16(43,2)$ & $35(42,7)$ \\
VI & $3(17,6)$ & $6(21,4)$ & $7(18,9)$ & $16(19,5)$ \\
VII & $9(52,9)$ & $1(3,6)$ & $1(2,7)$ & $11(13,4)$ \\
\hline
\end{tabular}


Die histographische Verteilung der Glasgow-Coma-Scale (GCS)-Werte ist in Abbildung 14 dargestellt. 76,9\% zeigten einen GCS $\leq 8$ Punkte, 48,8\% waren komatös (GCS 3). 23,1\% der Studienpopulation wiesen einen GCS von $\geq 8$ Punkten und 14,6\% zeigten mit einem GCS von 15 Punkten keine neurologischen Defizite. Ein Teil dieser Patienten wurden am Notfallort semi-elektiv u.a. zum Lufttransport endotracheal intubiert. Der GCS betrug im Median 4 Punkte.

Abbildung 14: Verteilung der Glasgow-Coma-Scale-Werte

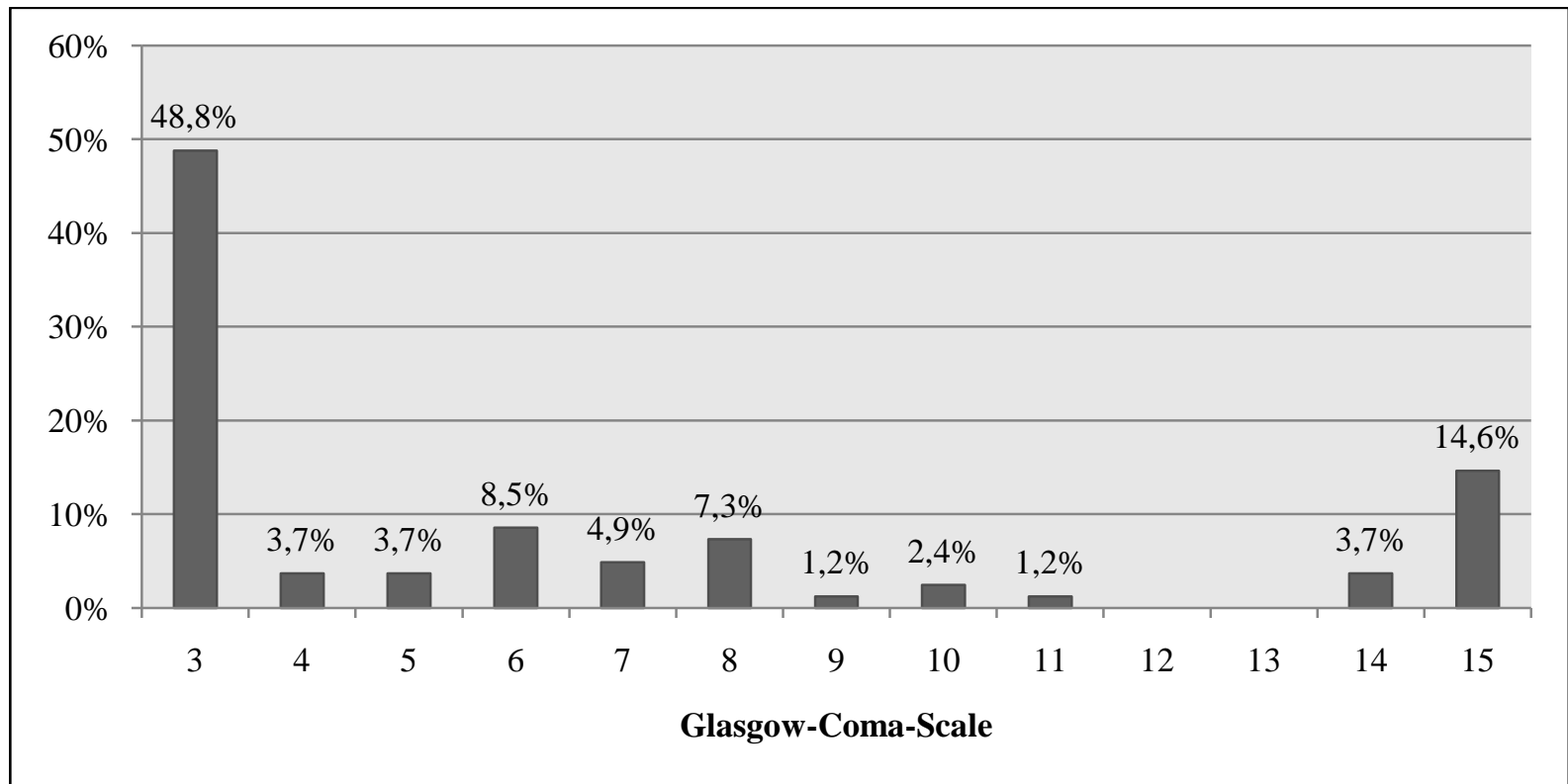




\subsection{Atemwegscharakteristika}

Die Atemwegsinterventionen wurden nur in den 58 Fällen dokumentiert, die der Behandlungsgruppe 1 (Intubation durch Studienärzte) zugeordnet wurden.

\subsubsection{Cormack-Lehane-Scores}

Bei der direkten Laryngoskopie wurde bei neun Kindern (15,5\%) ein Cormack-Lehane-Score $\geq 3$ vergeben, welcher ein valider Prädiktor für eine schwierige Intubation ist. Säuglinge hatten mit $23,1 \%$ eine höhere Inzidenz von Cormack-Lehane-Scores $\geq 3$ als die anderen beiden Altersgruppen. Der Unterschied ist jedoch nicht statistisch signifikant (Spearman'sRho-Test: $p>0.70)$.

Abbildung 15: Verteilung der Cormack-Lehane-Scores

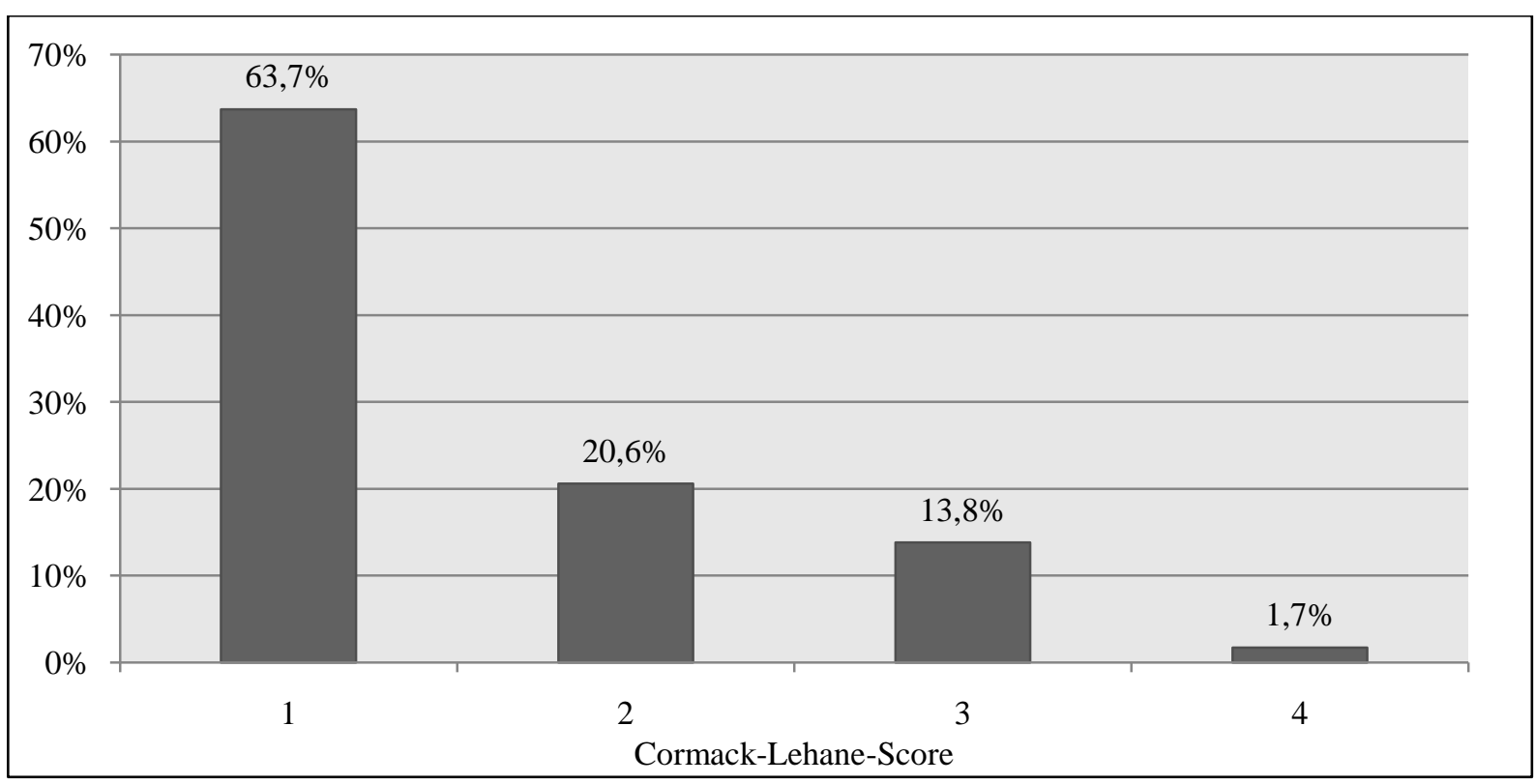

Tabelle 12: Evaluierung der Cormack-Lehane-Scores durch die Studienärzte (Gruppe 1)

\begin{tabular}{clllll}
\hline & $<$ 1 Jahr & 1-5 Jahre & 6-14 Jahre & Alle & \\
Cormack-Lehane & $\mathrm{n}=13(\%)$ & $\mathrm{n}=22(\%)$ & $\mathrm{n}=23(\%)$ & $\mathrm{n}=58(\%)$ & $\mathrm{p}$-Wert \\
\hline 1 & $7(53,8)$ & $16(72,7)$ & $14(60,9)$ & $37(63,7)$ & \\
2 & $3(23,1)$ & $3(13,6)$ & $6(26,1)$ & $12(20,6)$ & \\
3 & $2(15,4)$ & $3(13,6)$ & $3(13,0)$ & $8(13,8)$ & für CL $\geq 3:$ \\
4 & $1(7,7)$ & 0 & 0 & $1(1,7)$ & $\mathrm{p}>0,70$ \\
\hline
\end{tabular}




\subsubsection{Inzidenz und Ursachen einer schwierigen Intubation}

Die ETI wurde in $13,8 \%$ aller Fälle durch die Studienärzte als schwierig eingestuft. In der Altersgruppe der Säuglinge war die Inzidenz einer schwierigen Intubation mit 23,1\% wesentlich häufiger, wenngleich auch nicht statistisch signifikant ( $p>0.25)$.

Abbildung 16: Inzidenz der schwierigen Intubation

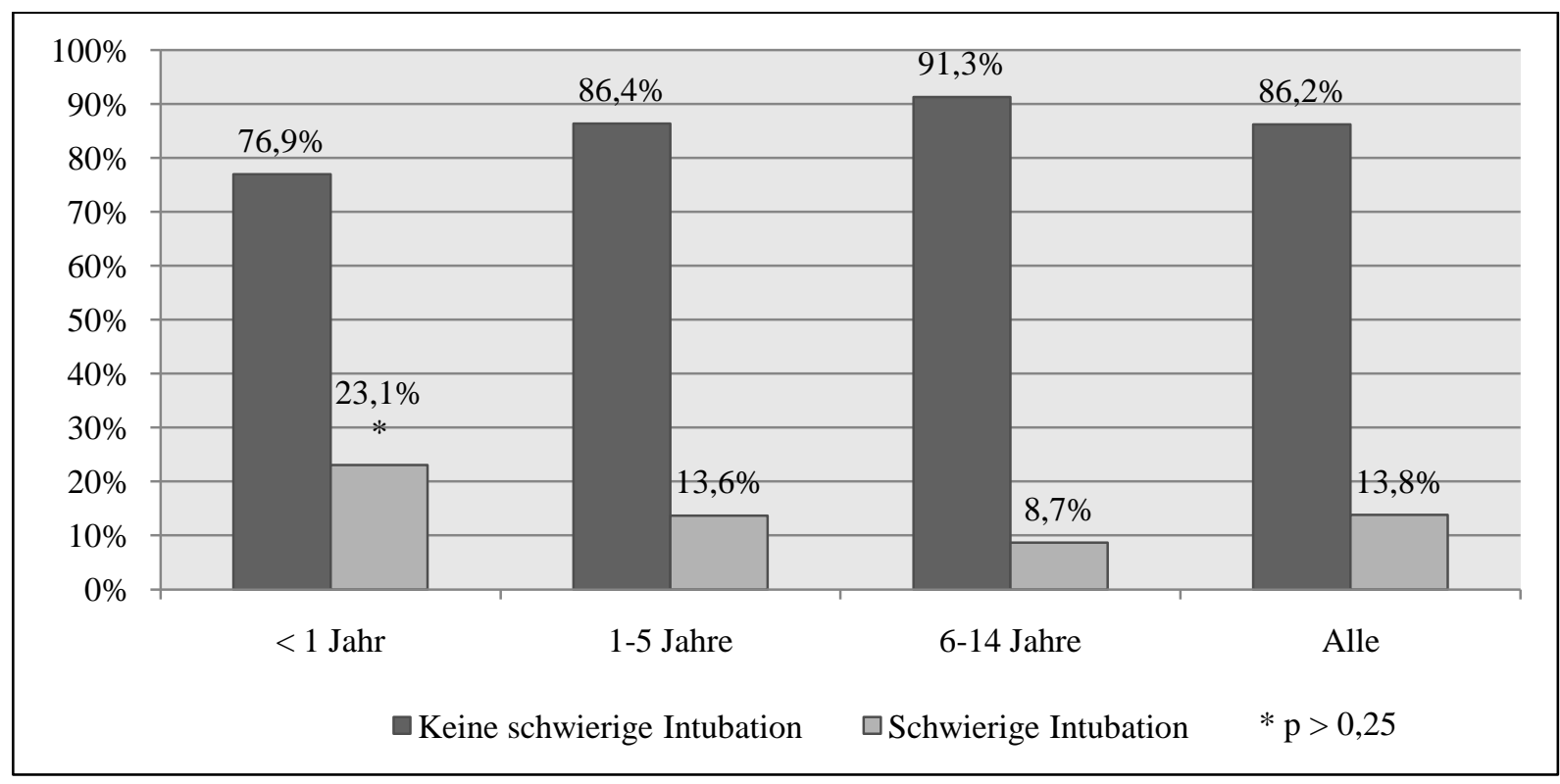

Tabelle 13: Inzidenz einer schwierigen Intubation (Gruppe 1)

\begin{tabular}{llllll}
\hline & $<1 \mathrm{Jahr}$ & 1-5 Jahre & 6-14 Jahre & Alle & \\
& $\mathrm{n}=13(\%)$ & $\mathrm{n}=22(\%)$ & $\mathrm{n}=23(\%)$ & $\mathrm{n}=58(\%)$ & $\mathrm{p}$-Wert \\
\hline Keine schwierige Intubation & $10(76.9)$ & $19(86.3)$ & $21(91.3)$ & $50(86.2)$ & \\
Schwierige Intubation & $3(23.1)$ & $3(13.6)$ & $2(8.7)$ & $8(13.8)$ & $\mathrm{p}>0.25$ \\
\hline
\end{tabular}

Wenn Intubationsschwierigkeiten dokumentiert waren, wurden sie in der Mehrzahl der Fälle anatomischen Ursachen zugeschrieben (Tabelle 14; Mehrfachnennungen waren möglich).

Tabelle 14: Ursachen einer schwierigen Intubation

\begin{tabular}{lcccc}
\hline Ursache & $\begin{array}{c}\text { 1 Jahr } \\
\mathrm{n}=3\end{array}$ & $\begin{array}{c}1-5 \text { Jahre } \\
\mathrm{n}=3\end{array}$ & $\begin{array}{c}\text { 6-14 Jahre } \\
\mathrm{n}=2\end{array}$ & $\begin{array}{c}\text { Alle } \\
\mathrm{n}=8\end{array}$ \\
\hline Anatomisch & 3 & 1 & 2 & 6 \\
Große Zunge & 2 & 0 & 1 & 3 \\
Reduzierte Mundöffnung & 0 & 0 & 1 & 1 \\
Andere & 1 & 1 & 0 & 2 \\
Trauma & 0 & 0 & 0 & 0 \\
Position & 1 & 1 & 0 & 2 \\
Andere & 1 & 1 & 0 & 2 \\
\hline
\end{tabular}




\subsubsection{Erfolgsraten der endotrachealen Intubation}

Von 82 Kindern, bei denen ein präklinischer Intubationsversuch unternommen wurde, konnten 81 Kinder erfolgreich intubiert werden. Bei einem Säugling in der Behandlungsgruppe 1 misslang die endotracheale Intubation nach multiplen Versuchen, bevor schließlich eine Larynxmaske platziert wurde. Die Erfolgsrate beider Gruppen betrug somit 98,8\%. 52 Kinder wurden orotracheal und sechs Kinder nasotracheal intubiert (jeweils drei Säuglinge und drei Kleinkinder).

Die kumulative Erfolgsrate in der Gruppe 1 lag bei 98,3\%. Die Analyse der Erfolgsraten in Abhängigkeit von der Anzahl der Intubationsversuche ergab, dass 84,5\% aller Intubationen im 1. Versuch erfolgreich waren.

\section{Abbildung 17: Kumulative Erfolgsrate (Gruppe 1)}

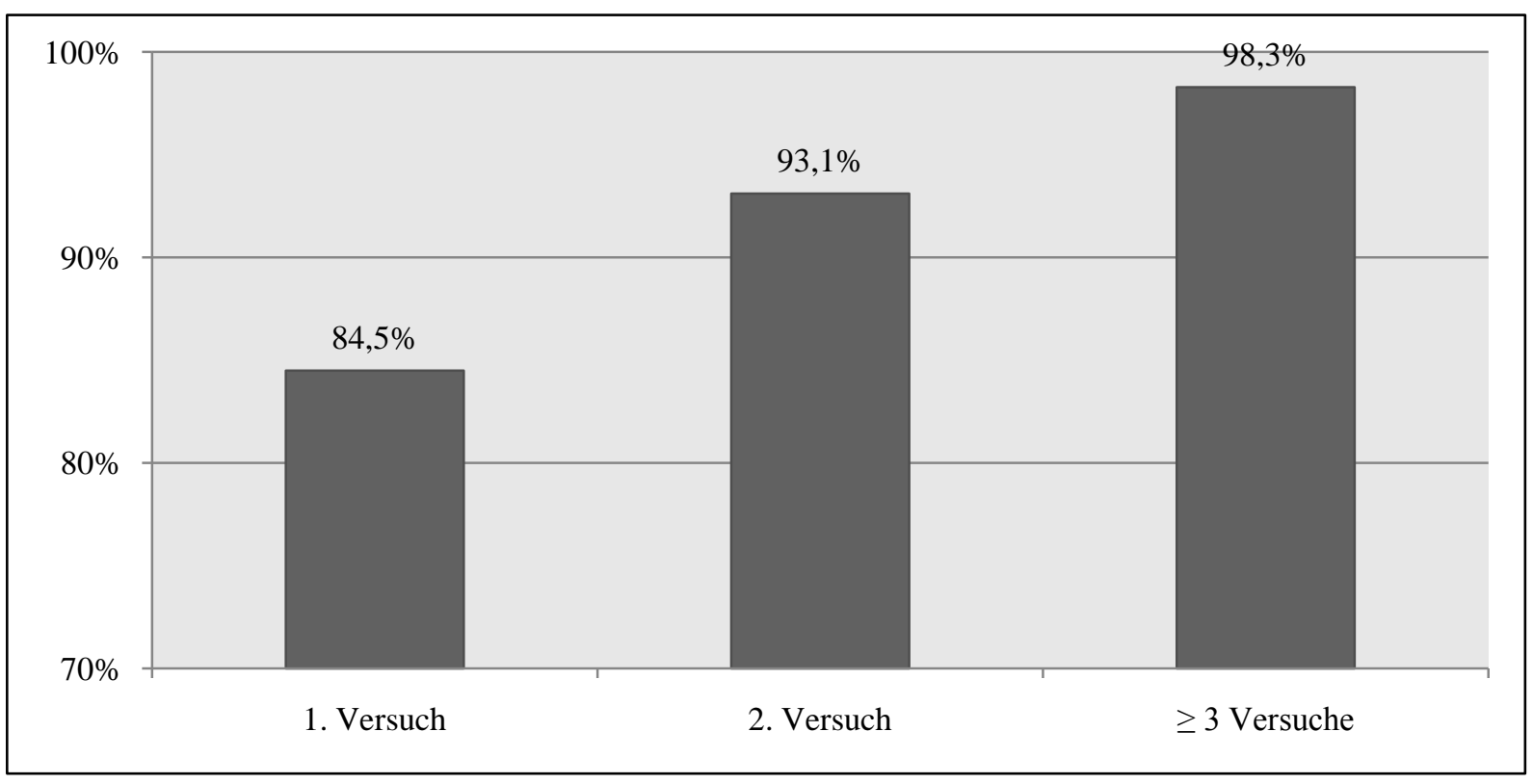


In Abbildung 18 sind die Erfolgsraten der Intubationsversuche nach Altersgruppen histographisch dargestellt. In der Altersgruppe Schulkinder waren die Erfolgsquoten mit 95,7\% im ersten Versuch am höchsten. Kleinkinder und Säuglinge hatten mit je $77 \%$ geringere Erfolgsraten im ersten Intubationsversuch (Spearman's-Rho-Test: $p=0,04$ ).

Abbildung 18: Erfolgsraten der Intubationsversuche (Gruppe 1, $n=58$ )

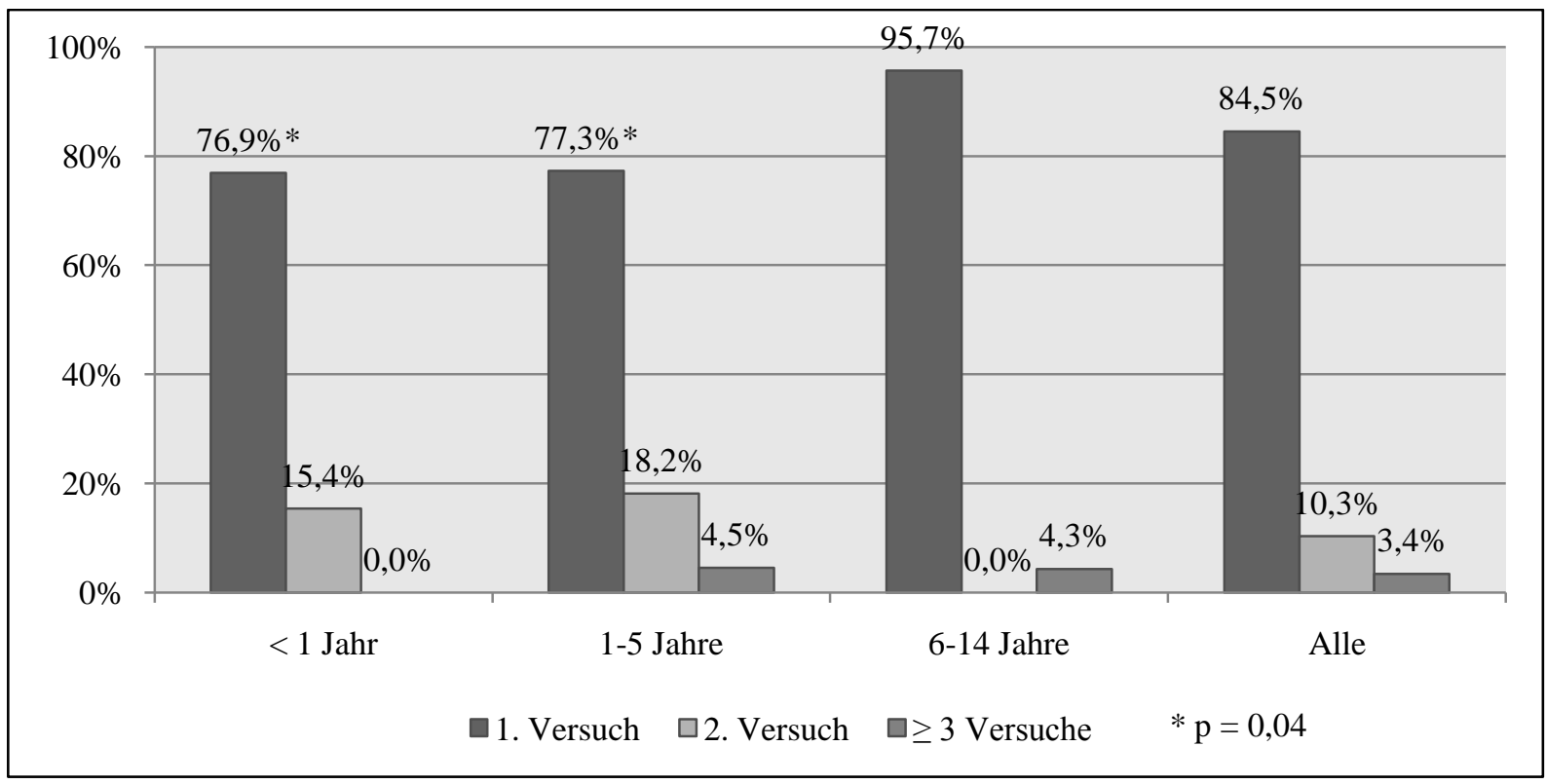

In Tabelle 15 ist die Anzahl der Intubationsversuche in Abhängigkeit von den Altersgruppen dargestellt.

Tabelle 15: Analyse der Intubationsversuche in der Gruppe 1

\begin{tabular}{cclllll}
\hline & & $<$ Jahr & 1-5 Jahre & 6-14 Jahre & Alle & \\
Route & Erfolgsrate & $\mathrm{n}=13(\%)$ & $\mathrm{n}=22(\%)$ & $\mathrm{n}=23(\%)$ & $\mathrm{n}=58(\%)$ & $\mathrm{p}$-Wert \\
\hline Oral & 1. Versuch & $7(53,9)$ & $15(68,2)$ & $22(95,7)$ & $44(75,9)$ & $\mathrm{p}=0.04$ \\
$\mathrm{n}=52$ & 2. Versuch & $2(15,4)$ & $3(13,6)$ & 0 & $5(8,7)$ & \\
& $\geq 3$ Versuche & 0 & $1(4,5)^{\mathrm{a}}$ & $1(4,4)$ & $2(3,4)$ & \\
& ETI nicht erfolgreich & $1(7,7){ }^{\mathrm{b}}$ & 0 & 0 & $1(1,7)$ & \\
\hline Nasal & 1. Versuch & $3(23,1)$ & $2(9,1)$ & 0 & $5(8,6)$ & \\
$\mathrm{n}=6$ & 2. Versuch & 0 & $1(4,5)$ & 0 & $1(1,7)$ & \\
& $\geq 3$ Versuche & 0 & 0 & 0 & 0 & \\
\hline
\end{tabular}

In einem Fall bei einem Kleinkind misslang die Intubation durch den ersten Notarzt nach drei Versuchen und war schließlich nach insgesamt fünf Versuchen von einem zweiten, zur Unterstützung angeforderten Notarzt, erfolgreich $\left({ }^{\mathrm{a}}\right)$. In einem anderen Fall bei einem Säugling war die Intubation auch nach mehreren Versuchen nicht erfolgreich und das Kind konnte mit einer Larynxmaske ventiliert und oxygeniert werden $\left({ }^{b}\right)$. 


\subsubsection{Intubationszwischenfälle}

Intubationszwischenfälle (siehe 2.6.1) traten in 10 von 82 Fällen auf (12,2\%). Wie aus Abbildung 19 hervorgeht, war die Häufigkeit dieser Komplikationen höher in der Gruppe 2 $(6 / 24,25,9 \%)$ im Vergleich zur Gruppe 1 (4/58, 6,9\%). Die Signifikanztestung im Exakten Fisher-Test zur Korrelation der beiden Behandlungsgruppen ergab einen p-Wert von 0,06 und verfehlte knapp die statistische Signifikanz.

\section{Abbildung 19: Häufigkeit der Intubationszwischenfälle}

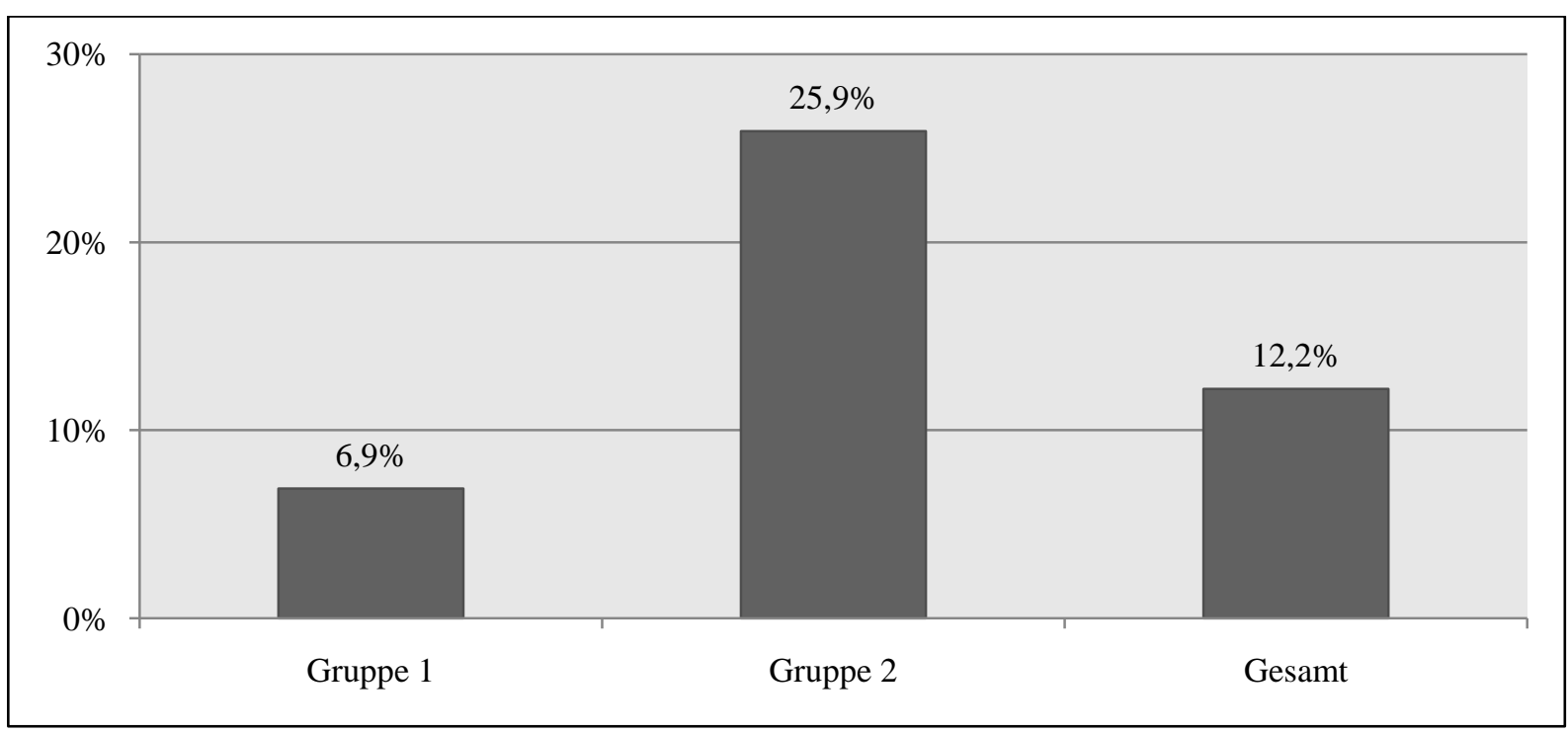

In Abbildung 20 ist die Häufigkeit der drei analysierten Intubationszwischenfälle dargestellt. In 4 Fällen wurde eine Tubusfehllage bzw. -Dislokation, in jeweils drei Fällen multiple Intubationsversuche oder misslungene Intubationen dokumentiert.

Abbildung 20: Arten der Intubationszwischenfälle

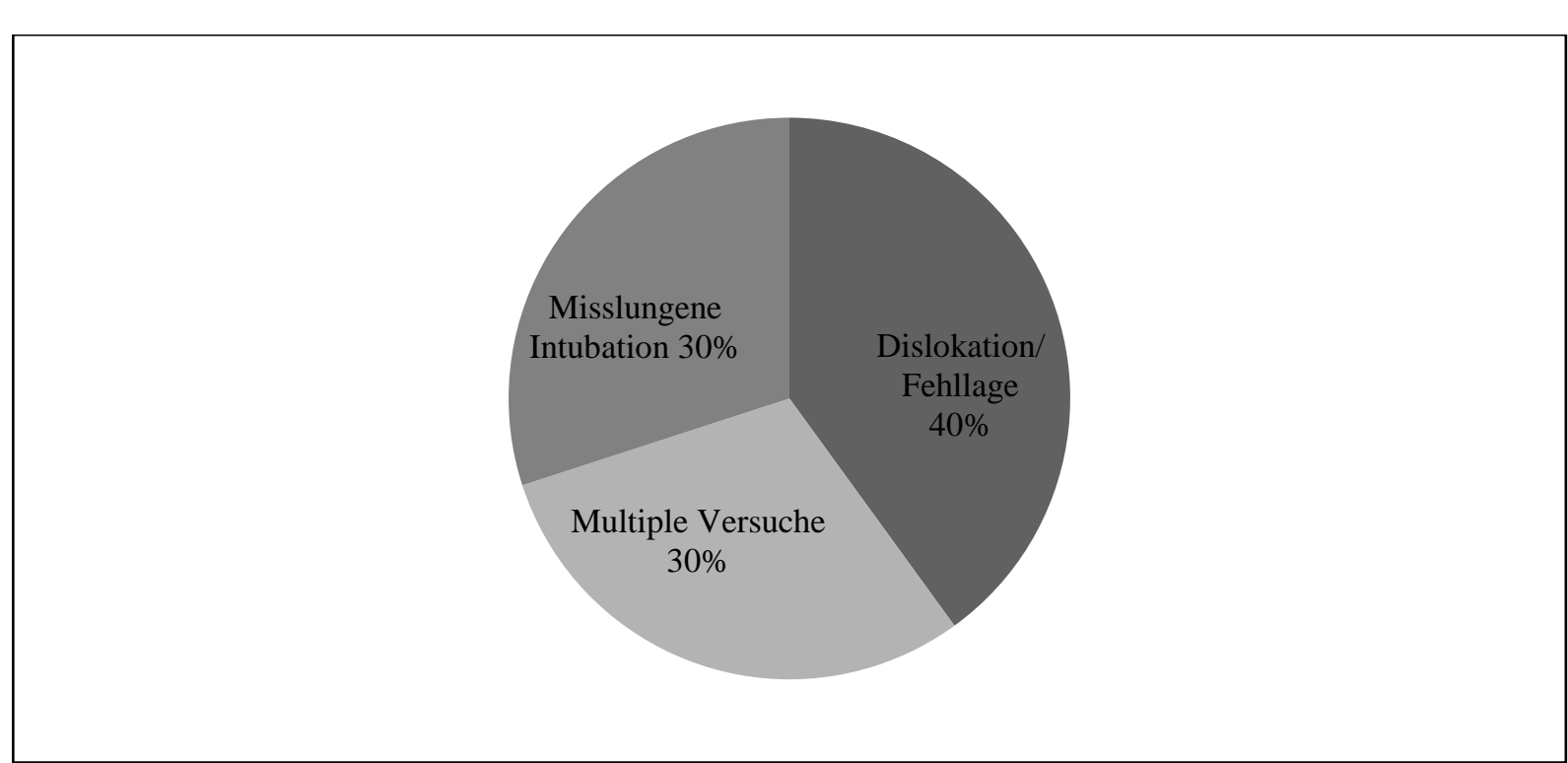




\subsection{Outcome}

71 von 82 Kindern $(86,6 \%)$ überlebten bis zur Krankenhausaufnahme und 63 Kinder (76,8\%) bis zur Entlassung aus dem Krankenhaus. 51 Kinder wurden nach Hause entlassen. Insgesamt verstarben 19 Kinder, darunter 10 Säuglinge (58,8\%). Die Überlebensraten der älteren Kinder waren signifikant besser als die der Säuglinge (Spearman's-Rho-Test)

Tabelle 16: Überlebensrate und Outcome in Korrelation zu den drei Altersgruppen

\begin{tabular}{llllll}
\hline & $<1$ Jahre & 1-5 Jahre & 6-14 Jahre & Alle & \\
& $\mathrm{n}=17(\%)$ & $\mathrm{n}=28(\%)$ & $\mathrm{n}=37(\%)$ & $\mathrm{n}=82(\%)$ & $\mathrm{p}$-Wert \\
\hline Überleben bis Klinik-Aufnahme & $8(47,1)$ & $27(96,4)$ & $36(97,3)$ & $71(86,6)$ & $\mathrm{p}<0,001$ \\
Überleben bei Klinik-Entlassung & $7(41,2)$ & $25(89,3)$ & $31(83,8)$ & $63(76,8)$ & $\mathrm{p}=0,01$ \\
Entlassen nach Hause & $3(42,9)$ & $21(84,0)$ & $27(87,1)$ & $51(81,0)$ & \\
Entlassen in Reha-Einrichtung & $4(57,1)$ & $4(16,0)$ & $4(12,9)$ & $12(19,0)$ & \\
Tod 12 Monate nach Ereignis & $10(58,8)$ & $3(10,7)$ & $6(16,2)$ & $19(23,2)$ & \\
Tod am Notfallort/Aufnahme & $9(90,0)$ & $1(33,3)$ & $1(16,7)$ & $11(57,9)$ & \\
Tod <24 h nach Ereignis & $1(10,0)$ & $1(33,3)$ & 0 & $2(10,5)$ & \\
Tod $>24$ nach Ereignis & 0 & $1(33,3)$ & $5(83,3)$ & $6(31,6)$ & \\
\hline
\end{tabular}

Tabelle 17 gibt einen vergleichenden Überblick über das Outcome in Korrelation zu den beiden Behandlungsgruppen. Die Überlebensrate bis zur Krankenhausentlassung (primäres Outcome) betrug $81 \%$ in der Gruppe 1 und 66,7\% in der Gruppe 2. Der Unterschied ist statistisch nicht signifikant (Exakter Fisher-Test: $\mathrm{p}=0,25$ ).

Tabelle 17: Überlebensrate und Outcome nach Behandlungsgruppen

\begin{tabular}{lllll}
\hline & Gruppe 1 & Gruppe 2 & Alle & \\
& $\mathrm{n}=58(\%)$ & $\mathrm{n}=24(\%)$ & $\mathrm{n}=82(\%)$ & $\mathrm{p}$-Wert \\
\hline Überleben bis Klinik-Aufnahme & $51(87,9)$ & $20(83,3)$ & $71(86,6)$ & $\mathrm{p}=0,72$ \\
Überleben bis Klinik-Entlassung & $47(81,0)$ & $16(66,7)$ & $63(76,8)$ & $\mathrm{p}=0,25$ \\
Entlassen nach Hause & $36(76,6)$ & $15(93,8)$ & $51(81,0)$ & \\
Entlassen in Reha-Einrichtung & $11(23,4)$ & $1(6,3)$ & $12(19,0)$ & \\
Tod 12 Monate nach Ereignis & $11(19,0)$ & $8(33,3)$ & $19(23,2)$ & \\
Tod am Notfallort/Aufnahme & $6(54,5)$ & $5(62,5)$ & $11(57,9)$ & \\
Tod < 24 h nach Ereignis & $2(18,2)$ & 0 & $2(10,5)$ & \\
Tod $>$ 24 h nach Ereignis & $3(27,3)$ & $3(37,5)$ & $6(31,6)$ & \\
\hline
\end{tabular}




\subsubsection{Dauer von Beatmung, Intensiv- und Krankenhausbehandlung}

Die dargestellten Boxplots in den Abbildungen 21, 22 und 23 entsprechen der 25. bis 75. Perzentile (1. und 3. Quartil) mit Median. Die Whisker reichen von Minimum bis Maximum.

Säuglinge waren im Median 7 Tage endotracheal intubiert und beatmet und damit deutlich länger als die Altersgruppen Klein- und Schulkinder (Median 1 Tag).

Abbildung 21: Boxplot der Beatmungsdauer nach Altersgruppen

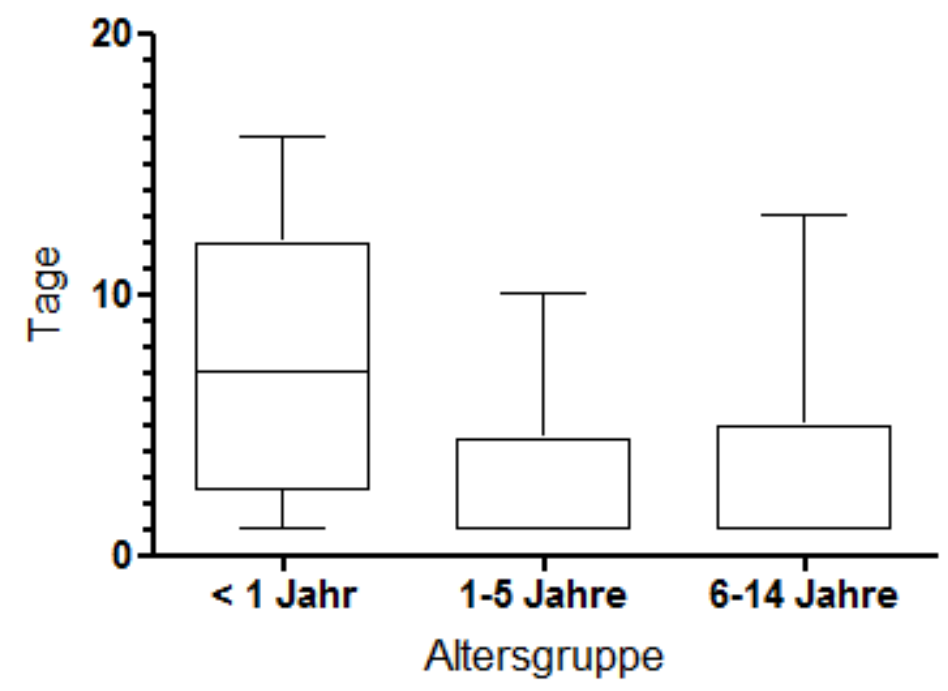

Auch hinsichtlich der Verweildauern auf der Intensivstation und im Krankenhaus hatten die Säuglinge im Median die längsten Aufenthalte im Vergleich zu den anderen Altersgruppen.

Abbildung 22: Boxplot der Intensivbehandlungsdauer nach Altersgruppen

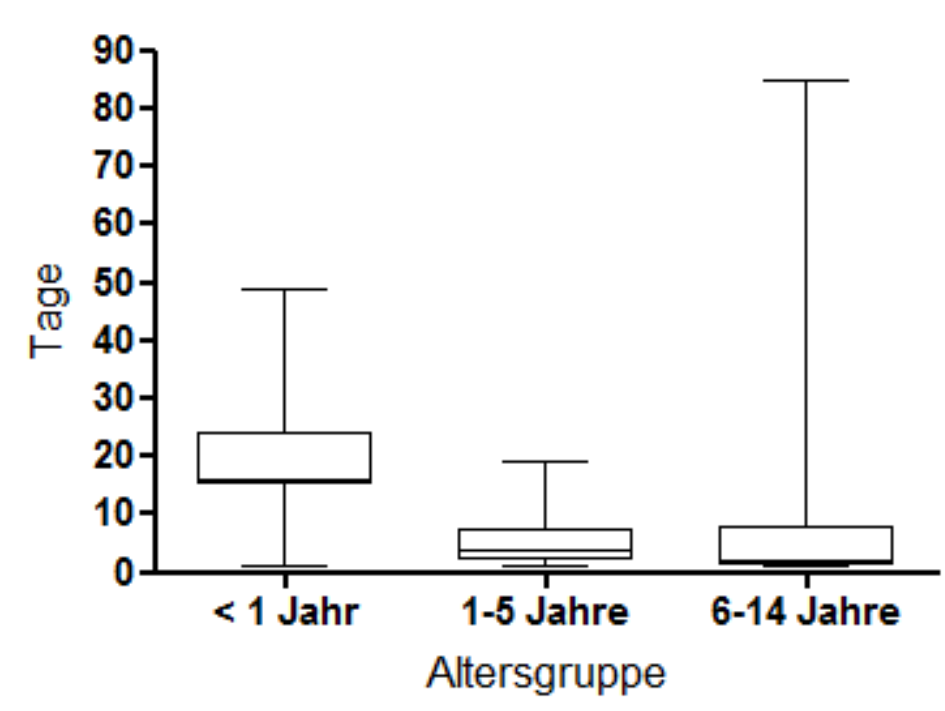


Abbildung 23: Boxplot der Krankenhausaufenthaltsdauer nach Altersgruppen

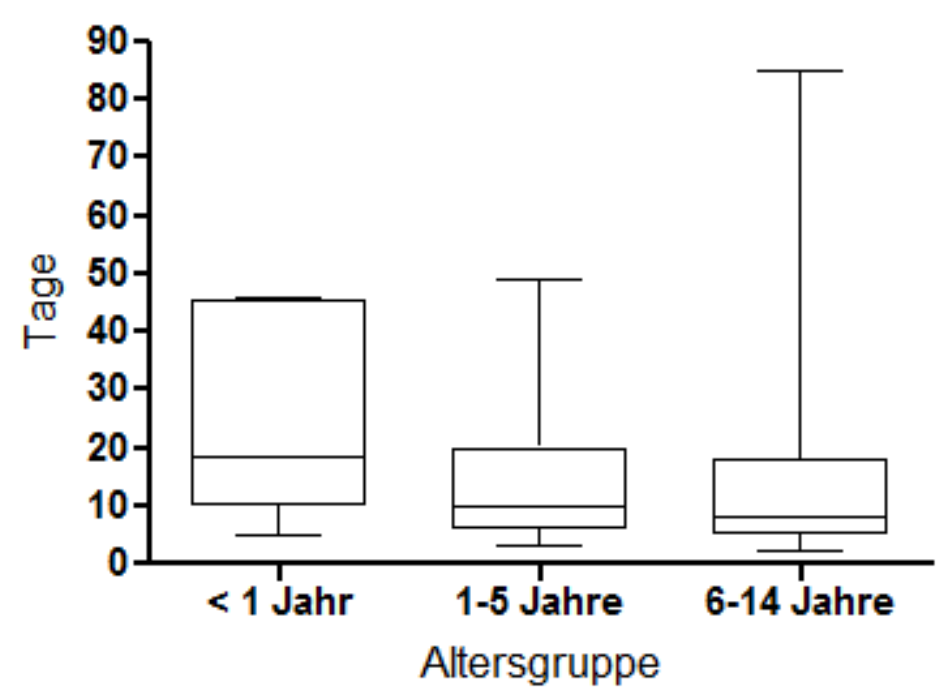




\subsubsection{Neurologisches Outcome bei Krankenhausentlassung}

54 Kinder zeigten ein gutes neurologisches Outcome zum Zeitpunkt der Entlassung aus dem Krankenhaus. Das neurologische Outcome war bei den älteren Kindern signifikant besser als bei den Säuglingen (Spearman's-Rho-Test: $\mathrm{p}<0,001)$.

Tabelle 18: Paediatric Cerebral Perfomance Categories (PCPC)

\begin{tabular}{llllll}
\hline & $<$ 1 Jahr & 1-5 Jahre & 6-14 Jahre & Alle \\
& $\mathrm{n}=17(\%)$ & $\mathrm{n}=28(\%)$ & $\mathrm{n}=37(\%)$ & $\mathrm{n}=82(\%)$ & $\mathrm{p}$-Wert \\
\hline PCPC 1 & $3(42,9)$ & $11(44,0)$ & $24(77,4)$ & $38(60,3)$ & \\
PCPC 2 & $0(0,0)$ & $4(16,0)$ & 0 & $4(6,3)$ & \\
PCPC 3 & $0(0,0)$ & $4(16,0)$ & 0 & $4(6,3)$ & \\
PCPC 4 & $3(42,9)$ & $5(20,0)$ & $6(19,4)$ & $14(22,2)$ & \\
PCPC 5 & $1(14,3)$ & $1(4,0)$ & $1(3,2)$ & $3(4,8)$ & \\
Delta-Score =0 & $0(0,0)$ & $6(25,0)$ & $5(16,1)$ & $11(17,5)$ & \\
Gutes neurologisches Outcome & $3(42,9)$ & $22(88,0)$ & $29(93,5)$ & $54(85,7)$ & $\mathrm{p}<0,001$ \\
\hline
\end{tabular}

Der Vergleich zwischen den Behandlungsgruppen ergab keinen statistisch signifikanten Unterschied im neurologischen Outcome gemessen an der PCPC bei Krankenhausentlassung. Getestet auf den Parameter „Gutes neurologisches Outcome“ betrug der p-Wert 0,79 (Exakter Fisher-Test).

Tabelle 19: Neurologisches Outcome differenziert nach Behandlungsgruppen

\begin{tabular}{lllll}
\hline & Gruppe 1 & Gruppe 2 & Alle & \\
& $\mathrm{n}=58(\%)$ & $\mathrm{n}=24(\%)$ & $\mathrm{n}=82(\%)$ & $\mathrm{p}$-Wert \\
\hline PCPC 1 & $25(53,2)$ & $13(81,3)$ & $38(60,3)$ & \\
PCPC 2 & $4(8,5)$ & 0 & $4(6,3)$ & \\
PCPC 3 & $3(6,4)$ & $1(6,3)$ & $4(6,3)$ & \\
PCPC 4 & $12(25,5)$ & $2(12,5)$ & $14(22,2)$ & \\
PCPC 5 & $3(6,4)$ & 0 & $3(4,8)$ & \\
Delta-Score = 0 & $10(21,3)$ & $1(6,3)$ & $11(17,5)$ & \\
Gutes neurologisches Outcome & $39(83,0)$ & $15(93,8)$ & $54(85,7)$ & $\mathrm{p}=0,79$ \\
\hline
\end{tabular}




\subsection{Subgruppenanalysen}

\subsubsection{Atem-Kreislauf-Stillstand}

In der Studienpopulation der präklinisch intubierten Kinder wiesen 27 einen Atem-KreislaufStillstand auf, sodass eine kardiopulmonale Reanimation erfolgte $(1,3 \%$ aller Kindernotarzteinsätze). Die in der epidemiologischen Beschreibung von Reanimationsstudien üblicherweise verwendeten Daten sind in Tabelle 20 aufgeführt.

Abbildung 24: Häufigkeit von Atem-Kreislauf-Stillständen nach Altersgruppen

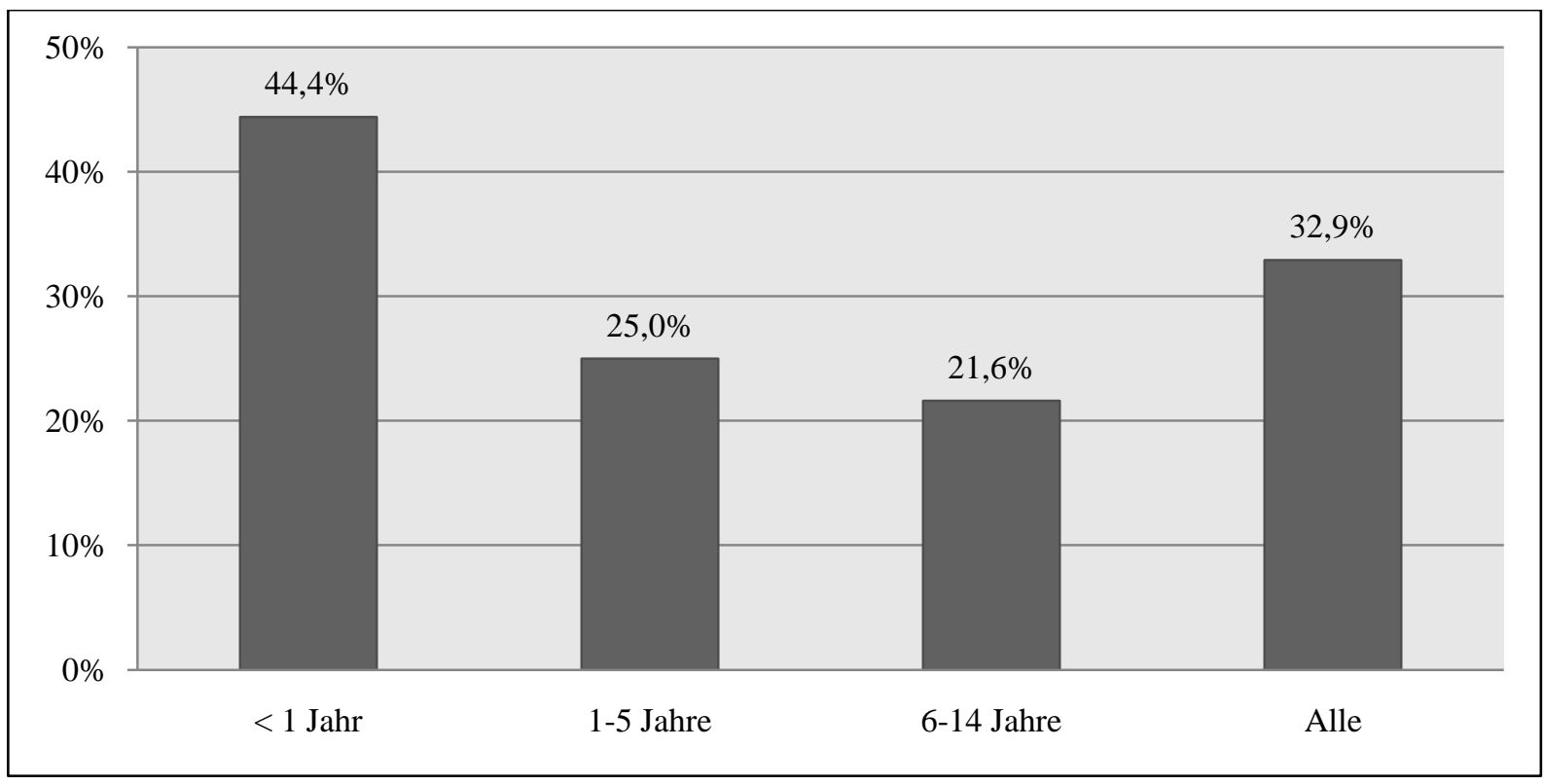

Tabelle 20: Charakteristika und Begleitumstände bei Atem-Kreislauf-Stillstand

\begin{tabular}{ll}
\hline Patientencharakteristika & $\mathrm{n}=27$ \\
\hline Alter & \\
Median (Mittelwert \pm SD) & $2(3,2 \pm 4,7)$ Jahre \\
Geschlecht & $\mathrm{n}(\%)$ \\
$\quad$ Männlich & $19(70,4)$ \\
\hline Begleitumstände & $\mathrm{n}(\%)$ \\
\hline Beobachteter Kreislaufstillstand & $10(37,0)$ \\
Laienreanimation begonnen & $11(40,7)$ \\
Initialer EKG-Rhythmus & \\
$\quad$ Asystolie & $15(55.6)$ \\
Bradykardie/PEA & $10(37.0)$ \\
$\quad$ Unbekannt & $2(7.4)$ \\
\hline Präklinische Versorgungszeiten & Median (Bereich) \\
\hline Eintreffzeit & $10,5(4-24)$ min \\
Alarmierung bis Ankunft Klinik & $53,5(30-83)$ min \\
\hline
\end{tabular}




\subsubsection{1.}

Die gehäufte Inzidenz der Reanimationen im Säuglingsalter wurde durch eine hohe Rate an plötzlichem Kindstod (SIDS) verursacht (37,0\%; 83,3\% bei den Säuglingen). Klein- und Schulkinder nach Ertrinkungsunfällen hatten einen Anteil von 25,9\% der kardiopulmonalen Reanimationen. Nach diesen war Trauma mit insgesamt vier Fällen (14,8\%) Ursache für einen Herz-Kreislaufstillstand mit den höchsten Prävalenzen in den Altersgruppen der Kleinund Schulkinder. Atem-Kreislauf-Stillstände infolge von respiratorischer Insuffizienz (2 Fälle) sowie Fremdkörperaspiration, Anaphylaxie und Sepsis (je 1 Fall) waren seltenere Ätiologien.

Abbildung 25: Ätiologie von pädiatrischen Atem-Kreislauf-Stillständen

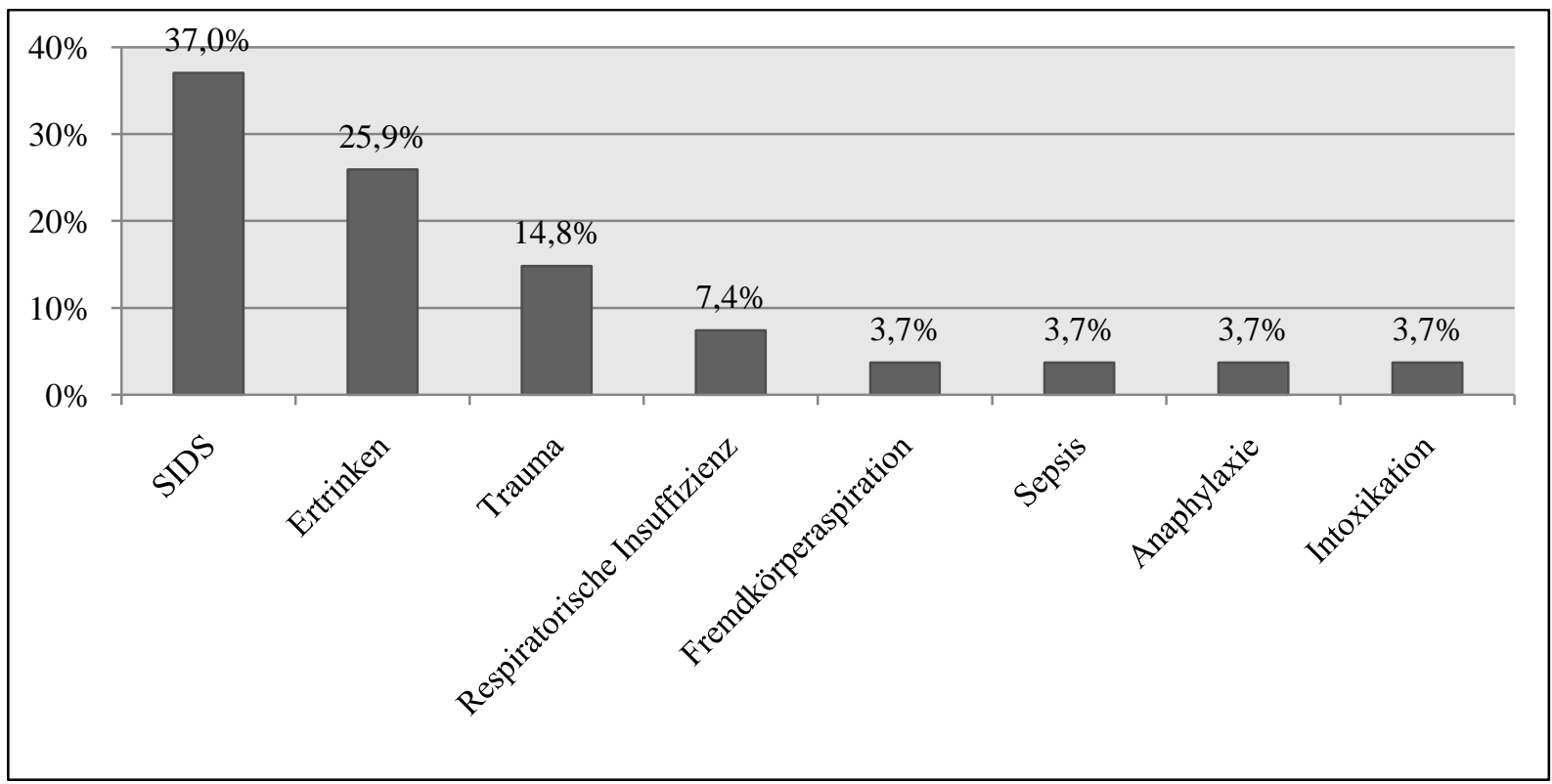

Tabelle 21: Ätiologie von Atem-Kreislauf-Stillständen nach Altersgruppen

\begin{tabular}{|c|c|c|c|c|}
\hline Ätiologie & $\begin{array}{l}<1 \mathrm{Jahr} \\
\mathrm{n}=12(\%)\end{array}$ & $\begin{array}{l}\text { 1-5 Jahre } \\
\mathrm{n}=7(\%)\end{array}$ & $\begin{array}{l}\text { 6-14 Jahre } \\
\mathrm{n}=8(\%)\end{array}$ & $\begin{array}{l}\text { Alle } \\
\mathrm{n}=27(\%)\end{array}$ \\
\hline SIDS & $10(83,3)$ & 0 & 0 & $10(37,0)$ \\
\hline Ertrinken & 0 & $4(57,1)$ & $3(37,5)$ & $7(25,9)$ \\
\hline Trauma & $1(8,3)$ & $1(14,3)$ & $2(25,0)$ & $4(14,8)$ \\
\hline Respiratorische Insuffizienz & $1(8,3)$ & 0 & $1(12,5)$ & $2(7,4)$ \\
\hline Fremdkörperaspiration & 0 & $1(14,3)$ & 0 & $1(3,7)$ \\
\hline Sepsis & 0 & $1(14,3)$ & 0 & $1(3,7)$ \\
\hline Anaphylaxie & 0 & 0 & $1(12,5)$ & $1(3,7)$ \\
\hline Intoxikation & 0 & 0 & $1(12,5)$ & $1(3,7)$ \\
\hline
\end{tabular}




\subsubsection{Outcome}

22 von 27 Kindern mit Atem-Kreislauf-Stillstand wurden in ein Krankenhaus eingeliefert. Bei 14 Kindern konnte am Notfallort ein Spontankreislauf wiederhergestellt werden (Return of Spontaneous Circulation, ROSC). Acht Kinder wurden unter fortlaufender kardiopulmonaler Reanimation in die Klinik transportiert. Sechs Kinder wurden nach Übergabe in der Klinik für tot erklärt, darunter fünf Kinder, die einen plötzlichen Kindstod erlitten. Elf Kinder (40,7\%) überlebten bis zur Entlassung aus dem Krankenhaus, wovon sechs (22,2\%) ein gutes neurologisches Outcome aufwiesen. Mehr als die Hälfte von insgesamt 19 Kindern, die vor oder kurz nach Aufnahme im Krankenhaus verstarben, waren Säuglinge mit SIDS (52,6\%), gefolgt von trauma-bedingten Todesfällen $(31,6 \%)$ mit Schwerpunkt bei den Schulkindern.

Tabelle 22: Outcome der Kinder mit Atem-Kreislauf-Stillstand

\begin{tabular}{lllll}
\hline & $<1 \mathrm{Jahr}$ & 1-5 Jahre & $6-14$ Jahre & Alle \\
& $\mathrm{n}=12(\%)$ & $\mathrm{n}=7(\%)$ & $\mathrm{n}=8(\%)$ & $\mathrm{n}=27(\%)$ \\
\hline Tod am Notfallort & $4(33,3)$ & $1(14,3)$ & 0 & $5(18,5)$ \\
Fortlaufende CPR & $5(41,7)$ & $2(28,6)$ & $1(12,5)$ & $8(29,6)$ \\
Tod bei Aufnahme & $4(33,3)$ & $1(14,3)$ & $1(12,5)$ & $6(22,2)$ \\
ROSC & $3(25,0)$ & $4(57,1)$ & $7(87,5)$ & $14(51,9)$ \\
Überlebensrate bis Klinik-Aufnahme & $3(25,0)$ & $6(85,7)$ & $7(87,5)$ & $16(59,3)$ \\
Überlebensrate bis Klinik-Entlassung & $2(16,7)$ & $4(57,1)$ & $5(62,5)$ & $11(40,7)$ \\
Gutes neurologisches Outcome & 0 & $2(28,6)$ & $4(50,0)$ & $6(22,2)$ \\
\hline
\end{tabular}




\subsubsection{Traumata}

Traumata waren mit 50\% aller Fälle die führende Diagnose, die die Notärzte zur präklinischen Intubation veranlasste. Die Prävalenz in der Altersgruppe Schulkinder war mit 73\% mit Abstand am höchsten.

Abbildung 26: Häufigkeitsverteilung der Traumanotfälle nach Altersgruppen

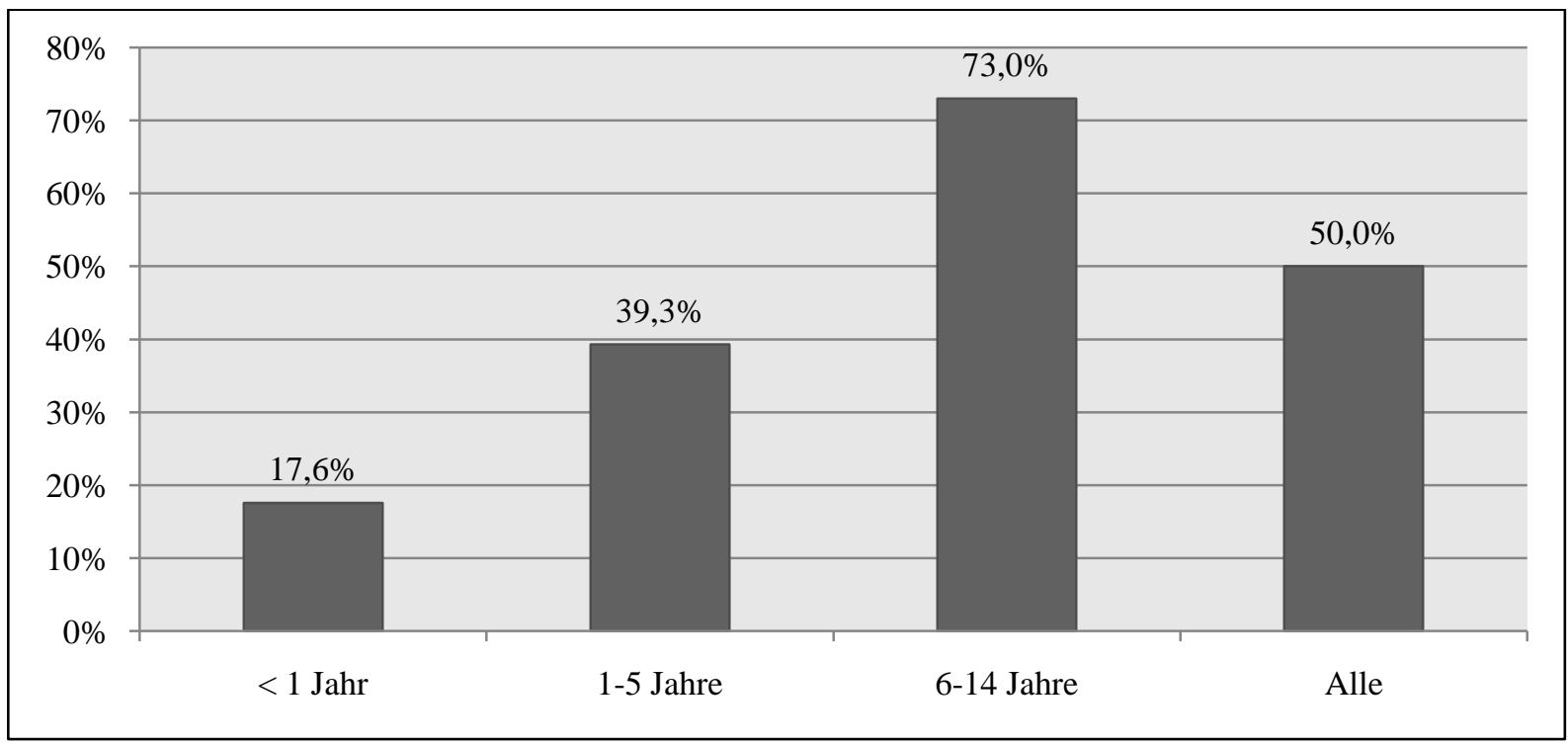

\subsubsection{1. Ätiologie, Verletzungsmuster und -schwere}

Mit 58,5\% waren Verkehrsunfälle die häufigste Ursache für das zugrundeliegende Trauma. Traumata infolge von Stürzen zeichneten sich mit 31,7\% ebenfalls als häufige Ursache ab.

\section{Abbildung 27: Traumaätiologie}

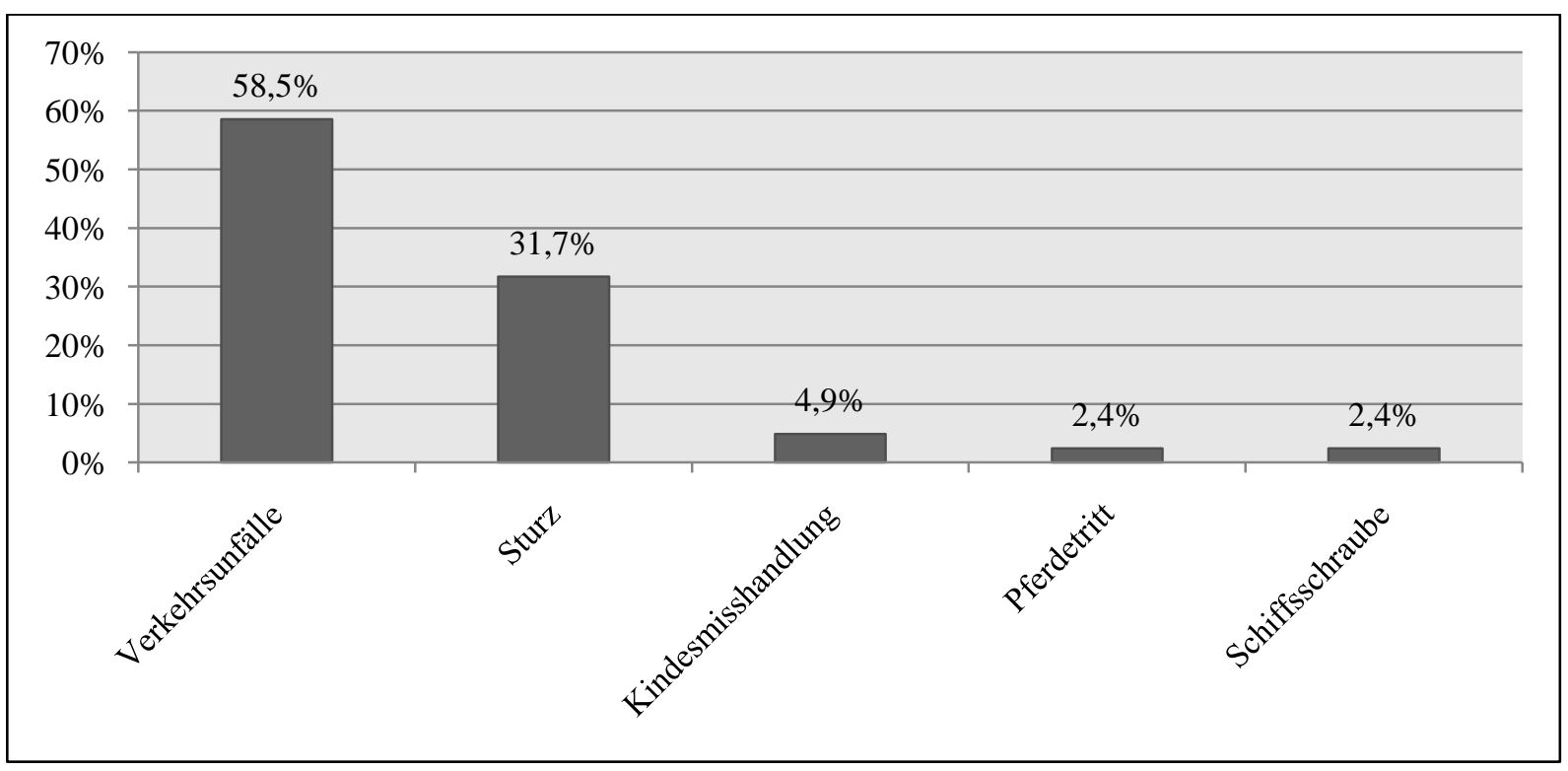


Die Ätiologie der Verletzungen in Abhängigkeit der Altersgruppen ist Tabelle $23 \mathrm{zu}$ entnehmen. Die dominierende Altersgruppe, die in Verkehrsunfälle verwickelt war, war mit $66,7 \%$ die der Schulkinder. Am häufigsten kam es zu Verkehrsunfällen mit Beteiligung von PKW im Straßenverkehr. Mehr als 60\% der Verkehrsunfälle sind auf die aktive Teilnahme der Kinder im Straßenverkehr als Fußgänger oder Radfahrer zurückzuführen.

Tabelle 23: Traumaätiologie nach Altersgruppen

\begin{tabular}{lllll}
\hline & $<$ 1 Jahr & 1-5 Jahre & 6-14 Jahre & Alle \\
Ätiologie & $\mathrm{n}=3(\%)$ & $\mathrm{n}=11(\%)$ & $\mathrm{n}=27(\%)$ & $\mathrm{n}=41(\%)$ \\
\hline Verkehrsunfälle & $1(33,3)$ & $5(45,5)$ & $18(66,7)$ & $24(58,5)$ \\
$\quad$ Person gegen PKW & 1 & 1 & 4 & 6 \\
$\quad$ PKW-Insasse & 0 & 1 & 5 & 6 \\
$\quad$ Fahrrad gegen PKW & 0 & 1 & 4 & 5 \\
$\quad$ Fahrrad & 0 & 1 & 3 & 4 \\
$\quad$ Trecker & 0 & 1 & 2 & 3 \\
Sturz & 0 & $6(54,5)$ & $7(25,9)$ & $13(31,7)$ \\
Kindesmisshandlung & $2(66,7)$ & 0 & 0 & $2(4,9)$ \\
Pferdetritt & 0 & 0 & 1 & $1(2,4)$ \\
Schiffsschraube & 0 & 0 & 1 & $1(2,4)$ \\
\hline
\end{tabular}

Abbildung 28 zeigt die Verteilung der Verletzungsmuster. 20 Kinder $(48,8 \%)$ erlitten ein isoliertes Schädel-Hirn-Trauma. 13 Kinder $(31,7 \%)$ wurden aufgrund eines Polytraumas intubiert, wovon bei 10 Kindern zusätzlich ein SHT bestand. Somit lag bei 73,2\% der Kinder ein SHT entweder in isolierter Form oder in Kombination mit anderen Verletzungen vor.

Abbildung 28: Verteilung der Verletzungsmuster

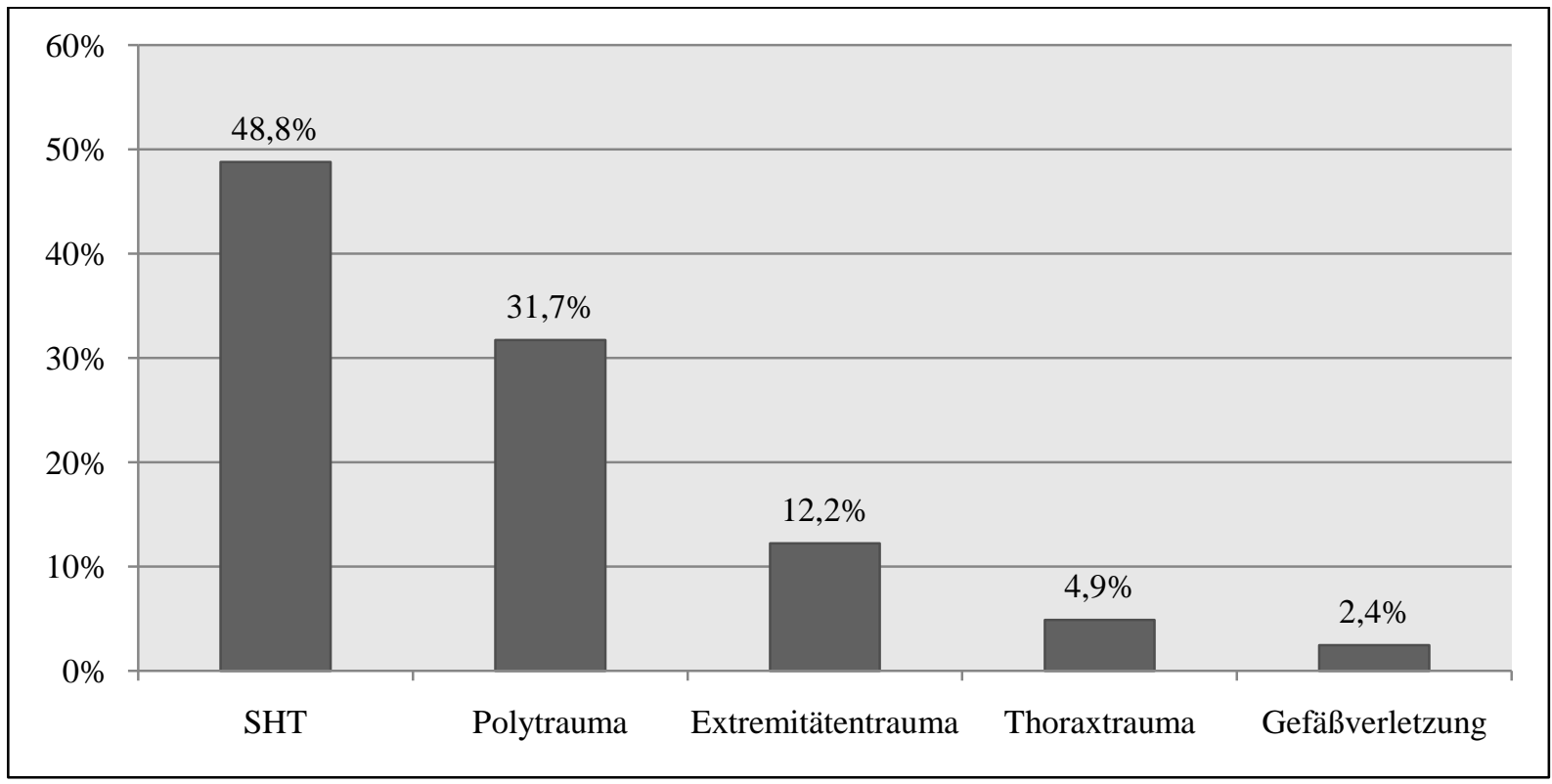


Tabelle 24: Verletzungsmuster nach Altersgruppen

\begin{tabular}{lllll}
\hline & $<1 \mathrm{Jahr}$ & 1-5 Jahre & 6-14 Jahre & Alle \\
Diagnosen & $\mathrm{n}=3(\%)$ & $\mathrm{n}=11(\%)$ & $\mathrm{n}=27(\%)$ & $\mathrm{n}=41(\%)$ \\
\hline SHT & $3(100,0)$ & $5(45,5)$ & $12(44,4)$ & $20(48,8)$ \\
Polytrauma & 0 & $5(45,5)$ & $8(29,6)$ & $13(31,7)$ \\
$\quad$ Polytrauma mit SHT & 0 & $4(36,4)$ & $6(22,2)$ & $10(24,4)$ \\
$\quad$ Polytrauma ohne SHT & 0 & $1(9,1)$ & $2(7,4)$ & $3(7,3)$ \\
Extremitätentrauma & 0 & 0 & $5(18,5)$ & $5(12,2)$ \\
Thoraxtrauma & 0 & 0 & $2(7,4)$ & $2(4,9)$ \\
Gefäßtrauma & 0 & $1(9,1)$ & 0 & $1(2,4)$ \\
\hline
\end{tabular}

Als Anhaltswert für die Verletzungsschwere ergab die Analyse der NACA-Werte einen Median von V. Die Glasgow-Coma-Scale lag im Median bei 8 Punkten. Von den 30 Kindern mit SHT wiesen 73,3 \% einen GCS-Wert unter 9 Punkten auf. Somit lag nach notärztlicher Ersteinschätzung bei der Mehrzahl der verletzten Kinder ein schweres Trauma mit potentieller vitaler Bedrohung vor.

Tabelle 25: NACA- und GCS-Scores der traumatisierten Kinder

\begin{tabular}{ll}
\hline & Median (Bereich) \\
\hline NACA-Score & V (III-VII) \\
GCS & $8(3-15)$ \\
\hline
\end{tabular}

Die Eintreffzeit der notarztbesetzten Rettungsmittel beim kindlichen Trauma betrug im Median 10 Minuten. 53 Minuten (Median) nach Alarmierung wurden die Kinder im Traumazentrum übergeben.

Tabelle 26: Präklinische Versorgungsintervalle beim kindlichen Trauma

\begin{tabular}{ll}
\hline Intervall & Median (Bereich) \\
\hline Alarmierung-Eintreffen Einsatzort & $10(4-29) \mathrm{min}$ \\
Alarmierung-Aufnahme Klinik & $53(31-99) \mathrm{min}$ \\
\hline
\end{tabular}




\subsubsection{Outcome}

Die Mortalität der intubierten pädiatrischen Traumapatienten betrug 14,7\%: Zwei Kinder erlagen am Unfallort ihren Verletzungen. Vier Kinder verstarben im weiteren Verlauf auf der Intensivstation, davon drei an den Folgen schwerster Schädel-Hirn-Verletzungen.

Die Überlebensrate bis zur Krankenhausentlassung betrug 85,4\%. Von den lebend aus dem Krankenhaus entlassenen Kindern wiesen $88,6 \%$ ein gutes neurologisches Outcome auf. In der Altersgruppe der Säuglinge zeigten jedoch alle drei Kinder ein ungünstiges neurologisches Outcome (zwei Säuglinge PCPC 4, ein Säugling PCPC 5).

Tabelle 27: Outcome pädiatrischer Traumapatienten

\begin{tabular}{lllll}
\hline & $<$ 1 Jahr & 1-5 Jahre & 6-14 Jahre & Alle \\
& $\mathrm{n}=3(\%)$ & $\mathrm{n}=11(\%)$ & $\mathrm{n}=27(\%)$ & $\mathrm{n}=41(\%)$ \\
\hline Tod am Notfallort/Aufnahme & 0 & $1(9,1)$ & $1(3,7)$ & $2(4,9)$ \\
Tod < 24 h nach Ereignis & 0 & 0 & 0 & 0 \\
Tod > 24 h nach Ereignis & 0 & 0 & $4(14,8)$ & $4(9,8)$ \\
Überlebensrate bis Klinik-Entlassung & $3(100,0)$ & $10(90,9)$ & $22(81,5)$ & $35(85,4)$ \\
Gutes neurologisches Outcome & 0 & $10(100,0)$ & $21(95,5)$ & $31(88,6)$ \\
\hline
\end{tabular}




\subsubsection{Ertrinkungsunfälle}

Insgesamt sieben Kinder (8,5\%) im Studienkollektiv waren Opfer von Ertrinkungsunfällen. Drei Kinder ertranken im Schwimmbad, jeweils zwei Kinder im Gartenteich oder Swimmingpool und zwei Kinder in Flüssen.

Abbildung 29: Kreisdiagramm Ort des Ertrinkens

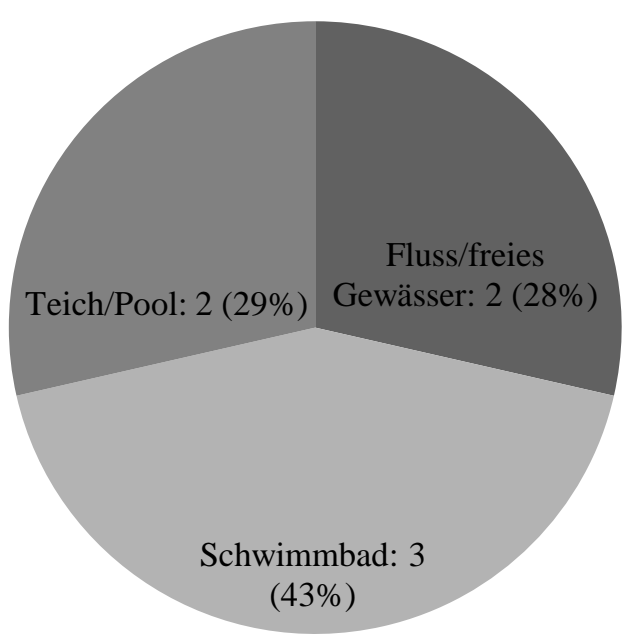

In allen Fällen resultierte ein Atem-Kreislauf-Stillstand. Darunter waren vier Kleinkinder und drei Schulkinder (fünf Jungen und zwei Mädchen).

Überlebensraten und Outcome sind in Tabelle 28 aufgeführt. Bei sechs Kindern konnte am Notfallort ein Spontankreislauf wiederhergestellt (ROSC) werden. Vier dieser Kinder erhielten Laienreanimation, bevor der Notarzt eintraf.

Ein Kind nach Ertrinkungsunfall in einem Fluss und Submersionszeit über 30 Minuten mit schwerer Hypothermie und Asystolie wurde unter Fortführung der CPR in die Klinik transportiert. Es verstarb trotz Wiedererwärmung mittels extracorporaler Zirkulation innerhalb von 72 Stunden nach Aufnahme auf die Intensivstation. Die sechs Kinder mit ROSC überlebten bis zur Krankenhausentlassung (Überlebensrate 85,7\%). Vier dieser wiesen ein gutes neurologisches Outcome auf und konnten nach Hause entlassen werden (alle PCPC 1), zwei Kinder überlebten mit schwerer hypoxischer Enzephalopathie (PCPC 4 und PCPC 5). 
Tabelle 28: Überlebensrate und Outcome von Kindern nach Ertrinkungsunfall

\begin{tabular}{llll}
\hline & 1-5 Jahre & 6-14 Jahre & Alle \\
Outcome & $\mathrm{n}=4(\%)$ & $\mathrm{n}=3(\%)$ & $\mathrm{n}=7(\%)$ \\
\hline ROSC & $3(75,0)$ & $3(100,0)$ & $7(100,0)$ \\
Überlebensrate bis Klinik-Aufnahme & $4(100,0)$ & $3(100,0)$ & $7(100,0)$ \\
Überlebensrate bis Klinik-Entlassung & $3(75,0)$ & $3(100,0)$ & $6(85,7)$ \\
Gutes neurologisches Outcome & $2(50,0)$ & $2(66,7)$ & $4(66,7)$ \\
\hline
\end{tabular}

Der Tabelle 29 sind die geschätzten Submersionszeiten und das Patientenoutcome zu entnehmen. Drei Kinder mit einer Submersionszeit von weniger als fünf Minuten überlebten allesamt ohne neurologisches Defizit (alle drei erhielten Laienreanimation). Zwei Kinder befanden sich zwischen fünf und zehn Minuten unter Wasser und überlebten bis zur Entlassung aus dem Krankenhaus (eins mit schwerer hypoxischer Enzephalopathie, PCPC 5). Ein Kind mit unbekannter Submersionszeit nach Ertrinken im Freibad (Laienreanimation wurde durchgeführt) überlebte mit schwerer Behinderung (PCPC 4).

Tabelle 29: Submersionszeiten und Outcome

\begin{tabular}{lccc}
\hline Submersionszeit & $\begin{array}{c}\text { Überleben bis Klinik- } \\
\text { Entlassung } \mathrm{n}=6\end{array}$ & $\begin{array}{c}\text { Gutes neurologisches } \\
\text { Outcome } \mathrm{n}=4\end{array}$ & $\begin{array}{c}\text { Alle } \\
\mathrm{n}=7\end{array}$ \\
\hline$<5 \mathrm{Min}$ & 3 & 3 & 3 \\
$5-10 \mathrm{Min}$ & 2 & 1 & 2 \\
$>10 \mathrm{Min}$ & 0 & 0 & 1 \\
Unbekannt & 1 & 0 & 1 \\
\hline
\end{tabular}

\subsection{Wahrscheinlichkeit einer präklinischen Kinderintubation}

Innerhalb des achtjährigen Studienzeitraumes wurden insgesamt 36677 Notfalleinsätze (Erwachsene und Kinder) von insgesamt 132 Studienärzten durchgeführt. Dies ergibt einen Median von 218 Einsätzen pro Notarzt (Bereich 1-1859). Bei 58 Kindern, die durch die Studienärzte präklinisch intubiert wurden, wird ein Göttinger Notarzt im Mittel alle drei Jahre mit einer präklinischen Intubation bei einem Schulkind konfrontiert. Diese Wahrscheinlichkeit verringert sich auf alle acht Jahre bei einem Kleinkind und alle 13 Jahre bei einem Säugling. 


\section{Diskussion}

\subsection{Charakteristika der präklinischen Intubation}

Die präklinische Intubation von Kindern ist eine selten erforderliche Maßnahme. In der vorliegenden Untersuchung wurde in $4,0 \%$ der Notarzteinsätze bei Kindern eine endotracheale Intubation durchgeführt. Dies entspricht den Zahlen vergleichbarer Studien. STENKE (2004) gibt die Häufigkeit der ETI im Münchner Kindernotarztdienst mit 2,0\% der Einsätze an. BABL et al. (2001) beziffern diese in einem US-amerikanischen ParamedicSystem auf 2,7\%. Wie aus unseren Daten hervorgeht, würde der einzelne Notarzt alle drei Jahre mit einer Kinderintubation konfrontiert. In der Untersuchung von GAUSCHE et al. (2000) intubierten Paramedics durchschnittlich sogar nur alle 15 Jahre ein Kind.

In Patientenkollektiven Erwachsener wird die präklinische endotracheale Intubation häufiger angegeben als bei pädiatrischen Notfällen (BOSWELL et al. (1995), RICHARD et al. (2006)). TIMMERMANN et al. (2006) berichten eine Intubationshäufigkeit von $6,8 \%$ bei erwachsenen Notfallpatienten im Rettungsdienstbereich Göttingen. Dieses könnte zum einen begründet liegen in den tendenziell niedrigeren Schweregraden pädiatrischer Notfalleinsätze und zum anderen in einer gewissen Zurückhaltung der Notärzte, ein Kind unter Notfallbedingungen zu intubieren (DIRUSSO et al. (2005)).

Die Frequenz der durchgeführten Intubationen im Luftrettungsdienst ist deutlich höher als in bodengebundenen Rettungssystemen (EICH et al. (2009b)). Dies ist auf einen größeren Anteil schwerwiegenderer Kindernotfälle (NACA IV-VII) und auf einen höheren Anteil an Traumapatienten zurückzuführen (HELM et al. (1999), ALBRECH et al. (2000), SCHLECHTRIEMEN et al. (2006)).

Die führenden Diagnosegruppen waren Traumata (50\%), Krampfanfälle (13,4\%) und SIDS $(12,2 \%)$. Diese zeigen generell eine hohe Prävalenz in der pädiatrischen Notfallmedizin (BROWNSTEIN et al. (1996), RICHARD et al. (2006), EICH et al. (2009b)). Obwohl die beobachteten Häufigkeitsverteilungen im Wesentlichen konsistent mit anderen Studien sind, variieren diese jedoch abhängig von den gewählten oberen Altersgrenzen, die zwischen 12 und 18 Jahren liegen (EASLEY et al. (2000), GAUSCHE et al. (2000)). Des Weiteren fokussieren einige Untersuchungen über präklinische Intubation von Kindern auf spezielle Subgruppen, wie z.B. traumatisierte Kinder, und weisen demzufolge einen höheren 
Altersdurchschnitt auf. Beide Aspekte beeinflussen die Charakteristika trachealer Intubationen von Kindern und das Patientenoutcome.

Die Erfolgsraten der präklinischen Intubation von Kindern schwanken in den verfügbaren Referenzen jeweils in Abhängigkeit vom Durchführenden. In Paramedic-basierten Rettungssystemen werden variable Erfolgsraten zwischen 56\% und 95\% angegeben (POINTER (1989), BOSWELL et al. (1995), GAUSCHE et al. (2000), HARRISON et al. (2004)). Notärztlich durchgeführte Intubationen demonstrieren in der Literatur konstant hohe Erfolgsquoten (DUPANOVIC et al. (2010), MARTINON et al. (2010)). In einer französischen Studie von MEYER et al. (2000) betrug die Erfolgsrate 98\%, wenn Notärzte verschiedener Fachdisziplinen Kinder intubierten.

In der vorliegenden Untersuchung konnten alle Kinder bis auf einen Säugling erfolgreich endotracheal intubiert werden. Dies entspricht einer Gesamt-Erfolgsrate von 98,8\%. Ein Säugling konnte nach gescheiterter ETI problemlos mit einer Larynxmaske oxygeniert und ventiliert werden.

Ein direkter Vergleich der Studien zum präklinischen Atemwegsmanagement bei Kindern ist aufgrund der Heterogenität von Studiendesign und -kollektiven schwierig. In vielen USamerikanischen Untersuchungen wurden nur pulslose Kinder, Kinder mit Atemstillstand oder tief komatöse Patienten eingeschlossen. Die Applikation von Narkotika oder Muskelrelaxantien war den Paramedics in der Regel nicht gestattet. Diese Faktoren könnten die hohen Fehlerraten zumindest partiell erklären. Des Weiteren ist unklar, inwiefern Ergebnisse auf unterschiedlich strukturierte Rettungssysteme übertragbar und inwieweit direkte Vergleiche zwischen Paramedic-basierten und Notarzt-basierten Rettungssystemen möglich sind, wo ggf. gut geschulte Anästhesisten mit entsprechender Intubationserfahrung zum Einsatz kommen (TIMMERMANN et al. (2008)).

Die ETI von Kindern insbesondere von Säuglingen und Kleinkindern wird, wie einführend beschrieben und durch die eigenen Ergebnisse unterstuitzt, generell als schwieriger eingeschätzt. Unter präklinischen Notfallbedingungen kommen zusätzlich erschwerende Umstände hinzu. Ein Ziel dieser Untersuchung war die prospektive Erfassung der Sichtbedingungen bei der direkten Laryngoskopie entsprechend der Cormack-LehaneGraduierung und der Inzidenz der „schwierigen Intubation“ bei Kindern sowie deren zugrunde liegenden Ursachen. Eingeschränkte laryngoskopische Sichtverhältnisse (CL $\geq 3$ ) lagen in $15,5 \%$ und eine subjektive schwierige Intubation in 13,8\% der Fälle vor. Diese 
Häufigkeiten sind damit etwas niedriger als in vergleichbaren Studien bei Erwachsenen (MACKAY et al. (2001), TIMMERMANN et al. (2006)). Jedoch zeigte sich in der Altersgruppe der Säuglinge eine deutliche, wenn auch nicht statistisch signifikante, höhere Inzidenz beider Kriterien (jeweils 23,1\%). Diese Beobachtung könnte auf anatomische Schwierigkeiten im Säuglingsalter, besondere Begleitumstände - die meisten Säuglinge erlitten SIDS - und einer relativ geringen klinischen Expertise in der Intubation von Säuglingen und Kleinkindern zurückzuführen sein (EASLEY et al. (2000), HARRISON et al. (2004)).

\subsection{Intubationszwischenfälle}

Fehler und Komplikationen bei der ETI sind assoziiert mit sekundären kardiopulmonalen und möglicherweise gar fatalen Komplikationen wie schweres neurologisches Defizit oder Tod (EHRLICH et al. (2004), MORT (2004)).

Neben der Tubusfehllage und Tubusdislokation (1) zählen multiple Intubationsversuche (2) sowie die misslungene Intubation (3) zu den drei Schlüsselfehlern der Intubation, die potenziell zu einer Erhöhung der Morbidität und Mortalität führen (WANG et al. (2009a)). Laryngoskopie und Intubation können infolge eines Atemwegstraumas zu Schleimhautödemen, Schwellungen und Blutungen führen (MORT (2007a)). Auch kann die Laryngoskopie Regurgitation und Aspiration provozieren. Zu den Intubationsfehlern zählen ferner jene Komplikationen, die zu vermeiden sind, jedoch das unmittelbare Risiko des Patienten nicht erhöhen, wie z.B. falsche Tubusgröße oder inadäquat geblockte bzw. fixierte Tuben. Jedoch können auch diese vermeintlich weniger gravierenden Unregelmäßigkeiten durchaus nachhaltige Folgeschäden verursachen (EASLEY et al. (2000), HOLZKI et al. (2009)). Darüber hinaus kann die endotracheale Intubation durch einen starken vegetativen Reiz zu akuten hämodynamischen Alterationen führen. Dabei kann es sowohl zu einer hyperdynamen Reizantwort mit Tachykardie und Hypertonie kommen als auch zu schweren Bradykardien bis hin zur Asystolie (MORT (2007b)).

In den letzten Jahren häufen sich die Publikationen, die über eine hohe Zahl von präklinischen Intubationszwischenfällen berichten. Vor allem Untersuchungen bei erwachsenen Patienten zeigen alarmierende Zahlen auf. WANG et al. (2009a) detektierten eine Inzidenz an Intubationsfehlern von 22,7\%, wenn Paramedics intubiert hatten. In einer prospektiven Studie von TIMMERMANN et al. (2007c) betrug die Rate der Intubationszwischenfälle in einem adulten Patientenkollektiv 17,4\%, wenn Notärzte die Intubation durchführten. Bei GAUSCHE 
et al. (2000) traten ösophageale Intubationen in $2 \%$, Tubusdislokationen in $14 \%$ und Intubationen des rechten Hauptbronchus in $18 \%$ der Fälle auf. Die Rate der misslungenen Intubationen betrug über $30 \%$.

In der vorliegenden Untersuchung traten die drei Schlüsselfehler der Intubation in 12,2\% aller Fälle auf. Es zeigte sich jedoch, dass Fehler bei den anästhesiologisch weitergebildeten Studienärzten der Gruppe 1 mit 6,9\% seltener waren im Vergleich zur Gruppe 2 (25\%) (p = $0,06)$.

Eine der fatalsten Komplikationen ist die unerkannte ösophageale Intubation. Diese ist beim apnoeischen Kind mit hoher Wahrscheinlichkeit mit einem tödlichen Ausgang verbunden (NOLAN und SOAR (2008)). In der Studie von GAUSCHE et al. (2000) waren 15 von 177 Kindern $(\sim 8 \%)$ entweder unerkannt ösophageal intubiert worden, oder es kam zu einer unerkannten Tubusdislokation mit der Folge, dass 14 dieser Kinder starben. In der Untersuchung von TIMMERMANN et al. (2007c) an einem adultem Patientenkollektiv im Versorgungsbereich des Göttinger Luftrettungszentrums wurde von den nachgeforderten Notärzten des RTH eine ösophageale Fehlintubationsrate von 6,7\% (10 von 149 Patienten) detektiert, wenn ein erstversorgender Notarzt die Intubation bereits durchgeführt hatte. Sieben von zehn fehlintubierten Patienten verstarben innerhalb der ersten 24 Stunden der Behandlung. Ähnliche alarmierende Ergebnisse zeigt ein Editorial von VON GOEDECKE et al. (2007) aus Innsbruck mit Bezug auf die o.g. Studie, welches über weitere fünf Fälle von Atemwegsmanagement-Desastern von Notärzten berichtet. KATZ und FALK (2001) in Orlando, Florida/USA legten eine Rate von 16\% ösophagealen Intubationen bei erwachsenen Patienten dar, die durch Paramedics intubiert worden waren. Zehn der 18 fehlintubierten Patienten verstarben noch in der Notaufnahme.

In der vorliegenden Studie traten unerkannt endobronchiale Intubationen in zwei Fällen und ösophageale Intubationen in keinem Fall auf.

Der konsequente Einsatz der Kapnographie, wie in der vorliegenden Studie erfolgt, ist ein effektives Hilfsmittel zur Verifikation der Tubuslage aber auch zur Detektion einer versehentlichen Tubusdislokation während des Transports (SILVESTRI et al. (2005)). Obwohl alle Rettungsmittel standardmäßig mit dem Equipment für eine endotracheale Intubation ausgestattet sind, mangelt es oftmals an Hilfsmitteln zur Überprüfung der Tubuslage. In einer Umfrage von SCHMID et al. (2006) in Bayern waren nur knapp 32\% der 
notarztbesetzten bodengebundenen Rettungsmittel mit endtidalen $\mathrm{CO}_{2}$-Detektoren ausgestattet.

Die Laryngoskopie und die endotracheale Intubation sind invasive Maßnahmen. Multiple und unsachgemäß durchgeführte Intubationsversuche sind assoziiert mit Komplikationen wie Verletzungen der Luftwege, Bradykardie, Hypoxämie und Herzstillstand und können zur Erhöhung der Morbidität und Mortalität in erheblicher Weise beitragen (MORT (2004), WANG und YEALY (2006b), HOLZKI et al. (2009)). EHRLICH et al. (2004) konnten an pädiatrischen Traumapatienten eine 2,5-fache Erhöhung des relativen Risikos für Atemwegskomplikationen nachweisen, wenn mehr als ein Intubationsversuch unternommen wurde. WANG und YEALY (2006b) bezifferten die Anzahl der Patienten, die mehr als einen Intubationsversuch durch einen Paramedic erhielten, auf mehr als $30 \%$ und empfahlen präklinische Intubationsversuche generell auf drei zu reduzieren. Auch POINTER (1989) beschrieb in seiner Untersuchung präklinischer Kinderintubationen, die durch Paramedics durchgeführt wurden, dass 50\% der Patienten mehr als einen Intubationsversuch erhielten.

In der vorliegenden Studie benötigten 15,5\% der Kinder mehr als einen Intubationsversuch. Atemwegs-bezogene Komplikationen wurden nicht beschrieben. Säuglinge hatten signifikant geringere Erfolgsraten im ersten Intubationsversuch, was konsistent mit den Beobachtungen von HARRISON et al. (2004) ist.

Wie aus unseren Daten und der beschriebenen Literatur deutlich wird, treten Zwischenfälle und Komplikationen bei der ETI selbst bei gut geschulten Notärzten auf. Die tatsächliche Rate an Intubationszwischenfällen dürfte allerdings noch höher liegen als in den Studien berichtet. Zahlreiche Hinweise sprechen dafür, dass die Zahl der Intubationsfehler bei selbstberichteten Ereignissen unterschätzt wird (SEXTON et al. (2000), DOERGES et al. (2003a), WANG und YEALY (2006a)). Auch in der vorliegenden Studie könnten die Intubationszwischenfälle einem systematischen „reporting bias“ unterliegen im Sinne einer Unterschätzung der eigenen und einer Überschätzung der Fehler der Notärzte in der Gruppe 2.

CUMMINS und HAZINSKI (2000, S. 447) kamen zu der Schlussfolgerung: "We must deal with the growing awareness of the fact that tracheal intubation is not only a potentially lethal intervention but now is also a confirmed lethal intervention, and at a much higher death rate than has ever been suspected." 


\subsection{Einfluss der präklinischen Intubation auf das Outcome}

Verschiedene Autoren haben versucht die präklinische ETI von Kindern und das resultierende Outcome zu korrelieren. DIRUSSO et al. (2005) konnten in ihrer Analyse eines pädiatrischen Traumaregisters bezüglich der Mortalitätsraten feststellen, dass sich, verglichen mit den nichtintubierten Patienten, die Odds-ratios (OR) für die Intubation am Notfallort (OR 14,4) und Intubation im Krankenhaus (OR Nicht-Trauma-Zentrum 5,8; OR Trauma-Zentrum 4,8) signifikant unterschieden. Die Intubation am Notfallort stellte sich als starker unabhängiger negativer Prädiktor für „Überleben“ und „gutes neurologisches Outcome“ bei traumatisierten Kindern dar. Die Autoren konnten anhand ihrer Daten jedoch keine Begründung bzw. keine Kausalitätsrichtung dieser Aussage liefern. In einer prospektiven, randomisiert-kontrollierten Studie - mit 830 Kindern die größte bisher publizierte präklinische Untersuchung an Kindern untersuchten GAUSCHE et al. (2000) den Einfluss der prähospitalen ETI auf Überleben und neurologisches Outcome bei Kindern unter zwölf Jahren im urbanen Einzugsgebiet von Los Angeles County/USA. Eingeschlossen wurden Kinder mit Atem- und/oder Kreislaufstillstand und tief komatöse (d.h. aufgehobene Reaktion auf Schmerzreize) Kinder mit Schädel-HirnTauma oder respiratorischer Insuffizienz. Im Studienzeitraum wurde eine Randomisierung vorgenommen, bei der an ungeraden Tagen eine Beutel-Masken-Beatmung und an geraden Tagen eine endotracheale Intubation durchgeführt werden sollte. Narkotika oder Muskelrelaxantien zur Erleichterung der Intubation wurden nicht appliziert. Verglichen mit der Gruppe der Kinder, die nur mit Beutel-Masken-Beatmung ventiliert wurden, konnte kein Überlebensvorteil in einer der beiden Gruppen (BMV vs. ETI) festgestellt werden (26\% vs. 30\%; OR 0,82, 95\%-KI 0,61-1,11). Auch hinsichtlich des sekundären Endpunktes „gutes neurologisches Outcome“ gab es keinen signifikanten Unterschied (23\% vs. 20\%; OR 0,87, 95\%-KI 0,62-1,22). Diese Studie konnte zeigen, dass die präklinische Intubation im Vergleich zur BMV zu keiner Verbesserung von Überlebensrate und neurologischem Outcome führte und damit die BMV der präklinischen Intubation ebenbürtig war. Allerdings weist die GAUSCHE et al. (2000)-Studie einige wichtige Limitierungen auf. Die Schlussfolgerungen, die aus dieser Landmarkenstudie gezogen werden können, müssen differenzierter interpretiert werden, als dies landläufig getan wird (GLAESER (2000), EICH et al. (2010)). Die Paramedics ( $\mathrm{n}>$ 3000) wurden lediglich im Rahmen einer einmaligen sechsstündigen Veranstaltung am Phantom im pädiatrischen Airwaymanagement geschult. Das Studiensetting war ein urbanes Einzugsgebiet mit relativ kurzen Zeiten bis zur Versorgung in der Klinik (Median 20 Minuten). In der Intubationsgruppe betrug die Gesamterfolgsquote nur 57\% und nur $42 \%$ in der randomisierten ETI-Gruppe wurden tatsächlich erfolgreich intubiert. 
Endtidales $\mathrm{CO}_{2}$-Monitoring zur Überprüfung der Tubuslage erfolgte nur in $77 \%$ und wurde während des Transportes nicht kontinuierlich durchgeführt. Das Outcome ,primäres Überleben“ und „neurologisches Outcome“ war in beiden Gruppen äußerst ungünstig.

In unserer Untersuchung fanden sich deutlich bessere Raten für Intubationserfolg (98,8\%), primäres Überleben $(76,8 \%)$ und gutes neurologisches Outcome $(85,7 \%)$.

Wie jedoch in Kapitel 4.1. bereits diskutiert, variieren die Kriterien für die ETI in den unterschiedlichen Rettungssystemen, was einen erheblichen Einfluss auf die Outcome-Daten haben dürfte und somit eine Übertragbarkeit limitiert. Die beträchtlich besseren OutcomeErgebnisse in unserer Studie könnten der frühen und versierten endotrachealen Intubation durch Anästhesisten, einem intensiven Patientenmonitoring mit konsequentem Einsatz der Kapnographie, sowie schnellen Eintreff- und Transportzeiten (Rettungshubschrauber) in ein pädiatrisches Zentrum der Maximalversorgung zugeschrieben werden (Median des präklinischen Versorgungsintervalls 52 Minuten).

\subsection{Diskussion der Subgruppen}

\subsubsection{Atem-Kreislauf-Stillstand}

In der vorliegenden Untersuchung wurden 27 von 82 Kindern (33,0\%) kardiopulmonal reanimiert. Die Häufigkeit der kardiopulmonalen Reanimation (CPR) war in der Altersgruppe der Säuglinge mit 44,4\% am höchsten und verursacht durch einen hohen Anteil an plötzlichem Kindstod. Den zweithöchsten Anteil kardiopulmonaler Reanimationen wiesen Kleinkinder mit Ertrinkungsunfällen auf, gefolgt von traumatisch bedingten HerzKreislaufstillständen im Schulkindalter. Diese ätiologische Häufigkeitsverteilung entspricht früheren Untersuchungen außerklinischer Atem-Kreislauf-Stillstände im Kindesalter (KUISMA et al. (1995), SUOMINEN et al. (1997)).

Die Prognose pädiatrischer Atem-Kreislauf-Stillstände wird als äußerst ungünstig beschrieben. Nur zwei bis zwölf Prozent aller Kinder, die einen Arrest erleiden, überleben (HERLITZ et al. (2005)). Die meisten Kinder überleben mit schweren neurologischen Residuen (SIRBAUGH et al. (1999), YOUNG et al. (2004)). Jedoch berichten neuere Daten über innerklinische Atem-Kreislauf-Stillstände Überlebensraten von 25-33\% und mit überwiegend gutem neurologischem Outcome (SUOMINEN et al. (2000b), NADKARNI et al. (2006)). Insgesamt gibt es Hinweise darauf, dass sich die Überlebensraten pädiatrischer 
Atem-Kreislauf-Stillstände in den letzten Jahren tendenziell verbessert haben (BERG et al. (2008), TOPJIAN et al. (2008)).

Unsere Ergebnisse zeigen noch höhere Überlebensraten: Elf von 27 Kindern (40,7\%) mit außerklinischem Atem-Kreislauf-Stillstand überlebten bis zur Entlassung aus dem Krankenhaus und sechs Kinder $(22,2 \%)$ wiesen ein gutes neurologisches Outcome auf. Eine wesentliche Begründung für die sehr hohen Überlebensraten dürfte bei einer insgesamt geringen Fallzahl der mit 25\% relativ hohe Anteil an Ertrinkungsunfällen sein, welche eine relativ günstige Prognose haben.

Bezüglich eines möglichen Einflusses der endotrachealen Intubation auf das Outcome bei Kindern mit Atem-Kreislauf-Stillstand gibt es nur wenige Daten. In einer prospektiven populations-basierten Untersuchung von SIRBAUGH et al. (1999) an 300 Kindern mit außerklinischem Atem-Kreislauf-Stillstand war die endotracheale Intubation die einzige Maßnahme, die positiv mit einer Wiederherstellung eines Spontankreislaufs korrelierte. Andererseits konnte in der beschriebenen Studie von GAUSCHE et al. (2000) auch in der Subgruppe Atem-Kreislauf-Stillstand kein Überlebensvorteil der intubierten Kinder gefunden werden (OR 0,96, 95\%-KI 0,53-1,73).

Die endotracheale Intubation bietet den Vorteil eine Herzdruckmassage ohne Unterbrechung für die Beatmung durchführen zu können und somit höhere und konstantere Perfusionsdrücke zu erreichen. KRAMER-JOHANSEN et al. (2006) konnten zeigen, dass die Qualität der Thoraxkompressionen nach endotrachealer Intubation gesteigert werden kann. Andererseits gibt es auch Hinweise dafür, dass durch prolongierte oder multiple Intubationsversuche die CPR häufig unterbrochen wird. Dadurch kann die sogenannte „,no-flow-time“ verlängert und die Organperfusion verschlechtert werden (WANG et al. (2009b)).

Die Frage, ob die endotracheale Intubation einen Einfluss auf die vergleichsweise hohe Überlebensrate in der vorliegenden Untersuchung hat, lässt sich durch unsere Daten nicht beantworten. Auch in der Literatur mangelt es an robusten wissenschaftlichen Daten, die die Annahme unterstützten, dass die endotracheale Intubation einen Überlebensvorteil für Patienten im Atem-Kreislauf-Stillstand mit sich bringt. 


\subsubsection{Traumata}

Unfälle belegen den ersten Rang in der Todesursachenstatistik bei Kindern im Alter zwischen ein und 14 Jahren (STATISTISCHES BUNDESAMT WIESBADEN (2000)).

Das Atemwegsmanagement mit dem Ziel einer adäquaten Oxygenierung und Ventilation hat eine hohe Priorität in der notfallmedizinischen Traumaversorgung (DUPANOVIC et al. (2010)). HUSSAIN und REDMOND (1994) folgerten aus ihrer Untersuchung präklinischer adulter Todesfälle aufgrund von Traumata, dass $85 \%$ der Patienten, die mit überlebbaren Verletzungen vor Erreichen des Krankenhauses verstarben, eine Obstruktion der Atemwege aufwiesen. Eine potentiell reversible Atemwegsverlegung könnte somit auch bei Kindern in vielen Fällen zum Tod durch schweres Trauma beitragen.

Schwer- oder polytraumatisierte Kinder werden nach anerkannten Kriterien der präklinischen Notfallversorgung bzw. gemäß den Empfehlungen internationaler Leitlinien häufig bereits präklinisch intubiert. Die Hälfte aller Intubationen in dieser Studie waren aufgrund von Traumata indiziert. Der Großteil der Kinder wies - gemessen an den NACA-Indices und den GCS-Werten - schwere Verletzungen auf. Die Überlebensrate betrug 85,4\%.

SCHMIDT et al. (2002) geben eine nahezu identische Überlebensrate von $88 \%$ bei pädiatrischen Traumapatienten $(n=104)$ im Einzugsgebiet der Medizinischen Hochschule Hannover an. SUOMINEN et al. (2000a) untersuchten den Effekt der Intubation auf die Überlebensrate Schädel-Hirn-traumatisierter Kinder $(n=59)$ in Finnland und fanden eine signifikant geringere Mortalität bei schwerverletzten Kindern, die bereits am Unfallort von Notärzten intubiert worden waren $(n=24)$, verglichen mit ETI nach Klinikaufnahme $(n=35)$.

In retrospektiven Analysen US-amerikanischer Traumaregister von COOPER A et al. (2001) sowie von STOCKINGER und MCSWAIN (2004) bei Kindern mit Schädel-Hirn-Trauma wurden hingegen keine Unterschiede in Mortalität oder funktionellem Outcome im Vergleich der Gruppen „Präklinische Intubation“ vs. „BMV“ festgestellt. Beide Arbeitsgruppen fanden zudem eine höhere Komplikationsrate in der Gruppe der intubierten Kinder.

Die Publikationen, die den Einfluss der präklinischen Intubation auf die Mortalität von pädiatrischen Traumapatienten untersuchen, kommen somit zu kontroversen Ergebnissen. Diese Feststellung wird unterstützt durch einen aktuellen Review-Artikel von VON ELM et al. (2009), der insgesamt 17 Studien mit über 15000 Patienten mit Schädel-Hirn-Trauma inkludierte (darunter acht pädiatrische Studien). Die Odds-ratios für einen Effekt der 
präklinischen Intubation auf die innerklinische Mortalität reichten von 0,17 (Überlegenheit der Kontrollinterventionen) bis 2,43 (Überlegenheit der ETI). Die Autoren kamen zu der Schlussfolgerung, dass keine konsistenten positiven oder negativen Effekte der ETI auf das Outcome zur Verfügung stehen und dass die Evidenz zu gering ist, um klare Empfehlungen für oder gegen die präklinische Intubation von SHT-Patienten auszusprechen.

\subsubsection{Ertrinkungsunfälle}

Ertrinken ist eine der häufigsten Todesursachen im Alter zwischen ein und 14 Jahren. In der Untersuchung von EICH et al. (2009b) im Rettungsdienstbereich Göttingen machten Ertrinkungsunfälle knapp ein Prozent aller Notfalleinsätze bei Kindern aus.

In der vorliegenden Untersuchung erlitten sieben Kinder (8,5\%) einen Ertrinkungsunfall, woraus in allen Fällen ein Atem-Kreislauf-Stillstand resultierte. Ertrinkungsunfälle stellten in unserem Kollektiv mit gut einem Viertel aller Fälle die zweit-häufigste Ursache für einen Atem-Kreislauf-Stillstand dar. Betroffen waren vier Kleinkinder und drei Schulkinder. Diese Altersgruppen zeigen typischerweise die höchsten Prävalanzen für Ertrinkungsunfälle (EICH et al. (2009b)). Bei sechs dieser Kinder (85,7\%) konnte noch am Notfallort ein Spontankreislauf wiederhergestellt werden. Vier Kinder (66,7\%) konnten in guter neurologischer Verfassung, zwei Kinder mit schweren neurologischen Residuen aus der Klinik entlassen werden.

Durch Ertrinkungsunfälle bedingten Atem-Kreislauf-Stillständen werden in der Literatur bessere Überlebensraten zugeschrieben, als durch andere Ätiologien verursachte (QUAN et al. (1990)). Diese schwanken zwischen 17\% und 70\% (SUOMINEN et al. (2002), YOUNG et al. (2004), SIRBAUGH et al. (1999)). Kinder, die mit stabilen Vitalzeichen in die Klinik eingewiesen werden und nicht komatös sind, haben eine Überlebensrate von beinahe 100\% (KEMP und SIBERT (1991)).

Die Submersionszeit (Anoxiezeit) ist neben dem frühzeitigen Beginn von Basisreanimationsmaßnahmen einer der eindeutig nachgewiesenen prognostisch relevanten Faktoren für das Überleben und das neurologische Outcome (QUAN und KINDER (1992), SUOMINEN et al. (2002)). Von einigen Autoren wird eine Grenze von zehn Minuten formuliert. Wenn die Submersionszeit in relativ warmen Wasser (> $5^{\circ}$ Celsius) mehr als 25 Minuten beträgt, gibt es praktisch keine Überlebenschancen oder die Reanimationsbemühungen münden in einem persistierenden vegetativen Status (apallisches Syndrom) oder Hirntod (SUOMINEN et al. 2002)). 
Einige Autoren sind der Meinung, dass die Qualität der notfallmedizinischen Versorgung das Outcome entscheidend beeinflusst und beinahe-ertrunkene Kinder von einer frühen endotrachealen Intubation profitieren könnten (QUAN und KINDER (1992), SIRBAUGH et al. (1999), SUOMINEN et al. 2002)). Eindeutige Daten, die dies belegen, gibt es jedoch nicht.

\subsection{Die „Pros und Contras“ der präklinischen Intubation von Kindern}

Wie in dem vorangehenden Teil der Diskussion beschrieben, weisen einige Studien auf einen Überlebensvorteil bereits präklinisch intubierter Kinder hin. In einigen internationalen Leitlinien wird die frühe endotracheale Intubation empfohlen. Zur definitiven Sicherung der Atemwege wird die ETI als Goldstandard der Versorgung erachtet (BIARENT et al. (2005), ILCOR (2005)).

Die potenziellen Vorteile der endotrachealen Intubation sind (NOLAN und SOAR (2008)):

(1) Möglichkeit einer kontrollierten invasiven Beatmung mit hohen in- und exspiratorischen Atemwegsdrücken.

(2) Reduzierung der Gefahr von Mageninsufflation und konsekutiver Regurgitation.

(3) Schutz vor pulmonaler Aspiration.

(4) Endobronchiale Absaugung und Medikamentengabe.

(5) Kontinuierliche Herzdruckmassage im Rahmen einer kardiopulmonalen Reanimation.

Auf der anderen Seite birgt die präklinische Intubation potenzielle Risiken und Nachteile anderer Art in sich. Inadäquates Atemwegsmanagement ist einer der wesentlichen Faktoren, welche die präklinische Morbidität und Mortalität beeinflussen (HELM et al. (2006)). Wie bereits ausführlich diskutiert und auch durch die eigenen Ergebnisse aufgezeigt wird, treten Intubationszwischenfälle regelmäßig und in teilweise hoher Zahl auf.

Ein weiterer Faktor, der gegen eine präklinische Intubation bei kritisch erkrankten oder verletzten Kindern sprechen könnte, ist die damit verbundene zeitliche Verzögerung und somit eine mögliche Verlängerung der präklinischen Versorgungszeit („on-scene-time“). SCHMIDT et al. (2002) fanden eine um durchschnittlich 10 Minuten verlängerte Verweildauer am Unfallort pädiatrischer Traumapatienten in Hannover, wenn eine Intubation durchgeführt wurde. In einer retrospektiven Analyse von CUDNIK et al. (2007) an erwachsenen Traumapatienten betrug die durchschnittliche präklinische Versorgungszeit bei Intubierten 10,7 Minuten länger als in der Kontrollgruppe der Nicht-intubierten. In Untersuchungen mit pädiatrischen Patienten konnten GAUSCHE et al. (2000), SUOMINEN 
et al. (2000a) und DIRUSSO et al. (2005) ebenfalls eine signifikant längere „on-scene-time“ in der Intubationsgruppe nachweisen. Ob sich diese Verlängerung auf das Outcome auswirkt, geht aus keiner der Studien hervor.

Die genannten Untersuchungen über den Einfluss der präklinischen endotrachealen Intubation von Kindern auf das Outcome liefern kontroverse Ergebnisse. Diese Kontroversen deuten darauf hin, dass es nicht die Intervention per se zu sein scheint, die das Outcome beeinflusst, sondern dass dieses auch maßgeblich durch andere Variablen beeinflusst wird. Dazu zählen strukturelle Gegebenheiten, z.B. Organisation des Rettungssystems, präklinische Versorgungszeiten sowie die Fähigkeiten und Kompetenzen der Rettungskräfte (UMMENHOFER und SCHEIDEGGER (2002)). Obwohl ein direkter Vergleich zwischen den verschiedenen Rettungssystemen aus vielen Gründen kaum möglich ist, fällt auf, dass die Überlebensraten in US-amerikanischen Untersuchungen insgesamt eher schlechter sind und dass die ETI dort keinen Benefit mit sich zu bringen scheint, verglichen mit der BeutelMasken-Beatmung. In den europäischen Studien bzw. den Untersuchungen mit Notarztbeteiligung wird der ETI überwiegend ein mortalitäts-senkender Effekt zugeschrieben und eher die Meinung vertreten, dass die ETI ein sicheres und effektives Verfahren ist, wenn sie von qualifizierten Notärzten durchgeführt wird (MEYER et al. (2000), MACKAY et al. (2001), MARTINON et al. (2010)).

Bisher gibt es keine evidenz-basierten Empfehlungen für die präklinische Intubation. In einem kürzlich veröffentlichten Cochrane-Review folgerten LECKY et al. (2008, S. 2): „Evidence base provides no imperative to extend the practice of prehospital intubation in urban systems in pediatric patients."

Auf der anderen Seite existieren für zahlreiche medizinische Interventionen keine robusten Daten und evidenz-basierte Empfehlungen, wie z.B. für die intravenöse Gabe von Adrenalin beim Herz-Kreislaufstillstand. Die Frage, die sich hinsichtlich der ETI bei kritisch kranken oder verletzten Kindern stellt, ist nicht ob, sondern wann, wo und von wem die ETI am besten durchgeführt werden sollte (EICH et al. (2010)).

Unsere Daten sprechen dafür, dass die präklinische Intubation von Kindern indiziert ist, wenn (1.) die Atemwege und/oder die Atmung ernsthaft kompromittiert oder bedroht sind, (2.) der Transportmodus und die Dauer des Transportes einen frühzeitig gesicherten Atemweg erfordern (z.B. Lufttransport) und (3.) ein im pädiatrischen Atemwegsmanagement versierter Notarzt und der Einsatz entsprechender Narkotika und Muskelrelaxanzien verfügbar ist. 
Die Indikation zur Intubation von Kindern und die Wahl des richtigen Zeitpunktes hängen daher von verschiedenen Faktoren ab. Insbesondere, wenn nur kurze Transportwege in die Klinik vorliegen, sollten sich weniger Geübte auf Basismaßnahmen oder ggf. auf alternative Atemwegshilfen (z.B. Larynxmaske, etc.) beschränken. Oberste Prämisse jeglichen Atemwegsmanagements muss es sein, größtmögliche Patientensicherheit und bestmögliche Effektivität von Oxygenierung und Ventilation zu gewährleisten.

\subsection{Alternativen zur endotrachealen Intubation}

SCOTT (1986, S. 157) stellte folgende These auf: „Patients do not die from failure to intubate; they die from failure to stop trying to intubate or from undiagnosed oesophageal intubation."

Ein Patient stirbt nicht, weil ihm ein endotrachealer Intubationsversuch vorenthalten wird, sondern weil es misslingt die Lungen zu ventilieren. Dies kann auch verursacht werden durch eine unerkannte ösophageale Fehllage eines Endotrachealtubus oder durch eine Hypoxämie nach prolongierten Intubationsversuchen (VON GOEDECKE et al. (2007)).

Beim Vorliegen eines schwierigen Atemweges oder einer unerwartet schwierigen Intubation (sog. „Cannot intubate, cannot ventilate“-Situation) ist es erforderlich eine Strategie als Alternative zur endotrachealen Intubation vorzuhalten, um eine Hypoxie zu vermeiden (BYHAHN et al. (2007), MEYBURG et al. (2009), WALKER und ELLWOOD (2009)). Dafür kann zum einen auf die Beutel-Masken-Beatmung zurückgegriffen werden oder es können supraglottische Atemwegshilfen (SGA) zur Anwendung kommen. Als Ultima Ratio muss die Koniotomie in Erwägung gezogen werden (COTE und HARTNICK (2009)).

Für den in der pädiatrischen Intubation Untrainierten ist es akzeptabel und wahrscheinlich ebenso effektiv die Beutel-Masken-Beatmung oder alternative Atemwegshilfen als primäres Hilfsmittel zur Sicherung des Atemweges bzw. zur Gewährleistung von Oxygenierung und Ventilation einzusetzen (NICOLAI (2008)). In der notfallmedizinischen Praxis am weitesten verbreitet ist aufgrund seiner einfachen Anwendung und schnellen Verfügbarkeit die BeutelMasken-Ventilation (BMV). Diese kann jedoch nicht dieselbe Qualität der respiratorischen Unterstützung wie die Intubation erreichen. $\mathrm{Zu}$ den Nachteilen und Komplikationsmöglichkeiten der Maskenbeatmung zählen der ungesicherte Luftweg und damit verbunden die Gefahr der Mageninsufflation mit konsekutiver Regurgitation und möglicher Aspiration (VON GOEDECKE et al. (2006), NEUBERT (2007)). Ein weiteres Problem der Beatmung mittels Gesichtsmaske und Beutel ist die Gewährleistung der 
Systemdichtigkeit mit der möglichen Gefahr einer Hypoventilation und Hypoxie (LANGERON et al. (2000)). Jedoch kann die BMV für einen kurzen Zeitraum ebenso effektiv sein wie die endotracheale Intubation (ILCOR (2005)-Empfehlungen: Klasse-IIaEmpfehlung). Wie in der GAUSCHE et al. (2000)-Studie berichtet, gab es im Vergleich zur Intubationsgruppe keinen Unterschied in der Oxygenierung und der Häufigkeit an Komplikationen bei Kindern, die mit Beutel-Maske beatmet wurden.

DOERGES et al. (2003b), verglichen verschiedene Strategien zur Ventilation des apnoeischen, nicht-präoxygenierten Patienten miteinander. Sie konnten zeigen, dass die Beatmung durch Rettungsassistenten mit Beutel und Maske die einfachste und erfolgreichste Strategie war. Einige Autoren sind somit der Ansicht, dass in der Präklinik Tätige, die keine profunden Erfahrungen im Atemwegsmanagement haben, die Beutel-Masken-Beatmung als erstes Mittel der Wahl zur Notfallbeatmung bei Patienten mit Atem- oder HerzKreislaufstillstand einsetzen oder aber als „Rückzugstrategie“ nach erfolglosem ersten Intubationsversuch anwenden sollten (VON GOEDECKE et al. (2006)).

In den letzten Jahren sind eine Reihe alternativer Atemwegshilfen in verschiedenen Variationen und Modifikationen entwickelt worden. SGA sind in der präklinischen Anwendung evaluiert, einfach in der Anwendung und gekennzeichnet durch eine hohe Erfolgs- bei niedriger Komplikationsrate (DEAKIN et al. (2005), GENZWUERKER et al. (2006)). Die Nachteile der Beutel-Masken Beatmung wie die mangelnde Systemdichtigkeit, zu geringe Tidalvolumina und die größere Gefahr für Mageninsufflation mit der Folgegefahr der Aspiration, werden durch die SGA erheblich reduziert. Für den Einsatz im Bereich der präklinischen Notfallmedizin stellen sie daher praktikable und wertvolle Hilfsmittel dar (BIARENT et al. (2005), TIMMERMANN und RUSSO (2007)). Die zur Verfügung stehenden präklinischen Studien über SGA basieren auf Untersuchungen bei Erwachsenen.

Die bekannteste und am besten untersuchte SGA ist die Larynxmaske. Sie ist nicht nur beim Erwachsenen, sondern auch beim Kind mit schwierigem Atemweg ein effektives Instrument zum Offenhalten der Atemwege und im innerklinischen Bereich weltweit seit über 20 Jahren etabliert (GENZWUERKER et al. (2006), WEISS et al. (2009)). GUYETTE et al. (2007) sowie CHEN und HSIAO (2008) konnten in prospektiv randomisierten Studien eines simulierten pädiatrischen Kreislaufstillstandes am Phantom zeigen, dass der Einsatz der Larynxmaske durch Medizinstudenten bzw. Paramedics im Vergleich zum Endotrachealtubus $\mathrm{zu}$ einer rascheren Etablierung effektiver Ventilationen und $\mathrm{zu}$ weniger Komplikationen führte. In den „Consensus on Science and Treatment Recommendations“ der ILCOR (2005) 
wurde der primären Anwendung der Larynxmaske beim Kind in der präklinischen Notfallmedizin eine Klasse-III-Empfehlung, bei Scheitern der Intubation eine Klasse-IIbEmpfehlung ausgesprochen.

Auch bei einem Säugling in dieser Untersuchung gelang es, mit Hilfe einer Larynxmaske nach gescheiterten Intubationsversuchen eine adäquate Ventilation zu erzielen.

Die Fastrach ${ }^{\circledR}$-Intubationslarynxmaske (LMA Deutschland GmbH, Bonn) ist ein wertvolles Hilfsmittel im Management des schwierigen Atemweges in der Anästhesie und wurde auch im Rahmen eines schwierigen Atemwegsmanagements im präklinischen Bereich evaluiert (TIMMERMANN et al. (2007a)). Eine effektive Ventilation ist mit Hilfe der Intubationslarynxmaske schnell und sicher zu erzielen. In einem hohen Prozentsatz nach erfolgloser konventioneller Laryngoskopie gelingt es einen Endotrachealtubus blind via Intubationslarynxmaske zu platzieren. Für die Anwendung im pädiatrischen Atemwegsmanagement ist sie nur eingeschränkt einsetzbar, da die kleinste verfügbare Größe (3) ein Körpergewicht von mindestens 25-30 kg erfordert (WEISS et al. 2001b)).

Der Larynxtubus (VBM Medizintechnik GmbH, Sulz am Neckar, Deutschland) und seine Weiterentwicklungen $\left(\right.$ LTS $^{\circledR}$, LTS II ${ }^{\circledR}$ und LTS-D ${ }^{\circledR}$ ) konnten sich seit seiner Einführung im Jahr 1999 in der Notfallmedizin etablieren (DOERGES et al. (2003a)). Die sichere und effektive Ventilation auch unter Reanimationssituationen wurde in präklinischen Studien nachgewiesen (WIESE et al. (2008), WIESE et al. (2009)). Aufgrund der Verfügbarkeit entsprechender Größen, kann der Larynxtubus für alle Altersgruppen eingesetzt werden. Wie SCHELLER et al. (2009) in einer Fallserie berichten, wurde der LTS II bei zehn Neugeborenen und Säuglingen erfolgreich angewendet, nachdem Versuche der ETI und der konventionellen Beutel-Masken-Beatmung misslangen. Die Autoren empfahlen den LTS II als primäres Hilfsmittel zur Sicherung der Atemwege in Notfallsituationen von Neugeborenen und Säuglingen, wenn die Intubation nicht möglich oder zu zeitaufwendig ist.

Der Einsatz des Combitubus (Combitube ${ }^{\circledR}$; Covidien Deutschland GmbH, Neustadt/Donau), einem ösophagotrachealen Doppellumentubus, ist aufgrund der zur Verfügung stehenden Größen nur bei größeren Kindern (> $120 \mathrm{~cm}$ ) und Adoleszenten möglich.

Supraglottische Atemwegshilfen bieten eine probate Alternative im Atemwegsmanagement bei erfolgloser ETI. Für denjenigen Anwender, der keine profunden Intubationskenntnisse besitzt, sind sie als primäres Hilfsmittel zur Sicherung der Atemwege und der Ventilation oftmals eine bessere Option als ein endotrachealer Intubationsversuch (NOLAN und SOAR 
(2008), STRAUSS und BECKE (2010)). Jedoch setzt auch die Anwendung von SGA in Notfallsituationen Vertrautheit und klinische Erfahrung mit dem entsprechenden Hilfsmittel voraus (LOPEZ-GIL et al. (1996), PAAL et al. (2010a)).

Eine Alternative zur konventionellen endotrachealen Intubation ist die seit wenigen Jahren im klinischen Bereich eingeführte videoassistierte Laryngoskopie (DOHERTY et al. (2009)). In klinischen Untersuchungen und Fallberichten hat sich gezeigt, dass sich die laryngoskopische Sicht im Vergleich zur konventionellen direkten Laryngoskopie deutlich verbessern lässt (COOPER RM et al. (2005), MAHARAJ et al. (2008)). In vielen Fällen einer schwierigen oder misslungenen konventionellen Intubation gelingt mit Hilfe der indirekten Videolaryngoskopie die erfolgreiche tracheale Platzierung eines Tubus unter Sicht (NICOLAI und HOFFMANN (2009)). Auch im pädiatrischen Bereich wurde dieses Verfahren untersucht und validiert (TREVISANUTO et al. (2006), REDEL et al. (2009)). Einige Hersteller bieten portable Geräte an, die grundsätzliche für den präklinischen Bereich geeignet sind. Inwieweit die video-assistierte Laryngoskopie die Bedingungen der präklinischen Intubation von Kindern verbessern kann, muss Gegenstand zukünftiger Studien sein.

\subsection{Strategien zur Aus-, Weiter- und Fortbildung im pädiatrischen Atemwegsmanagement}

Kindernotfälle sind oftmals charakterisiert durch die Trias zeitkritisch, schwierig und selten (EICH et al. (2007). Dies kann - vor allem bei nicht speziell pädiatrisch geschulten Notfallmedizinern - zu Angst und Unsicherheit und neben der fehlenden klinischen Expertise im Umgang mit Kindernotfällen zu einer Minderung der notärztlichen Versorgungsqualität führen (GRIES et al. (2006)). Bei dringender Notwendigkeit einer Öffnung und Sicherung des kindlichen Atemweges können daraus dramatische Konsequenzen resultieren.

Die endotracheale Intubation erfordert komplexe psychomotorische Fertigkeiten, die ein adäquates Erlernen und eine ausreichende kontinuierlich-praktische Erfahrung voraussetzt (DEAKIN et al. (2008)). KONRAD et al. (1998) kamen in einer Untersuchung an Weiterbildungsassistenten der Anästhesie im ersten Jahr zu dem Ergebnis, dass unter OPBedingungen bei Erwachsenen zum Erreichen einer 90-prozentigen Erfolgsrate im ersten Intubationsversuch im Median 57 Intubationen notwendig waren. Wie die Realität hinsichtlich praktischer Intubationserfahrung im deutschen Notarztwesen aussieht, konnte in einer Umfrage von TIMMERMANN et al. (2007b) unter Notärzten in Norddeutschland 
demonstriert werden. 20\% aller Befragten haben insgesamt weniger als 20 Intubationen unter Aufsicht durchgeführt, bevor sie im Notarztdienst eingesetzt wurden. Man kann somit annehmen, dass ein großer Teil der Notärzte in Deutschland keine ausreichende Erfahrung in der präklinischen Intubation von Kindern hat.

Wie die vorliegende Arbeit zeigt, ist die präklinische Intubation von Kindern bezogen auf den individuellen Notarzt eine seltene Maßnahme. Auch auf die Gesamtheit aller Notarzteinsätze betrachtet (Erwachsene und Kinder), kommen Notärzte nur auf geringe Intubationszahlen. GRIES et al. (2006) berechneten, dass ein Notarzt statistisch lediglich 0,7 Intubationen pro Monat durchführt. Profunde Fertigkeiten in der Intubation von Kindern können daher in präklinischer Tätigkeit allein weder erworben noch aufrecht erhalten werden. Um eine adäquate Routine in der Durchführung dieser Maßnahme zu erlangen, ist ein kontinuierliches, praktisches Training unter reproduzierbaren innerklinischen Bedingungen erforderlich (RICHARD et al. (2006), TIMMERMANN et al. (2006)).

Die Erfolgsraten von Interventionen im Atemwegsmanagement sind im Wesentlichen abhängig von den drei Faktoren Ausbildung, kontinuierliches Training und klinische Routine. Adäquates Training für Personal, das mit kritischen pädiatrischen Notfällen konfrontiert wird, ist von entscheidender Bedeutung (VON ELM et al. (2009)). Dazu können auch strukturierte Kurse im Atemwegsmanagement beitragen, die idealerweise aus Vorträgen, Übungsstationen und simulator-unterstützten Szenarien bestehen und damit besonders effektiv das Lernverhalten beeinflussen (RUSSO et al. (2007)).

Zur Erlangung und Aufrechterhaltung der Fertigkeiten im Umgang mit pädiatrischen Notfällen sollten Notärzte sowohl klinische Erfahrungen im Bereich der Kinderanästhesie und Kinderintensivmedizin erwerben als auch in regelmäßigen Abständen an zertifizierten Kursen wie z.B. dem European Paediatric Life Support (EPLS), Atemwegsmanagementkursen oder simulator-basierten Kursen (z.B. PaedSim) teilnehmen (PHILLIPS et al. (2000), EPPICH et al. (2006), EICH et al. (2007), SCHMID et al. (2009)). 


\subsection{Einschränkungen der Untersuchung}

Als grundsätzliche Limitation dieser Studie muss berücksichtigt werden, dass es sich um eine prospektive Observationsstudie handelt, die somit keine Kontrollgruppe oder Randomisierung beinhaltet.

Das Ein-Zentrum-Studiendesign schränkt die Vergleichbarkeit mit anderen Untersuchungen ein. Aufgrund des überdurchschnittlichen Ausbildungsstands der Studienärzte können die Ergebnisse nicht uneingeschränkt auf andere Studienkollektive übertragen werden.

Schließlich sind die gewonnenen Daten über die Atemwegscharakteristika durch die Studiennotärzte selbst-berichtet und unterliegen somit dem Risiko eines systematischen „reporting bias“. 


\subsection{Schlussfolgerungen}

Die Haupterkenntnis der vorliegenden Untersuchung ist, dass anästhesiologisch weitergebildete Notärzte die präklinische endotracheale Intubation von Kindern mit einer hohen Erfolgsrate durchführten (98,3\%). Die Rate an intubations-assoziierten Komplikationen bei den Notärzten mit anästhesiologischer Weiterbildung (Gruppe 1) war relativ gering. Die Outcome-Parameter „Überleben bis zur Krankenhausentlassung“ und „gutes neurologisches Outcome“" waren im Vergleich zu anderen Untersuchungen bemerkenswert hoch.

Unsere Ergebnisse sprechen dafür, dass die prähospitale ETI von Kindern angezeigt ist, wenn die Atemwege oder die Atmung ernsthaft kompromittiert oder bedroht sind, wenn der Transportmodus und/oder die Transportdauer eine frühzeitige und definitive Sicherung der Atemwege nahelegen (z.B. Lufttransport) und unterstützen die These, dass die präklinische Intubation von Kindern zum therapeutischen Repertoire adäquat trainierter und erfahrener Notärzte gehören sollte.

Insgesamt ist die präklinische endotracheale Intubation von Kindern eine relativ selten durchgeführte Prozedur. Adäquate manuelle Fertigkeiten und fortlaufende praktische Erfahrung in der Intubation von Kindern sind essentielle Voraussetzungen und müssen in der innerklinischen Praxis erworben und aufrechterhalten werden.

Jedoch können diese hohen Anforderungen nicht von allen Notärzten erfüllt werden. Jeder Notarzt sollte seine eigenen Kompetenzen und Fähigkeiten in Bezug auf pädiatrisches Atemwegsmanagement kennen und eine geeignete Atemwegsstrategie bereit halten, inklusive der Verwendung alternativer Techniken zur effektiven Oxygenierung und Ventilation.

Abschließend lässt sich feststellen, dass die präklinische Intubation von Kindern weder unkritisch und uneingeschränkt empfohlen noch pauschal abgelehnt werden kann. Sie muss bezüglich Indikation, Zeitpunkt und Ort sorgfältig abgewogen werden. Eine optimale präklinische Versorgung lebensbedrohlich erkrankter oder verletzter Kinder erfordert eine differenzierte und individualisierte Vorgehensweise. 


\section{Zusammenfassung}

ZIEL unserer Untersuchung war es, detaillierte Daten über die Charakteristika präklinischer endotrachealer Intubationen (ETI) von Kindern und deren Outcome zu erhalten, wenn sie von anästhesiologisch weitergebildeten Notärzten durchgeführt werden.

MATERIAL UND METHODIK: Wir führten eine prospektive Observationsstudie in einem Acht-Jahres-Zeitraum an den Notarztstandorten des Zentrums Anaesthesiologie, Rettungsund Intensivmedizin (ZARI) der Universitätsmedizin Göttingen durch. In die Untersuchung eingeschlossen wurden alle Kinder im Alter von 0 bis 14 Jahren, die präklinisch endotracheal intubiert wurden. Die ETI wurden entweder von Studiennotärzten des ZARI durchgeführt (Gruppe 1) oder von primär versorgenden Notärzten (Gruppe 2), mit sekundärer Übernahme des Patienten durch die Studienärzte.

ERGEBNISSE: 82 von insgesamt 2040 Kindernotfällen (4,0\%) erfüllten die Einschlusskriterien (58 in Gruppe 1, 24 in Gruppe 2). Die häufigsten Diagnosen waren Traumata (50,0\%) Krampfanfälle $(13,4 \%)$ und der plötzliche Kindstod $(12,2)$. Mit Ausnahme eines Säuglings, welcher schließlich mit einer Larynxmaske versorgt wurde, konnten alle Kinder der Gruppe 1 erfolgreich intubiert werden (98,3\%). Im Vergleich zu den älteren Kindern gab es bei Säuglingen eine höhere Inzidenz einer erschwerten laryngoskopischen Sicht (Cormack-Lehane-Grad $\geq 3$ ) und einer schwierigen Intubationen (je 23,1\%) sowie signifikant geringere Erfolgsraten im ersten Intubationsversuch $(\mathrm{p}=0,04)$. Intubationszwischenfälle traten in 25,0\% der Patienten der Gruppe 2 und 6,0\% der Fälle der Gruppe 1 auf. Von allen 82 Kindern überlebten 71 (86,6\%) bis zur Krankenhausaufnahme und $63(76,8 \%)$ bis zur Entlassung. Von den 63 überlebenden Kindern zeigten 54 (85,7\%) ein gutes oder unverändertes neurologisches Outcome. Überlebensrate und neurologisches Outcome in der Altersgruppe der Säuglinge waren signifikant schlechter als bei Klein- und Schulkindern $(\mathrm{p}<0,001)$. Im Durchschnitt führte ein Notarzt eine ETI bei einem Kind alle drei Jahre, bei einem Kleinkind alle acht Jahre und bei einem Säugling alle 13 Jahre durch.

SCHLUSSFOLGERUNGEN: Anästhesiologisch weitergebildete Notärzte führten die präklinische ETI bei Kindern mit einer hohen Erfolgsrate durch. Überlebensrate und neurologisches Outcome waren bemerkenswert besser als in vergleichbaren Studien. Die frühe und versierte ETI kann für kritisch kranke und verletzte Kinder einen individuellen Versorgungs- und Überlebensvorteil mit sich bringen. 


\section{Anhang}

\subsection{Die RED $^{\circledR}$-Eingabemaske zu den Charakteristika des Atemwegsmanagements}

Intubation oder Alternativen

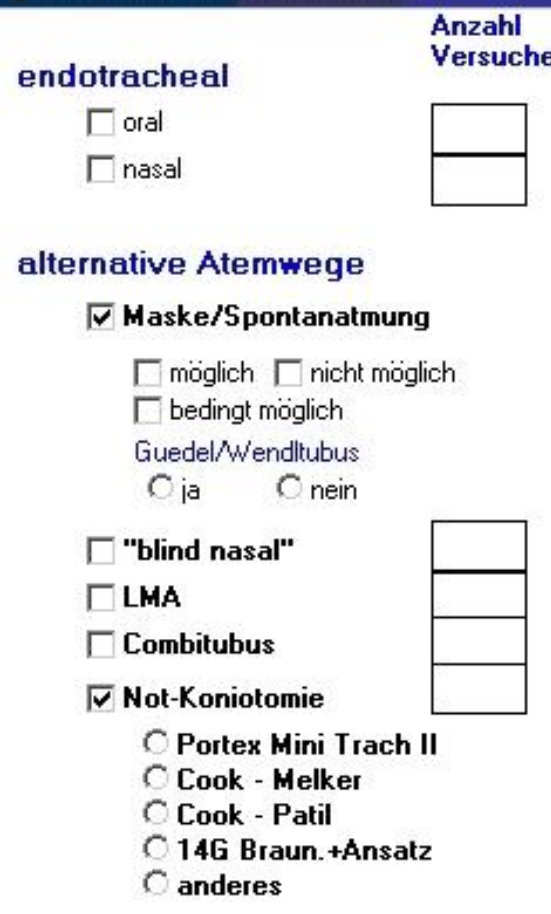

$\Gamma$ sonstige Sicherung der Atemwege

$$
\begin{aligned}
& \text { mit Erfolg? } \\
& C_{\text {ja }} C_{\text {nein }} \\
& c_{\text {ia }} C_{\text {nein }}
\end{aligned}
$$

$$
\text { (1) }
$$

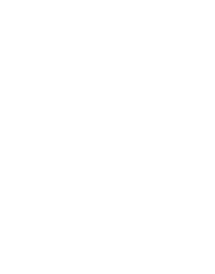$$
\mathrm{C}_{\mathrm{ja}} \mathrm{C} \text { nein }
$$$$
\text { Cia } C_{\text {nein }}
$$$$
C \text { ja } C \text { nein }
$$$$
O_{\text {ja }} \mathrm{O}_{\text {nein }} \underset{\begin{array}{r}
\text { nicht } \\
\text { beurteilbar }
\end{array}}{ }
$$

\section{Cormack/Lehane}

Score

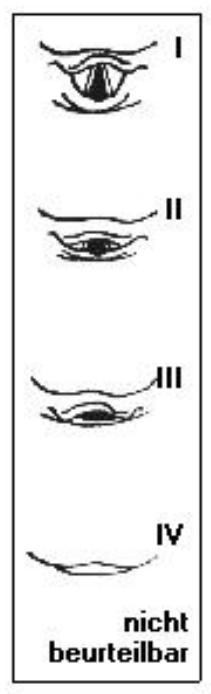

Bemerkungen:
DK

Abbrechen $\sqrt{\checkmark}$ schwierige Intubation subjektive Einschätzung

$\Gamma$ anatom. Besonderheit

$$
\Gamma \text { große Zunge }
$$$$
\Gamma \text { kleine Mundöffnung }
$$

$\Gamma$ traumat. Behinderung

$\Gamma$ Lage des Patienten

$\Gamma$ sonstiges

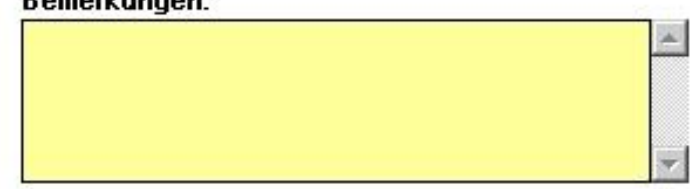




\subsection{Abbildungsverzeichnis}

Abbildung 1: Chain-of-Survival aus ILCOR (2000), S. 345 ................................................. 1

Abbildung 2: Laryngoskopie mit geradem Laryngoskopspatel aus ILCOR (2000), S. 354 ....... 4

Abbildung 3: Cormack-Lehane-Skala aus SAMSOON und YOUNG (1987), S. 488............... 13

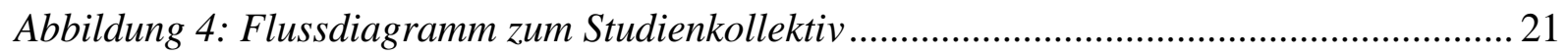

Abbildung 5: Balkendiagramm der Altersverteilung ......................................................... 22

Abbildung 6: Balkendiagramm der Altersgruppen ............................................................. 22

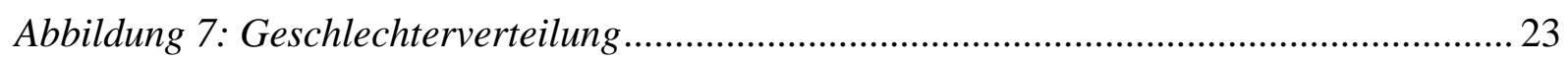

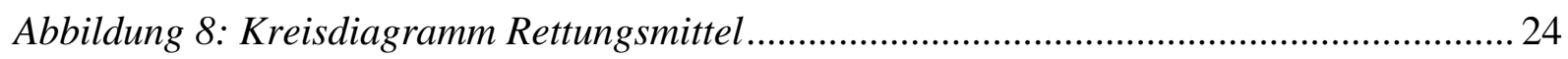

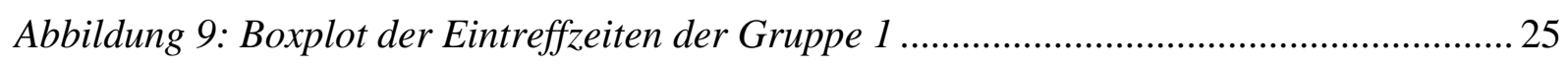

Abbildung 10: Boxplot der präklinischen Versorgungsintervalle der Gruppe 1 .................... 26

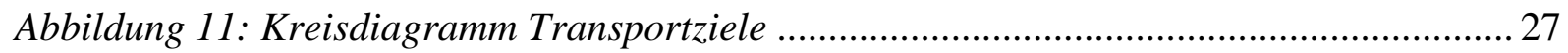

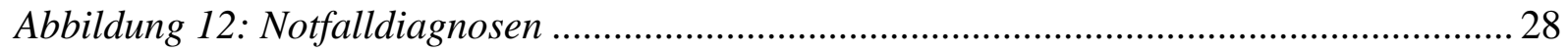

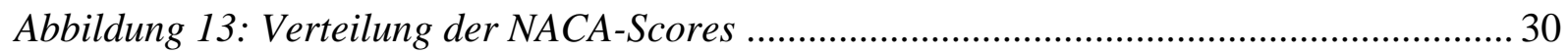

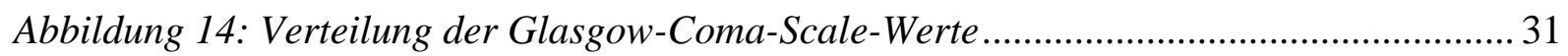

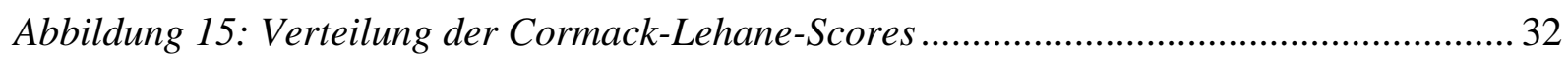

Abbildung 16: Inzidenz der schwierigen Intubation ........................................................... 33

Abbildung 17: Kumulative Erfolgsrate (Gruppe 1) ............................................................... 34

Abbildung 18: Erfolgsraten der Intubationsversuche (Gruppe 1, $n=58$ ) ............................ 35

Abbildung 19: Häufigkeit der Intubationszwischenfälle ........................................................ 36

Abbildung 20: Arten der Intubationszwischenfälle ................................................................ 36

Abbildung 21: Boxplot der Beatmungsdauer nach Altersgruppen ....................................... 38

Abbildung 22: Boxplot der Intensivbehandlungsdauer nach Altersgruppen ........................... 38

Abbildung 23: Boxplot der Krankenhausaufenthaltsdauer nach Altersgruppen ..................... 39

Abbildung 24: Häufigkeit von Atem-Kreislauf-Stillständen nach Altersgruppen ..................... 41

Abbildung 25: Ätiologie von pädiatrischen Atem-Kreislauf-Stillständen ................................ 42

Abbildung 26: Häufigkeitsverteilung der Traumanotfälle nach Altersgruppen ...................... 44

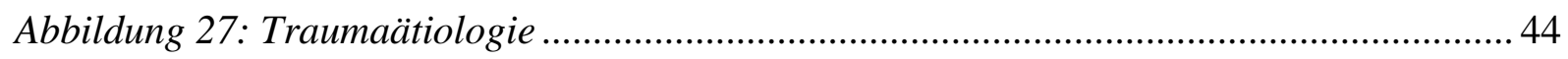

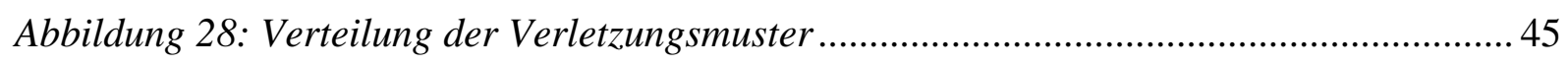

Abbildung 29: Kreisdiagramm Ort des Ertrinkens ............................................................. 48 


\subsection{Tabellenverzeichnis}

Tabelle 1: Definition der laryngoskopischen Sicht nach CORMACK und LEHANE (1984) .. 13

Tabelle 2: Modifizierter NACA-Index nach Tryba ............................................................... 14

Tabelle 3: Glasgow-Coma-Scale für Erwachsene und Kinder > 5 Jahre ............................... 16

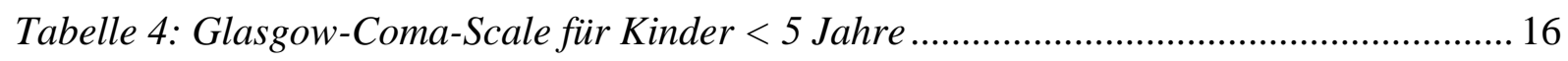

Tabelle 5: Paediatric Cerebral Performance Category Scale (PCPC) .................................. 18

Tabelle 6: Geschlechterverteilung in Abhängigkeit der Altersgruppen .................................. 23

Tabelle 7: Art des Rettungsmittels nach Altersgruppen ....................................................... 24

Tabelle 8: Art des Rettungsmittels nach Behandlungsgruppen............................................... 24

Tabelle 9: Häufigkeit der Einweisungsdiagnosen in Abhängigkeit von der Altersgruppe ...... 28

Tabelle 10: Diagnosen nach Art des Rettungsmittels ............................................................ 29

Tabelle 11: Verteilung der NACA-Scores nach Altersgruppen.............................................. 30

Tabelle 12: Evaluierung der Cormack-Lehane-Scores durch die Studienärzte (Gruppe 1).... 32

Tabelle 13: Inzidenz einer schwierigen Intubation (Gruppe 1) ............................................ 33

Tabelle 14: Ursachen einer schwierigen Intubation ............................................................. 33

Tabelle 15: Analyse der Intubationsversuche in der Gruppe 1 ............................................. 35

Tabelle 16: Überlebensrate und Outcome in Korrelation zu den drei Altersgruppen............. 37

Tabelle 17: Überlebensrate und Outcome nach Behandlungsgruppen .................................. 37

Tabelle 18: Paediatric Cerebral Perfomance Categories (PCPC) ........................................ 40

Tabelle 19: Neurologisches Outcome differenziert nach Behandlungsgruppen ...................... 40

Tabelle 20: Charakteristika und Begleitumstände bei Atem-Kreislauf-Stillstand ................... 41

Tabelle 21: Ätiologie von Atem-Kreislauf-Stillständen nach Alters gruppen ........................... 42

Tabelle 22: Outcome der Kinder mit Atem-Kreislauf-Stillstand........................................... 43

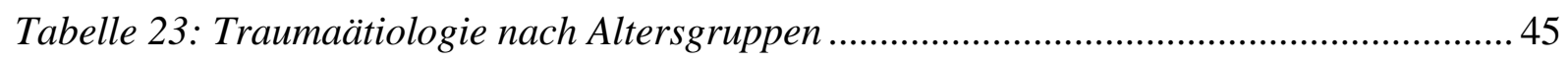

Tabelle 24: Verletzungsmuster nach Altersgruppen ........................................................... 46

Tabelle 25: NACA- und GCS-Scores der traumatisierten Kinder ......................................... 46

Tabelle 26: Präklinische Versorgungsintervalle beim kindlichen Trauma ............................ 46

Tabelle 27: Outcome pädiatrischer Traumapatienten ......................................................... 47

Tabelle 28: Überlebensrate und Outcome von Kindern nach Ertrinkungsunfall .................... 49

Tabelle 29: Submersionszeiten und Outcome ...................................................................... 49 


\section{Literaturverzeichnis}

Adewale L (2009): Anatomy and assessment of the pediatric airway. Paediatr Anaesth 19 Suppl 1, 1-8

Albrech M, Berner J, Altemeyer KH (2000): Kindernotfälle im Luftrettungsdienst. Notfall Rettungsmed $\underline{3}, 156-169$

Ärztekammer Niedersachsen: Weiterbildungsordnung der Ärztekammer Niedersachsen. AEKN, Hannover 2004

Babl FE, Vinci RJ, Bauchner H, Mottley L (2001): Pediatric pre-hospital advanced life support care in an urban setting. Pediatr Emerg Care 17(1), 5-9

Becke K, Schmidt J (2007): Das aspirationsgefährdete Kind - Rapid Sequence Induction im Kindesalter. Anasthesiol Intensivmed Notfallmed Schmerzther 42(9), 624-631

Berg MD, Nadkarni VM, Berg RA (2008): Cardiopulmonary resuscitation in children. Curr Opin Crit Care 14(3), 254-260

Biarent D, Bingham R, Richmond S, Maconochie I, Wyllie J, Simpson S, Nunez AR, Zideman D (2005): European Resuscitation Council guidelines for resuscitation 2005. Section 6. Paediatric life support. Resuscitation $\underline{67}$ Suppl 1, 97-133

Boswell WC, McElveen N, Sharp M, Boyd CR, Frantz EI (1995): Analysis of prehospital pediatric and adult intubation. Air Med J 14(3), 125-128

Brain Trauma Foundation (2007): Guidelines for the management of severe traumatic brain injury. J Neurotrauma 24 Suppl 1, 1-106

Brownstein D, Shugerman R, Cummings P, Rivara F, Copass M (1996): Prehospital endotracheal intubation of children by paramedics. Ann Emerg Med 28(1), 34-39

Burton JH (2006): Out-of-hospital endotracheal intubation: half empty or half full? Ann Emerg Med 47(6), 542-544 
Byhahn C, Meininger D, Walcher F, Hofstetter C, Zwissler B (2007): Prehospital emergency endotracheal intubation using the Bonfils intubation fiberscope. Eur J Emerg Med 14(1), 4346

Chapman SM, Grocott MP, Franck LS (2010): Systematic review of paediatric alert criteria for identifying hospitalised children at risk of critical deterioration. Intensive Care Med $\underline{36}(4)$, 600-611

Chen L, Hsiao AL (2008): Randomized trial of endotracheal tube versus laryngeal mask airway in simulated prehospital pediatric arrest. Pediatrics 122(2), 294-297

Cooper A, DiScala C, Foltin G, Tunik M, Markenson D, Welborn C (2001): Prehospital endotracheal intubation for severe head injury in children: a reappraisal. Semin Pediatr Surg $\underline{10}(1), 3-6$

Cooper RM, Pacey JA, Bishop MJ, McCluskey SA (2005): Early clinical experience with a new videolaryngoscope (GlideScope) in 728 patients. Can J Anaesth $\underline{52}(2), 191-198$

Cormack RS, Lehane J (1984): Difficult tracheal intubation in obstetrics. Anaesthesia $\underline{39}(11), 1105-1111$

Cote CJ, Hartnick CJ (2009): Pediatric transtracheal and cricothyrotomy airway devices for emergency use: which are appropriate for infants and children? Paediatr Anaesth 19 Suppl 1, $66-76$

Cudnik MT, Newgard CD, Wang H, Bangs C, Herringtion R (2007): Endotracheal intubation increases out-of-hospital time in trauma patients. Prehosp Emerg Care 11(2), 224-229

Cummins RO, Hazinski MF (2000): Guidelines based on the principle 'First, do no harm'. New guidelines on tracheal tube confirmation and prevention of dislodgment. Resuscitation $\underline{46}(1-3), 443-447$

Deakin CD (2000): Anaesthetists are best people to provide pre-hospital airway management. BMJ 2000(320), 1006 
Deakin CD, Peters R, Tomlinson P, Cassidy M (2005): Securing the prehospital airway: a comparison of laryngeal mask insertion and endotracheal intubation by UK paramedics. Emerg Med J 22 (1), 64-67

Deakin CD, Clarke T, Nolan J, Zideman D (2008): A Critical Reassessment of Ambulance Service Airway Management in Pre-Hospital Care. JRCALC Airway Working Group. http://www.britishparamedic.org/Airway_Management_Document.doc, 1-30

Dietz HG, Nicolai T (2005): Das Dr. von Haunersche Kinderspital. Ein notfallmedizinisches Zentrum. Notfall Rettungsmed $\underline{8}(1), 60-66$

DiRusso SM, Sullivan T, Risucci D, Nealon P, Slim M (2005): Intubation of pediatric trauma patients in the field: predictor of negative outcome despite risk stratification. J Trauma $\underline{59}(1)$, $84-90$

Doerges V, Ocker H, Wenzel V, Steinfath M, Gerlach K (2003a): The Laryngeal Tube S: a modified simple airway device. Anesth Analg $\underline{96}(2), 618-621$

Doerges V, Wenzel V, Knacke P, Gerlach K (2003b): Comparison of different airway management strategies to ventilate apneic, nonpreoxygenated patients. Crit Care Med 31(3), 800-804

Doherty JS, Froom SR, Gildersleve CD (2009): Pediatric laryngoscopes and intubation aids old and new. Paediatr Anaesth $\underline{19}$ Suppl 1, 30-37

Doran JV, Tortella BJ, Drivet WJ, Lavery RF (1995): Factors influencing successful intubation in the prehospital setting. Prehosp Disaster Med 10(4), 259-264

Dupanovic M, Fox H, Kovac A (2010): Management of the airway in multitrauma. Curr Opin Anaesthesiol 23(2), 276-282

Easley RB, Segeleon JE, Haun SE, Tobias JD (2000): Prospective study of airway management of children requiring endotracheal intubation before admission to a pediatric intensive care unit. Crit Care Med 28(6), 2058-2063 
Ehrlich PF, Seidman PS, Atallah O, Haque A, Helmkamp J (2004): Endotracheal intubations in rural pediatric trauma patients. J Pediatr Surg $\underline{39}(9)$, 1376-1380

Eich C, Timmermann A, Russo SG, Nickel EA, McFadzean J, Rowney D, Schwarz SK (2007): Simulator-based training in paediatric anaesthesia and emergency medicine - thrills, skills and attitudes. Br J Anaesth $\underline{98(4), 417-419}$

Eich C, Roessler M, Timmermann A, Heuer JF, Gentkow U, Albrecht B, Russo SG (2009a): Präklinische Kindernotfälle: Notärztliche Wahrnehmung und Einschätzung. Anaesthesist $\underline{58}(9), 876-883$

Eich C, Russo SG, Heuer JF, Timmermann A, Gentkow U, Quintel M, Roessler M (2009b): Characteristics of out-of-hospital paediatric emergencies attended by ambulance- and helicopter-based emergency physicians. Resuscitation $\underline{80}(8), 888-892$

Eich C, Roessler M, Nemeth M, Russo SG, Heuer JF, Timmermann A (2010): Reply to Letter: Paediatric tracheal prehospital intubation - What makes different our practice across the Ocean? Resuscitation $\underline{81}(5), 634-635$

El-Ganzouri AR, McCarthy RJ, Tuman KJ, Tanck EN, Ivankovich AD (1996): Preoperative

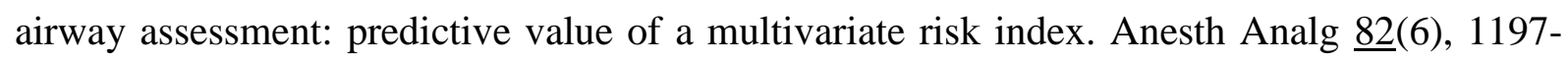
1204

Engdahl J, Axelsson A, Bang A, Karlson BW, Herlitz J (2003): The epidemiology of cardiac arrest in children and young adults. Resuscitation $\underline{58}(2), 131-138$

Eppich WJ, Adler MD, McGaghie WC (2006): Emergency and critical care pediatrics: use of medical simulation for training in acute pediatric emergencies. Curr Opin Pediatr 18(3), 266271

Firsching R, Messing-Jünger M, Rickels E, Gräber S, Schwerdtfeger K. (1996): Leitlinien der deutschen Gesellschaft für Neurochirurgie: Schädel-Hirn-Trauma im Erwachsenenalter. AWMF online. 
Fiser DH (1992): Assessing the outcome of pediatric intensive care. J Pediatr 121(1), 68-74

Fiser DH, Long N, Roberson PK, Hefley G, Zolten K, Brodie-Fowler M (2000): Relationship of pediatric overall performance category and pediatric cerebral performance category scores at pediatric intensive care unit discharge with outcome measures collected at hospital discharge and 1- and 6-month follow-up assessments. Crit Care Med 28(7), 2616-2620

Frei FJ, Erb T, Jonmarker C, Sümpelmann R, Werner O: Kinderanästhesie. 3. Auflage; Springer Medizin Verlag, Heidelberg 2004

Gausche M, Lewis RJ, Stratton SJ, Haynes BE, Gunter CS, Goodrich SM, Poore PD, McCollough MD, Henderson DP, Pratt FD, Seidel JS (2000): Effect of out-of-hospital pediatric endotracheal intubation on survival and neurological outcome: a controlled clinical trial. JAMA $\underline{283}(6), 783-790$

Gausche-Hill M, Lewis RJ, Gunter CS, Henderson DP, Haynes BE, Stratton SJ (2000): Design and implementation of a controlled trial of pediatric endotracheal intubation in the out-of-hospital setting. Ann Emerg Med 36(4), 356-365

Genzwuerker HV, Fritz A, Hinkelbein J, Finteis T, Schlaefer A, Schaeffer M, Thil E, Rapp HJ (2006): Prospective, randomized comparison of laryngeal tube and laryngeal mask airway in pediatric patients. Paediatr Anaesth $\underline{16}(12), 1251-1256$

Gerritse BM, Draaisma JM, Schalkwijk A, van Grunsven PM, Scheffer GJ (2008): Should EMS-paramedics perform paediatric tracheal intubation in the field? Resuscitation 79(2), 225229

Glaeser P (2000): Out-of-hospital intubation of children. JAMA 283(6), 797-798

Göttinger Statistisches Informationssystem (2008): Göttinger Umland: Landkreise in der Region Südniedersachsen, Nordhessen und Nordthüringen - Bevölkerung nach Altersgruppe und Geschlecht sowie Gebietsfläche und Bevölkerungsdichte 2004 bis 2007. http://www.goesis.goettingen.de 
Gries A, Zink W, Bernhard M, Messelken M, Schlechtriemen T (2006): Realistic assessment of the physician-staffed emergency services in Germany. Anaesthesist 55(10), 1080-1086

Guyette FX, Roth KR, LaCovey DC, Rittenberger JC (2007): Feasibility of laryngeal mask airway use by prehospital personnel in simulated pediatric respiratory arrest. Prehosp Emerg Care 11(2), 245-249

Handley AJ, Koster R, Monsieurs K, Perkins GD, Davies S, Bossaert L (2005): European Resuscitation Council guidelines for resuscitation 2005. Section 2. Adult basic life support and use of automated external defibrillators. Resuscitation 67 Suppl 1, 7-23

Hardman JG, Wills JS (2006): The development of hypoxaemia during apnoea in children: a computational modelling investigation. Br J Anaesth 97(4), 564-570

Harrison TH, Thomas SH, Wedel SK (2004): Success rates of pediatric intubation by a nonphysician-staffed critical care transport service. Pediatr Emerg Care 20(2), 101-107

Helm M, Hauke J, Frey W, Lampl L (1999): Der pädiatrische Traumapatient im Luftrettungsdienst. Notfall Rettungsmed 2, 150-157

Helm M, Hossfeld B, Schafer S, Hoitz J, Lampl L (2006): Factors influencing emergency intubation in the pre-hospital setting - a multicentre study in the German Helicopter Emergency Medical Service. Br J Anaesth 96(1), 67-71

Herlitz J, Engdahl J, Svensson L, Young M, Angquist KA, Holmberg S (2005): Characteristics and outcome among children suffering from out of hospital cardiac arrest in Sweden. Resuscitation 64(1), 37-40

Hoffmann F, Nicolai T (2009): Algorithmus zum Vorgehen bei häufigen respiratorischen Notfällen im Kindesalter. Notfall Rettungsmed $\underline{8}(12), 576-582$

Holm-Knudsen RJ, Rasmussen LS (2009): Paediatric airway management: basic aspects. Acta Anaesthesiol Scand 53(1), 1-9 
Holzki J, Laschat M, Puder C (2009): Iatrogenic damage to the pediatric airway. Mechanisms and scar development. Paediatr Anaesth $\underline{19}$ Suppl 1, 131-146

Hussain LM, Redmond AD (1994): Are pre-hospital deaths from accidental injury preventable? BMJ $\underline{308}, 1077-1080$

ILCOR (2000): Part 10: Pediatric advanced life support. European Resuscitation Council. Resuscitation 46 (1-3), 343-399

ILCOR (2005): Paediatric basic and advanced life support. Resuscitation 67(2-3), 271-303

Jennett B, Bond M (1975): Assessment of outcome after severe brain damage. Lancet 1975 , $1,480-484$

Jöhr M: Kinderanästhesie. 7. Auflage; Urban \& Fischer Verlag, München 2009

Katz SH, Falk JL (2001): Misplaced endotracheal tubes by paramedics in an urban emergency medical services system. Ann Emerg Med 37(1), 32-37

Kemp AM, Sibert JR (1991): Outcome in children who nearly drown: a British Isles study. BMJ $\underline{302}, 931-933$

Konrad C, Schupfer G, Wietlisbach M, Gerber H (1998): Learning manual skills in anesthesiology: is there a recommended number of cases for anesthetic procedures? Anesth Analg $\underline{86}(3), 635-639$

Kramer-Johansen J, Wik L, Steen PA (2006): Advanced cardiac life support before and after tracheal intubation - direct measurements of quality. Resuscitation 68(1), 61-69

Kuisma M, Suominen P, Korpela R (1995): Paediatric out-of-hospital cardiac arrests epidemiology and outcome. Resuscitation $\underline{30}(2), 141-150$

Langeron O, Masso E, Huraux C, Guggiari M, Bianchi A, Coriat P, Riou B (2000): Prediction of difficult mask ventilation. Anesthesiology 92(5), 1229-1236 
Lecky F, Bryden D, Little R, Tong N, Moulton C (2008): Emergency intubation for acutely ill and injured patients. Cochrane Database Syst Rev (2), CD001429

Lopez-Gil M, Brimacombe J, Alvarez M (1996): Safety and efficacy of the laryngeal mask airway. A prospective survey of 1400 children. Anaesthesia 51(10), 969-972

Mackay CA, Terris J, Coats TJ (2001): Prehospital rapid sequence induction by emergency physicians: is it safe? Emerg Med J $\underline{18}(1), 20-24$

Macnair D, Baraclough D, Wilson G, Bloch M, Engelhardt T (2009): Pediatric airway management: comparing the Berci-Kaplan Video Laryngoscope with direct laryngoscopy. Paediatr Anaesth 19 (6), 577-580

Maharaj CH, Costello JF, Harte BH, Laffey JG (2008): Evaluation of the Airtraq and Macintosh laryngoscopes in patients at increased risk for difficult tracheal intubation. Anaesthesia $\underline{63}(2), 182-188$

Martinon C, Duracher C, Blanot S, Perie-Vintras AC, Carli P, Meyer PG (2010): Paediatric prehospital tracheal intubation: What makes different our practice across the Ocean? Resuscitation 81(5), 634

Merkenschlager (2009): Der neurologische Notfall beim Kind. Notfall Rettungsmed 12(8), 590-599

Meyburg J, Bernhard M, Hoffmann GF, Motsch J (2009): Grundlagen für die Behandlung von Notfällen im Kindesalter. Dtsch Arztebl Int 106(45), 739-748

Meyer G, Orliaguet G, Blanot S, Jarreau MM, Charron B, Sauverzac R, Carli P (2000): Complications of emergency tracheal intubation in severely head-injured children. Paediatr Anaesth $\underline{10}(3), 253-260$

Moecke H, Dirks B, Friedrich HJ, Hennes HJ, Lackner CK, Messelken M, Neumann C, Pajonk FG, Reng M, Schachinger U, Violka T (2000): DIVI-Notarzteinsatzprotokoll, Version 4.0. Anaesthesist $\underline{49}$ (3), 211-213 
Mort TC (2004): Emergency tracheal intubation: complications associated with repeated laryngoscopic attempts. Anesth Analg 99(2), 607-613

Mort TC (2007a): Complications of emergency tracheal intubation: immediate airway-related consequences - part II. J Intensive Care Med 22(4), 208-215

Mort TC (2007b): Complications of emergency tracheal intubation: hemodynamic alterations - part I. J Intensive Care Med 22(3), 157-165

Nadkarni VM, Larkin GL, Peberdy MA, Carey SM, Kaye W, Mancini ME, Nichol G, LaneTruitt T, Potts J, Ornato JP, Berg RA (2006): First documented rhythm and clinical outcome from in-hospital cardiac arrest among children and adults. JAMA 295(1), 50-57

Neubert EM: Atemwegsmanagement in der Notfallmedizin. Evaluation des adäquaten Atemzugsvolumens bei Raumluftbeatmung des ungesicherten Luftweges. Med. Diss. Lübeck 2007

Newgard CD, Cudnik M, Warden CR, Hedges JR (2007): The predictive value and appropriate ranges of prehospital physiological parameters for high-risk injured children. Pediatr Emerg Care 23(7), 450-456

Nicolai T (2008): Pediatric resuscitation. Curr Opin Anaesthesiol 21(2), 204-208

Nicolai T, Hoffmann F (2009): Neue Techniken und Entwicklungen für die Notfallversorgung von Kindern. Notfall Rettungsmed 12(8), 600-606

Nolan JP, Soar J (2008): Airway techniques and ventilation strategies. Curr Opin Crit Care $\underline{14}(3), 279-286$

Paal P, Herff H, Mitterlechner T, von Goedecke A, Brugger H, Lindner KH, Wenzel V (2010a): Anaesthesia in prehospital emergencies and in the emergency room. Resuscitation 81(2), 148-154 
Paal P, Niederklapfer T, Keller C, von Goedecke A, Luckner G, Pehboeck D, Mitterlechner T, Herff H, Riccabona U, Wenzel V (2010b): Head-position angles in children for opening the upper airway. Resuscitation im Druck

Phillips BM, Mackway-Jones K, Jewkes F (2000): The European Resuscitation Council's paediatric life support course 'Advanced Paediatric Life Support'. Resuscitation 47(3), 329334

Pointer JE (1989): Clinical characteristics of paramedics' performance of pediatric endotracheal intubation. Am J Emerg Med $\underline{7}(4), 364-366$

Quan L, Kinder D (1992): Pediatric submersions: prehospital predictors of outcome. Pediatrics $\underline{90}(6), 909-913$

Quan L, Wentz KR, Gore EJ, Copass MK (1990): Outcome and predictors of outcome in pediatric submersion victims receiving prehospital care in King County, Washington. Pediatrics $\underline{86}(4), 586-593$

Redel A, Karademir F, Schlitterlau A, Frommer M, Scholtz LU, Kranke P, Kehl F, Roewer N, Lange M (2009): Validation of the GlideScope video laryngoscope in pediatric patients. Paediatr Anaesth 19(7), 667-671

Richard J, Osmond MH, Nesbitt L, Stiell IG (2006): Management and outcomes of pediatric patients transported by emergency medical services in a Canadian prehospital system. CJEM $\underline{8}(1), 6-12$

Roessler M, Zuzan O (2006): EMS systems in Germany. Resuscitation 68(1), 45-49

Russo SG, Eich C, Barwing J, Nickel EA, Braun U, Graf BM, Timmermann A (2007): Selfreported changes in attitude and behavior after attending a simulation-aided airway management course. J Clin Anesth 19(7), 517-522

Samsoon GL, Young JR (1987): Difficult tracheal intubation: a retrospective study. Anaesthesia $\underline{42}(5), 487-490$ 
Scheller B, Schalk R, Byhahn C, Peter N, L'Allemand N, Kessler P, Meininger D (2009): Laryngeal tube suction II for difficult airway management in neonates and small infants. Resuscitation $\underline{80}(7), 805-810$

Schlechtriemen T, Burghofer K, Lackner CK, Altemeyer KH (2005): Validierung des NACA-Score anhand objektivierbarer Parameter: Untersuchung an 104.962 Primäreinsätzen der Jahre 1999-2003 aus der Luftrettung. Notfall Rettungsmed $\underline{8}$, 96-108

Schlechtriemen T, Masson R, Burghofer K, Lackner CK, Altemeyer KH (2006): Pädiatrische Notfälle in der präklinischen Notfallmedizin: Schwerpunkte des Einsatzspektrums im bodengebundenen Rettungsdienst und in der Luftrettung. Anaesthesist 55(3), 255-262

Schmid MC, Deisenberg M, Strauss H, Schuttler J, Birkholz T (2006): Ausstattung bodengebundener Notarztrettungsmittel in Bayern: Eine Umfrage. Anaesthesist 55(10), 10511057

Schmid MC, Mang H, Ey K, Braun J, Schuttler J (2009): Atemwegsmanagement im deutschen Luftrettungsdienst. Anaesthesist $\underline{58(9), ~ 884-890 ~}$

Schmidt U, Geerling J, Fuhler M, Hubrich V, Richter M, Krettek C (2002): Die präklinische Versorgung des pädiatrischen Traumapatienten: Ein retrospektiver Vergleich zwischen luftund bodengebundener Rettung. Unfallchirurg 105(11), 1000-1006

Scott DB (1986): Endotracheal intubation: friend or foe. Br Med J (Clin Res Ed) 292(6514), $157-158$

Sexton JB, Thomas EJ, Helmreich RL (2000): Fehlverhalten, Überbelastung und Teamwork in Medizin und Luftfahrt. Eine fachübergreifende Studie. Chirurg 71(6 Suppl 1), 138-142

Silvestri S, Ralls GA, Krauss B, Thundiyil J, Rothrock SG, Senn A, Carter E, Falk J (2005): The effectiveness of out-of-hospital use of continuous end-tidal carbon dioxide monitoring on the rate of unrecognized misplaced intubation within a regional emergency medical services system. Ann Emerg Med 45(5), 497-503 
Simpson DA, Cockington RA, Hanieh A, Raftos J, Reilly PL (1991): Head injuries in infants and young children: the value of the Paediatric Coma Scale. Review of literature and report on a study. Childs Nerv Syst $\underline{7}(4), 183-190$

Sirbaugh PE, Pepe PE, Shook JE, Kimball KT, Goldman MJ, Ward MA, Mann DM (1999): A prospective, population-based study of the demographics, epidemiology, management, and outcome of out-of-hospital pediatric cardiopulmonary arrest. Ann Emerg Med 33(2), 174-184

Statistisches Bundesamt Wiesbaden: Todesursachen in Deutschland. Fachserie 12, Reihe 4; Metzler-Poerschel, Stuttgart 2000

Stein SC, Spettell CM (1995): Delayed and progressive brain injury in children and adolescents with head trauma. Pediatr Neurosurg 23(6), 299-304

Stenke CP: Der Münchener Kindernotarzt. 3667 Kindernotarzteinsätze der Jahre 1998 - 2001 untersucht an zwei der vier beteiligten Kliniken. Med. Diss. München 2004

Stockinger ZT, McSwain NE (2004): Prehospital endotracheal intubation for trauma does not improve survival over bag-valve-mask ventilation. J Trauma $\underline{56}(3), 531-536$

Strauss J, Becke K (2010): Auf bekanntes Material zurückgreifen. Dtsch Arzteb Int 2010 $\underline{107}(17), 304$

Suominen P, Silfvast T, Korpela R, Erosuo J (1996): Pediatric prehospital care provided by a physician-staffed emergency medical helicopter unit in Finland. Pediatr Emerg Care 12(3), $169-172$

Suominen P, Korpela R, Kuisma M, Silfvast T, Olkkola KT (1997): Paediatric cardiac arrest and resuscitation provided by physician-staffed emergency care units. Acta Anaesthesiol Scand $\underline{41}(2), 260-265$

Suominen P, Baillie C, Kivioja A, Ohman J, Olkkola KT (2000a): Intubation and survival in severe paediatric blunt head injury. Eur J Emerg Med 7(1), 3-7 
Suominen P, Olkkola KT, Voipio V, Korpela R, Palo R, Rasanen J (2000b): Utstein style reporting of in-hospital paediatric cardiopulmonary resuscitation. Resuscitation $\underline{45}(1), 17-25$

Suominen P, Baillie C, Korpela R, Rautanen S, Ranta S, Olkkola KT (2002): Impact of age, submersion time and water temperature on outcome in near-drowning. Resuscitation $\underline{52}(3)$, $247-254$

Teasdale G, Jennett B (1974): Assessment of coma and impaired consciousness. A practical scale. Lancet $\underline{1974,2}, 81-84$

Timmermann A, Russo SG (2007): Which airway should I use? Curr Opin Anaesthesiol $\underline{20}(6), 595-599$

Timmermann A, Eich C, Russo SG, Natge U, Brauer A, Rosenblatt WH, Braun U (2006): Prehospital airway management: a prospective evaluation of anaesthesia trained emergency physicians. Resuscitation $\underline{70}(2), 179-185$

Timmermann A, Russo SG, Rosenblatt WH, Eich C, Barwing J, Roessler M, Graf BM (2007a): Intubating laryngeal mask airway for difficult out-of-hospital airway management: a prospective evaluation. Br J Anaesth $\underline{99}(2), 286-291$

Timmermann A, Braun U, Panzer W, Schlaeger M, Schnitzker M, Graf BM (2007b): Präklinisches Atemwegsmanagement in Norddeutschland. Individuelle Kenntnisse, Vorgehen und Ausrüstung. Anaesthesist 56(4), 328-334

Timmermann A, Russo SG, Eich C, Roessler M, Braun U, Rosenblatt WH, Quintel M (2007c): The out-of-hospital esophageal and endobronchial intubations performed by emergency physicians. Anesth Analg 104(3), 619-623

Timmermann A, Russo SG, Hollmann MW (2008): Paramedic versus emergency physician emergency medical service: role of the anaesthesiologist and the European versus the AngloAmerican concept. Curr Opin Anaesthesiol 21(2), 222-227 
Topjian AA, Berg RA, Nadkarni VM (2008): Pediatric cardiopulmonary resuscitation: advances in science, techniques, and outcomes. Pediatrics 122(5), 1086-1098

Trevisanuto D, Fornaro E, Verghese C (2006): The GlideScope video laryngoscope: initial experience in five neonates. Can J Anaesth 53(4), 423-424

Tryba M, Brüggemann H, Echtermeyer V (1980): Klassifizierung von Erkrankungen und Verletzungen in Notarztrettungssystemen. Notfallmedizin $\underline{6}, 725-727$

Ummenhofer W, Scheidegger D (2002): Role of the physician in prehospital management of trauma: European perspective. Curr Opin Crit Care $\underline{8}(6), 559-565$

Vlatten A, Aucoin S, Litz S, Macmanus B, Soder C (2009): A comparison of the STORZ video laryngoscope and standard direct laryngoscopy for intubation in the Pediatric airway - a randomized clinical trial. Paediatr Anaesth 19(11), 1102-1107

Von Elm E, Schoettker P, Henzi I, Osterwalder J, Walder B (2009): Pre-hospital tracheal intubation in patients with traumatic brain injury: systematic review of current evidence. $\mathrm{Br} \mathrm{J}$ Anaesth 103(3), 371-386

Von Goedecke A, Keller C, Voelckel WG, Dunser M, Paal P, Torgersen C, Wenzel V (2006): Maskenbeatmung als Rückzugsstrategie zur endotrachealen Intubation. Anaesthesist $\underline{55}(1), 70-79$

Von Goedecke A, Herff H, Paal P, Dorges V, Wenzel V (2007): Field airway management disasters. Anesth Analg 104(3), 481-483

Wagner B (2009): Schweres Schädel-Hirn-Traum. Monitoring und Mangagement beim Kind. Notfall Rettungsmed 12(2), 133-138

Walker RW (2001): Management of the difficult airway in children. J R Soc Med 94(7), 341344 
Walker RW, Ellwood J (2009): The management of difficult intubation in children. Paediatr Anaesth $\underline{19}$ Suppl 1, 77-87

Wang HE, Yealy DM (2006a): Out-of-hospital endotracheal intubation: where are we? Ann Emerg Med 477(6), 532-541

Wang HE, Yealy DM (2006b): How many attempts are required to accomplish out-ofhospital endotracheal intubation? Acad Emerg Med 13(4), 372-377

Wang HE, Cook LJ, Chang CC, Yealy DM, Lave JR (2009a): Outcomes after out-of-hospital endotracheal intubation errors. Resuscitation $\underline{80}(1), 50-55$

Wang HE, Simeone SJ, Weaver MD, Callaway CW (2009b): Interruptions in cardiopulmonary resuscitation from paramedic endotracheal intubation. Ann Emerg Med $\underline{54}(5), 645-652$

Weiss M, Bernoulli L, Zollinger A (2001a): Der NACA-Index. Aussagekraft und Stellenwert des modifizierten NACA-Indexes in der präklinischen Schweregraderfassung von Unfallpatienten. Anaesthesist 50(3), 150-154

Weiss M, Schwarz U, Dillier C, Fischer J, Gerber AC (2001b): Use of the intubating laryngeal mask in children: an evaluation using video-endoscopic monitoring. Eur $\mathbf{J}$ Anaesthesiol 18(11), 739-744

Weiss M, Mauch J, Becke K, Schmidt J, Joehr M (2009): Fiberoptisch unterstützte endotracheale Intubation durch die Larynxmaske im Kindesalter. Anaesthesist 58(7), 716-721

Wiese CH, Bahr J, Bergmann A, Bergmann I, Bartels U, Graf BM (2008): "No-flow-time"Reduzierung durch Einsatz des Larynxtubus: Vergleich zur Beutel-Masken-Beatmung. Anaesthesist 57(6), 589-596

Wiese CH, Semmel T, Mueller JU, Bahr J, Ocker H, Graf BM (2009): The use of the laryngeal tube disposable (LT-D) by paramedics during out-of-hospital resuscitation-an observational study concerning ERC guidelines 2005. Resuscitation 80 (2), 194-198 
Wu ET, Li MJ, Huang SC, Wang CC, Liu YP, Lu FL, Ko WJ, Wang MJ, Wang JK, Wu MH (2009): Survey of outcome of CPR in pediatric in-hospital cardiac arrest in a medical center in Taiwan. Resuscitation 80(4), 443-448

Young KD, Gausche-Hill M, McClung CD, Lewis RJ (2004): A prospective, populationbased study of the epidemiology and outcome of out-of-hospital pediatric cardiopulmonary arrest. Pediatrics $\underline{114}(1), 157-164$

Youngquist S, Gausche-Hill M, Burbulys D (2007): Alternative airway devices for use in children requiring prehospital airway management: update and case discussion. Pediatr Emerg Care $\underline{23}(4), 250-258$

Zaritsky A, Nadkarni V, Hazinski MF, Foltin G, Quan L, Wright J, Fiser D, Zideman D, O'Malley P, Chameides L (1995): Recommended guidelines for uniform reporting of pediatric advanced life support: the Pediatric Utstein Style. A statement for healthcare professionals from a task force of the American Academy of Pediatrics, the American Heart Association, and the European Resuscitation Council. Resuscitation 30(2), 95-115 


\section{Danksagung}

Ich bedanke mich bei meinem Doktorvater Herrn Prof. Dr. med. M. Quintel, Direktor der Abteilung Anaesthesiologie im Zentrum Anaesthesiologie, Rettungs- und Intensivmedizin (ZARI), für die freundliche Überlassung des Themas.

Mein Dank gilt ferner den Notärzten des ZARI für ihre gewissenhafte Dokumentation der Einsätze sowie Herrn Meinert Puttendörfer ( † für seine wertvolle Unterstützung bei der Auswertung der RED-Daten.

Zu besonderem Dank bin ich meinem Betreuer Herrn Priv.-Doz. Dr. med. Christoph Bernhard Eich (ZARI) verpflichtet. Durch seine Motivation, seine stets hilfreiche Unterstuitzung und seine kritische Durchsicht des Manuskripts hat er maßgeblich zum Gelingen dieser Dissertation beigetragen. 